\title{
Correlation Effects in Kinetics of One-Dimensional Atomic Systems
}

\author{
V. D. Borman, I. V. Tronin, V. N. Tronin, \\ V. I. Troyan, and O. S. Vasiliev \\ National Research Nuclear University, MEPhI, Kashirskoye Sh. 31, Moscow 115409, Russia \\ Correspondence should be addressed to V. D. Borman; vdborman@mephi.ru
}

Received 10 January 2013; Accepted 7 March 2013

Academic Editor: Yun Zhao

Copyright @ 2013 V. D. Borman et al. This is an open access article distributed under the Creative Commons Attribution License, which permits unrestricted use, distribution, and reproduction in any medium, provided the original work is properly cited.

\begin{abstract}
The paper is devoted to the analysis of the correlation effects and manifestations of general properties of 1D systems (such as spatial heterogeneity that is associated with strong density fluctuations, the lack of phase transitions, the presence of frozen disorder, confinement, and blocked movement of nuclear particle by its neighbours) in nonequilibrium phenomena by considering the four examples. The anomalous transport in zeolite channels is considered. The mechanism of the transport may appear in carbon nanotubes and MOF structures, relaxation, mechanical properties, and stability of nonequilibrium states of free chains of metal atoms, non-Einstein atomic mobility in $1 \mathrm{D}$ atomic systems. Also we discuss atomic transport and separation of two-component mixture of atoms in a 1D system - a zeolite membrane with subnanometer channels. We discuss the atomic transport and separation of two-component mixture of atoms in a 1D system-zeolite membrane with subnanometer channels. These phenomena are described by the response function method for nonequilibrium systems of arbitrary density that allows us to calculate the dynamic response function and the spectrum of relaxation of density fluctuations $1 \mathrm{D}$ atomic system.
\end{abstract}

\section{Introduction}

In recent years, one-dimensional objects became available and actively investigated due to their unique properties. They are free chains of metals just one atom thick $(\mathrm{Au}, \mathrm{Ag}, \mathrm{Pt}$, In, $\mathrm{Mn}, \mathrm{Li}$, and $\mathrm{Ni}$ ) [1-13], chains on specially prepared surfaces of crystals with high crystalline indices [14-19], the molecules (atoms) in subnanometer channels of complex oxides, zeolites [20-22], carbon nanotubes [23-27], and in some metal organic frameworks (MOF).

According to modern concepts, the common feature of one-dimensional objects as statistical systems is spatially inhomogeneous state and density fluctuations. These fluctuations are caused by the crucial role of many-particle correlations in $1 \mathrm{D}$ systems. This feature is connected with the absence of phase transitions in $1 \mathrm{D}$ systems of particles of one kind with short-range potential of the interparticle interaction (see van Hove Theorem [28, 29]). In this case, for certain values of the density the susceptibility of $1 \mathrm{D}$ system has a maximum but stays finite in contrast to 3D systems, where susceptibility becomes infinite in vapour-liquid and liquid-solid phase transitions [30].

The absence of phase transition means that stable nuclei of a new phase are not formed, and the one-dimensional clusters nuclei that are formed in a system have a finite lifetime and are the density fluctuations. 1D systems are characterized by frozen disorder because the atomic particles cannot be exchanged with their neighbours and block the movement of each particle neighbors. For kinetic phenomena in atomic $1 \mathrm{D}$ confinement system, the role of confinement is not only to preserve the geometry of the system, but also the system relaxation by phonons. Relaxation of the system in confinement due to strong interparticle correlations depends on the ratio of the energy of particle interaction with confinement and interparticle interaction [31].

This paper is devoted to the analysis of correlation effects and manifestations of these general properties of nonequilibrium phenomena in the four examples. The anomalous transport in zeolite channels is considered. The mechanism 
of the transport may appear in carbon nanotubes and MOF structures, relaxation, mechanical properties, and stability of nonequilibrium states of free chains of metal atoms, nonEinstein atomic mobility in $1 \mathrm{D}$ atomic systems. Also we discuss atomic transport and separation of two-component mixture of atoms in a $1 \mathrm{D}$ system-a zeolite membrane with subnanometer channels.

These phenomena are described below using the response function method for nonequilibrium systems of arbitrary density [31]. This method allows us to calculate the dynamic response function and the spectrum of relaxation of density fluctuations of $1 \mathrm{D}$ atomic system. The spectrum contains diffusion and hydrodynamic modes. In the latter case, the relaxation of the system and the nuclear transport occurs through the distribution of density waves and not by the diffusion of particles. This relaxation mechanism becomes the determining by increasing the density that allows one to explain the abnormally high rate of atomic transport in subnanometer channels. The change of relaxation mechanism by increase of the density is associated with the observed non-Einstein character of mobility of atomic particles (the law for the mean square displacement $x^{2}=2 D t$ is violated ( $D$ is the diffusion coefficient, and $t$ is the time of observation) [32]). The found spectrum of relaxation allows to calculate the probability of decay of 1D free clusters of various lengths [30] and to explain the dependence of the stability of clusters of metal atoms on the chain atoms properties, the mechanical state of a cluster under the influence of an external force, and the number of particles in a stable cluster. These values were measured simultaneously in experiments [13].

The response function method was used to describe the practically important transport of two-component mixture in membranes with subnanometer channels.

It was shown that a phase transition in an inhomogeneous state may occur in $1 \mathrm{D}$ system of particles of two kinds with the increase of filling factor. Such a phase transition occurs due to an effective particle attraction and is characterised by the formation of clusters of a finite size [33]. The amplitude of the fluctuations in the density corresponding to the formation of such a state is the order parameter. An equation for the order parameter for a mixture of particles in the channels is derived. In contrast to the well-known phenomenological LandauGinzburg equation [34], the coefficients of the equation are calculated for a known interparticle interaction potential depending on the density and temperature. The analysis of this equation allows us to describe the observed increase in the separation factor with increasing flux through $1 \mathrm{D}$ channels at their closing by a stable clusters with a certain concentration of the components and determine the dependence of the separation on the concentration, temperature, and density.

\section{Materials and Methods}

2.1. Molecular Transport in Subnanometer Channels. Molecular transport in nanometer and subnanometer channels in porous bodies is currently drawing a great deal of interest from the standpoint of fundamental science [35-39] and because of the many applications of membrane and nanotechnologies in various fields ranging from nuclear power to ecology [40,41]. Polycrystalline ceramic membranes consisting of complex oxides (zeolites), possessing subnanometer channels ranging in diameter from 0.3 to $1.4 \mathrm{~nm}$, have been synthesized recently [40]. On account of the high selectivity of molecular transport in zeolite membranes, as compared with the wellknown polymeric membranes [41-43], new technologies for separation, reprocessing, and utilization of materials are being intensively developed on the basis of zeolite membranes [41-43].

When the channel diameters in membranes decrease to the nanometer scale, molecular transport is determined by the Knudsen flow in the central zone of the channel, free of interaction of molecules with walls, and particle diffusion in the surface force field [39]. In subnanometer channels, the interaction potentials between molecules and the opposite walls overlap, and molecular transport occurs under conditions of a constant interaction of molecules with a solid. Consequently, the diffusion coefficient in the limit of small filling numbers of a channel is determined by the relaxation of particles on phonons and surface defects [37]. For molecules, with the exception of light particles $(H$, $\mathrm{He})$, the channel walls are impenetrable, and consequently molecular transport is possible only along the channel axes. In this sense, it is different from diffusion in solids and may be assumed to be one-dimensional. In contrast to surface diffusion in channels with diameter $d>1 \mathrm{~nm}$, a fundamentally new property of molecular transport should appear in subnanometer channels. As the filling factor of a channel or the diameter $(\sigma)$ of molecules increases, so that $d<2 \sigma$, each molecule can block the motion of two molecules. Consequently, it can be expected that as the diameter of the molecules or the external gas pressure increases, the molecular flux in membranes with subnanometer channels should decrease. However, experiments have shown that the diffusion flux of a number of molecules $\left(\mathrm{CH}_{4}, \mathrm{C}_{2} \mathrm{H}_{6}\right.$, and others) in a ZSM-5 zeolite membrane with channel diameters $0.54-0.57 \mathrm{~nm}$ does not decrease but rather increases as the external gas pressure increases [43]. It has also been established that the diffusion coefficient for these gases increases by more than an order of magnitude as the filling factor of the channels with molecules increases. It has been found that for a number of gases, the temperature dependence of the flux possesses a maximum and a minimum.

In this section, the transport of a single-component molecular gas (system of atomic particles) in subnanometerdiameter channels is investigated theoretically.

2.1.1. Sorption Isotherm in a $1 D$ Channel. Let us consider a surface in contact with an ideal single-component gas with temperature $T$ and pressure $p$. Let us assume that particles located on the surface do not interact with one another. We will also assume that the energy of the gas molecules on the surface is $\varepsilon_{0}$. Let there be $N$ adsorption centers on the surface. Then the average number $\bar{N}_{1}$ of particles adsorbed on 
the surface as a function of the gas pressure and temperature has the form (Langmuir isotherm)

$$
\begin{aligned}
& \frac{\bar{N}_{1}}{N}=\frac{p}{p+p_{0}(T)} \\
& p_{0}(T) \equiv T\left(\frac{\hbar^{2}}{2 \pi m T}\right)^{-3 / 2} \exp \left(-\beta \varepsilon_{0}\right),
\end{aligned}
$$

where $m$ is the mass of a gas molecule, $\hbar$ is Planck's constant, and $\beta=T^{-1}$ is the reciprocal of the temperature. The method used to derive the Langmuir isotherms (1) admits wide extensions. Specifically, this method can be used to solve the problem of the filling factor of a cylindrical channel with diameter $d$ taking into account the interparticle interaction in the channel. Let the channel diameter be comparable to the maximum diameter of a gas molecule. Let us consider the equilibrium of the gas with the surface on which $k$ of the channels described previously emerge. Let $\varepsilon_{1}$ be the binding energy of a particle at the entrance to the channel. If the energy $\varepsilon_{1}$ is negative, then it is energetically favorable for the gas molecule to enter the channel. Let $q$ be the total number of particles in a channel of length $L, n$ the total number of particles in the channel and on the surface, and $N_{0}$ the number of settling locations in the channel. Then the partition function for the grand canonical ensemble, taking into account the interaction of the gas particles in the channel, is

$$
\begin{gathered}
\Theta=\sum \frac{(N-k) ! \exp \left[\beta \varepsilon_{0}\left(N_{1}-n\right)\right]}{\left(N_{1}-n\right) !\left(N-k-\left(N_{1}-n\right)\right) !} \\
\quad \times \frac{k ! \exp \left[\beta \varepsilon_{1}(n-q)\right]}{(n-q) !(k-(n-q)) !} \\
\quad \times \frac{N_{0} ! \exp \left(\beta \varepsilon_{2} q\right)}{q !\left(N_{0}-q\right) !} \exp \left(\beta \mu N_{1}\right) Z_{\text {int }}(q), \\
N_{1}+q=n .
\end{gathered}
$$

Here $\varepsilon_{2}$ is the binding energy of the particles in the channel and $Z_{\text {int }}(q)$ is the partition function, corresponding to taking account of the interaction of the gas particles in the channel. Since $Z_{\text {int }}$ depends on the number $q$ of particles in the channel, it is impossible to calculate the partition function (2) in the grand canonical ensemble in the general case. However, in the problem of filling of a channel, the states for which the number of particles in the channel $q \gg 1$ should make the main contribution to the partition function (2). Then the quantity $Z_{\text {int }}(q)$ can be replaced by the partition function of interacting particles in the channel, calculated with the average number $q$ of particles in the channel:

$$
Z_{\text {int }}(q) \simeq Z_{\text {int }}(\bar{q}) \text {. }
$$

The approximation (3) corresponds to the thermodynamic limit $(q \gg 1)$ for gas (system of atomic particles) molecules located in the channel. Using the relation (3), the partition function (2) can be easily calculated. Using the condition for equilibrium between the gas and the surface, we obtain for the average number $\bar{q}$ of particles in the channel

$$
\begin{gathered}
\frac{\bar{q}}{N_{0}}=\frac{p}{p+\tilde{p}(T, \bar{q})}, \quad \tilde{p}(\bar{q}, T)=\frac{\exp [-\beta \varepsilon(\bar{q}, T)]}{\alpha(T)}, \\
(\alpha(T))^{-1}=T\left(\frac{\hbar^{2}}{2 m \pi T}\right)^{-3 / 2}, \quad \varepsilon=\varepsilon_{1}-F_{\mathrm{int}}(\bar{q}, T), \\
F_{\mathrm{int}}(\bar{q}, T) \equiv-T \ln Z_{\mathrm{int}}(\bar{q}, T) .
\end{gathered}
$$

Here $F_{\text {int }}(\bar{q}, T)$ is the free energy of interaction per atomic particle in the channel. Instead of the number $N_{0}$ of settling locations in the channel, it is convenient to introduce the average distance between settling locations in the channel $\left(\eta=L / N_{0}\right)$ and to replace $\bar{q}$ by the filling factor $\theta \equiv \bar{q} \sigma / L$ of particles in the channel. Then, we obtain from (4) an equation determining the pressure and temperature dependences of the filling factor $\theta$ of the channel:

$$
\frac{\eta \theta}{\sigma}=\frac{p}{p+\widetilde{p}(T, \theta)} .
$$

The relations (4) show that the problem of obtaining the equation for the adsorption $\theta(p, T)$, determining the filling factor at various pressures and temperatures of the gas above the surface, reduces to calculating the quantity $F_{\text {int }}(\theta, T) \equiv$ $-T \ln Z_{\text {int }}(\theta, T)$, determining the "correction" to the pressure as a result of the interaction of the particles in the channel. Thus, to obtain the adsorption isotherms in the system under study, it is necessary to calculate the total free energy of the particles in a subnanometer channel taking into account their interaction with the channel wall and with one another. For this, we will consider $N$ particles in a channel whose size is comparable to the average particle diameter. In the general case, the total potential energy of such a system can be written as

$$
E\left(\mathbf{r}_{1}, \ldots, \mathbf{r}_{N}\right)=\sum_{i, j=1}^{N} V\left(\mathbf{r}_{i}-\mathbf{r}_{j}\right)+\sum_{i=1}^{N} U\left(\mathbf{r}_{i}\right)
$$

Here $V\left(\mathbf{r}_{i}-\mathbf{r}_{j}\right)$ is the potential energy of the pair interaction of the particles located at points with coordinates $\mathbf{r}_{i}$ and $\mathbf{r}_{j}$; $U\left(\mathbf{r}_{i}\right)$ is the interaction energy between a particle located at the point $\mathbf{r}_{i}$ and the channel walls. We will transform the expression for the pair interaction potential of the particles using the fact that the channel diameter is comparable to the particle diameter. Then the interaction potential depends only on the particle coordinates along the channel $r$. We write the expression for $V\left(\mathbf{r}_{i}-\mathbf{r}_{j}\right)$ in the form

$$
\begin{aligned}
V\left(\mathbf{r}_{i}-\mathbf{r}_{j}\right) & \equiv V\left(x_{i}-x_{j}, y_{i}-y_{j}, r_{i}-r_{j}\right) \\
& \simeq V\left(r_{i}-r_{j}, 0,0\right) \equiv \Phi\left(r_{i}-r_{j}\right) .
\end{aligned}
$$

This relation holds when the channel diameter is comparable to the particle diameter, and the pair interaction potential $V\left(\mathbf{r}_{i}-\mathbf{r}_{j}\right)$ of the particles is not a long-range potential. 
It is convenient to represent the interaction energy between a particle and the channel walls in the form

$$
U\left(\mathbf{r}_{i}\right)=U\left(\mathbf{r}_{i}^{\prime}, r_{i}\right)
$$

Here $\mathbf{r}_{i}^{\prime}$ is a dimensionless two-dimensional radius vector in a plane perpendicular to the channel. It is convenient to write the partition function of such a system in the form

$$
\begin{aligned}
Z_{N} \propto \int & d \mathbf{r}_{1}^{\prime} \cdots d \mathbf{r}_{N}^{\prime} d z_{1} \cdots d z_{N} \exp \left(-\beta U_{N}\right) \\
& \times \exp \left[-\beta \sum_{i=1}^{N} U\left(\mathbf{r}_{i}^{\prime}, r_{i}\right)\right] .
\end{aligned}
$$

Here

$$
U_{N}=\sum_{1 \leq i, j \leq N} \Phi\left(r_{i}-r_{j}\right)
$$

The integration over the particle coordinates in a plane perpendicular to the channel can be performed exactly:

$$
\begin{aligned}
& \int d \mathbf{r}_{1}^{\prime} \cdots d \mathbf{r}_{N}^{\prime} \exp \left[-\beta \sum_{i=1}^{N} U\left(\mathbf{r}_{i}^{\prime}, r_{i}\right)\right] \\
& =\int d \mathbf{r}_{1}^{\prime} \exp \left[-\beta U\left(\mathbf{r}_{1}^{\prime}, r_{1}\right)\right] \int d \mathbf{r}_{2}^{\prime} \exp \left[-\beta U\left(\mathbf{r}_{2}^{\prime}, r_{2}\right)\right] \\
& \quad \times \cdots \times \int d \mathbf{r}_{N}^{\prime} \exp \left[-\beta U\left(\mathbf{r}_{N}^{\prime}, r_{N}\right)\right] \\
& \equiv \exp \left[-\beta \sum_{i=1}^{N} \Psi\left(r_{i}\right)\right] .
\end{aligned}
$$

Here $\Psi\left(r_{i}\right)$ is the effective potential in which a particle moves in the channel as a result of the interaction of the particle with the walls:

$$
\exp [-\beta \Psi(r)] \equiv \int \exp \left[-\beta U\left(\mathbf{r}^{\prime}, r\right)\right] d \mathbf{r}^{\prime} .
$$

Let us assume that the energy of a gas molecule interacting with the channel surface is constant and equal to $\varepsilon_{1}=$ $\Psi\left(r_{\text {min }}\right)=\Psi(0)$. Then

$$
Z_{N}^{\prime} \propto \exp \left(-\beta N \varepsilon_{2}\right) \int d r_{1} \cdots d r_{N} \exp \left(-\beta U_{N}\right)
$$

The energy $\varepsilon_{1}$ physically corresponds to the binding energy of a particle in the surface potential $\Psi(r)$. Thus, under the assumptions made previously, the gas in the channel can be assumed to be one-dimensional (the channel diameter is comparable to the maximum diameter of an atomic particle). It is well known $[44,45]$ that the partition function of a one-dimensional gas with an arbitrary interaction potential can be calculated exactly under certain assumptions, which are formulated in the following. Indeed, let us consider an equilibrium system of $N$ particles in a channel which possesses only one degree of freedom per particle that are located in the segment $[0, L]$ of the $r$ axis. The total partition function of the system has the form

$$
\widetilde{Z}_{N}=\left(\frac{m T}{2 \pi \hbar^{2}}\right) \frac{Q_{N}}{N !} \exp \left(-\beta N \varepsilon_{1}\right),
$$

where

$$
\begin{gathered}
Q_{N}=\int_{0}^{L} \cdots \int_{0}^{L} \exp \left(-\beta U_{N}\right) d r_{1} \cdots d r_{N} \\
U_{N}=\sum_{1 \leq y \leq N-1} \Phi\left(r_{i+1}-r_{i}\right) .
\end{gathered}
$$

Here $\Phi(r)$ is the pair interaction potential of the particles in the channel. To calculate $F_{\text {int }}$, the cofactor responsible for the partition function of an ideal gas must be eliminated from the partition function (14). In our problem, the cofactor corresponding to a gas of particles in a channel without a pair interaction potential between the particles is already included in the expression (2). Thus, since the partition function of a one-dimensional gas without an interaction has the form

$$
Z_{\text {int }}=\left(\frac{m T}{2 \pi \hbar^{2}}\right)^{N / 2} \frac{L^{N}}{N !} \exp \left(-\beta N \varepsilon_{2}\right),
$$

we obtain from (2)

$$
F_{\text {int }}=-T \frac{\ln \widetilde{Z}_{\widetilde{N}}(\theta, T)}{Z_{i d}}=-T \ln \left(\frac{Q_{N}}{L^{N}}\right) .
$$

Thus, the problem has been reduced to calculating the configuration integral

$$
\begin{aligned}
Q_{N}=\int \cdots \int \exp [ & -\beta\left\{\Phi\left(\xi_{1}\right)+\Phi\left(\xi_{2}\right)\right. \\
& \left.\left.+\cdots+\Phi\left(\xi_{N}\right)\right\}\right] d \xi_{1} \cdots d \xi_{N}, \\
\xi_{i} & \equiv r_{i+1}-r_{i} .
\end{aligned}
$$

In studying the configuration space of a system of particles in a one-dimensional channel, it should be kept in mind that the quantities $\xi_{i}$ are not independent. They are related as

$$
\sum_{j=1}^{N} \xi_{j}=L
$$

where $L$ is the total length of the channel. Physically, this relation corresponds to the impossibility of particles penetrating one another and the condition of "blocking" of particles with diameter $\sigma$ in a channel whose diameter $d<2 \sigma$. An explicit expression for the configuration integral can be obtained if the explicit form of the pair interaction potential of the particles is known. We will consider a simple but nontrivial case: a system of hard spheres with diameter $\sigma$. The expression for the interatomic interaction energy in this case is

$$
\Phi_{i}(\xi)= \begin{cases}\infty & \text { for } \xi<\sigma \\ 0 & \text { for } \xi \geq \sigma\end{cases}
$$


We obtain for $F_{\text {int }}$ in the hard-sphere approximation

$$
F_{\text {int }}(\theta, T)=-T \ln (1-\theta) .
$$

Thus, in the hard-sphere approximation, the adsorption isotherm for a gas in a subnanometer channel has the form

$$
\begin{gathered}
\frac{\eta \theta}{\sigma}=\frac{(1-\theta) p}{(1-\theta) p+\widetilde{p}_{0}(T)}, \\
\widetilde{p}_{0}(T) \equiv T\left(\frac{\hbar^{2}}{2 \pi m T}\right)^{-3 / 2} \exp \left(-\beta \varepsilon_{1}\right) .
\end{gathered}
$$

The relation (22) makes it possible to construct the dependence of the filling factor on the gas pressure for various molecules. It should be kept in mind that the sign of the energy $\varepsilon_{1}$ determines the possibility or impossibility of a particle entering a channel: for $\varepsilon_{1}>0$, it is energetically favorable for a gas particle to enter the channel for any external pressure greater than $p_{0}$. For $\varepsilon_{1}<0$, the particles must overcome a potential barrier to enter the channel. The configuration integral can be calculated exactly for an arbitrary interaction between the particles in the channels [45]. This makes it possible to obtain an equation of state of a $1 \mathrm{D}$ gas for an arbitrary interaction. Thus, for an interparticle interaction potential of the form

$$
\Phi(z)= \begin{cases}+\infty & \text { for } r \leq \sigma \\ -\varepsilon & \text { for } \sigma<r \leq \sigma+R \\ 0 & \text { for } r<R+\sigma\end{cases}
$$

where $r$ is the effective radius of attraction, the equation of state of a one-dimensional gas is

$$
p_{1 \mathrm{D}} \sigma\left(\frac{1}{\theta}-1\right)=T-p_{1 \mathrm{D}} R\left[\frac{\exp \left(\beta p_{1 \mathrm{D}} R\right)}{1-\exp (-\beta \varepsilon)}-1\right]^{-1} \text {. }
$$

In the limits $\varepsilon \rightarrow 0$ and $R \rightarrow 0$, this equation becomes the equation of state of a system of hard spheres. The isotherms calculated numerically starting from (24) are presented in Figure 1. It is also evident in this figure that at high temperatures the gas behaves almost as an ideal gas $\left(p_{1 \mathrm{D}} \propto \theta\right)$. At low temperatures the isotherms seem to consist of two parts. For high density $\theta \simeq$ const, which is typical for a condensed phase, whereas at pressure less than a characteristic value a gas-like phase for which $p_{1 \mathrm{D}} \propto \theta$ appears.

It can be shown that at a transition from one regime to another, the free energy of the system has no singularities. For this reason, there is no exact analogy with phase transitions. This assertion agrees completely with the Landau-van Hove Theorem [44], according to which any one-dimensional model of a gas with a finite interaction radius does not undergo phase transitions. On the other hand, it should be expected that for sufficiently low temperatures, as the filling factor $\theta$ increases, the gas in the channel tends to form clusters whose size increases with the filling factor. The number and size of the clusters grow with the total number of particles in the system in a manner so as to ensure the existence

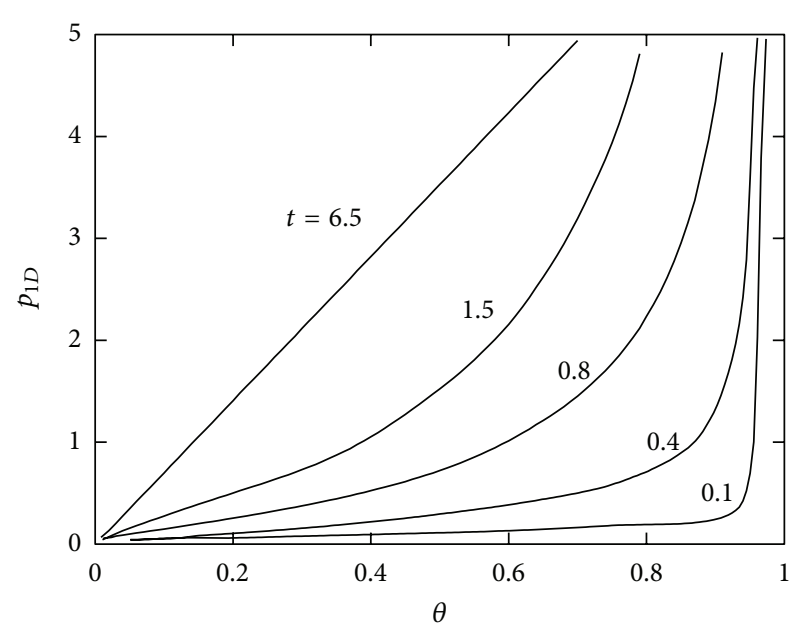

FIGURE 1: Reduced "pressure" $\left(p_{1 \mathrm{D}}\right)(24)$ of a one-dimensional gas in a channel versus the filling factor $(\theta)$ of the channel at various temperatures $t=T / T_{0}$.

of such clusters in the thermodynamic limit, where $N \rightarrow$ $\infty, L \rightarrow \infty$, and $N / L=$ const. This agrees completely with the formation of nuclei of a new phase in threedimensional systems with first-order phase transitions [34, 46]. On the other hand, in our one-dimensional system, in contrast to three-dimensional systems, a continuous behavior of thermodynamic quantities determined as derivatives of the free energy of the system with respect to the temperature, pressure, and filling factor should be expected in the entire range of variation of the thermodynamic parameters. For example, the specific heat calculated using the relation (24) will possess a maximum at the "critical" point. This fact has been observed experimentally [35], which attests to the applicability of the one-dimensional model employed previously.

2.1.2. Transport in a Dense $1 D$ System. The gas flux through a membrane is the main experimentally measurable parameter (Section 2.1.3). Particle transport is usually described using a relation between the outgoing particle flux and the parameters of the problem, such as, for example, the difference of the gas pressures on different sides of the membrane. This description is based on Fick's relation between the gas flux density $\mathbf{J}$, the gradient of the gas concentration, and the diffusion coefficient $\widetilde{D}$, using the conservation law for the number of particles:

$$
\begin{gathered}
\mathbf{J}=-\widetilde{D} \nabla n, \\
\frac{\partial n}{\partial t}+\operatorname{div} \mathbf{J}=0 .
\end{gathered}
$$

In the relations (25) and (26), $\mathbf{J}$ is the flux, $\widetilde{D}$ is the diffusion coefficient, and $\nabla n$ is the gradient of the particle concentration. The relation (25) is actually a definition of the diffusion coefficient $\widetilde{D}$. Thus, the problem of determining the particle flux through a channel reduces to calculating the diffusion coefficient. A more general method of describing 
transport is to calculate the characteristic relaxation time of density fluctuations arising or specially created in the system under study [47]. Formally, the problem of determining this time reduces to calculating the characteristic frequency or spectrum $\omega(\mathbf{k})$ for the system under study. Thus, when the particle density is low and there is no interparticle interaction, an explicit expression for the spectrum $\omega(\mathbf{k})$ can be easily obtained from the relation (25). Indeed, let $n_{0}$ be the average particle concentration in the system. From (25), we obtain an equation determining the dynamics of the density fluctuations $\delta n(\mathbf{r}, t)=n-n_{0}$ :

$$
\frac{\partial(\delta n)}{\partial t}=\widetilde{D} \Delta(\delta n)
$$

Switching to a Fourier representation of the density fluctuations

$$
\delta n(\mathbf{r}, t)=\frac{1}{(2 \pi)^{4}} \int d \mathbf{k} d \omega \exp (-i \mathbf{k} \cdot \mathbf{r}-i \omega t) \delta n(\mathbf{k}, \omega),
$$

we obtain an expression determining the reciprocal of the relaxation time of the density fluctuations $\delta n$ in the case at hand:

$$
\omega_{0}(\mathbf{k})=-i \widetilde{D} k^{2}
$$

It is obvious that a spectrum of this type is characteristic for systems where relaxation to equilibrium occurs by diffusion. Let us consider a system of interacting particles with arbitrary density. Assume that we have been able to calculate the characteristic relaxation time $\omega^{-1}$ of density fluctuations in the system. If the spectrum of relaxation times in such a system has the form

$$
\omega(\mathbf{k})=-i D\left(\mathbf{k}, n_{0}\right) k^{2},
$$

then it is natural to consider the quantity $D\left(\mathbf{k}, n_{0}\right)$ to be the diffusion coefficient in the system, provided that in the limit of low densities $n_{0}$ the quantity $D\left(\mathbf{k}, n_{0}\right)$ becomes the diffusion coefficient for a low-density system of particles:

$$
\lim _{n_{0} \rightarrow 0} D\left(\mathbf{k}, n_{0}\right)=\widetilde{D}
$$

This definition of the diffusion coefficient means that the system possesses a diffusion characteristic mode. Proceeding from the definition (30), to calculate the diffusion coefficient $D\left(\mathbf{k}, n_{0}\right)$, it is necessary to know the characteristic modes of a weakly nonequilibrium system. If these characteristic modes have the form (30), then $D\left(\mathbf{k}, n_{0}\right)$ will be the diffusion coefficient of the system. It is obvious that the diffusion coefficient in the general case is nonlocal and is a functional of the density of the diffusing particles. The expression for the flux of diffusing particles will have a form generalizing equation (25):

$$
\mathbf{J}=-\int d \mathbf{r}^{\prime} D\left(\mathbf{r}, \mathbf{r}^{\prime}, n\left(\mathbf{r}^{\prime}, t\right)\right) \nabla n\left(\mathbf{r}^{\prime}, t\right) .
$$

The equation, obtained using this relation, for the particle number density in the system is in general a nonlinear integrodifferential equation. When studying the diffusion of a single-component gas in subnanometer channels, it should be kept in mind that the pair interaction potential between the particles is of short range, and no phase transitions occur in the system of particles in the channel (see the preceding section). In this case, it can be assumed that the diffusion coefficient $D\left(\mathbf{r}, \mathbf{r}^{\prime}, n(\mathbf{r}, t)\right)$ is local:

$$
D\left(\mathbf{r}, \mathbf{r}^{\prime}, n(\mathbf{r}, t)\right)=D(n(\mathbf{r}, t)) \delta\left(\mathbf{r}-\mathbf{r}^{\prime}\right) .
$$

The corresponding expression for the flux of diffusing particles and the equation for their density become

$$
\begin{aligned}
& \mathbf{J}=-D(n) \nabla n, \\
& \frac{\partial n}{\partial t}=\nabla(D(n) \nabla n) .
\end{aligned}
$$

For low densities, the relation (34) becomes (25), which is the first term in the expansion of the flux in odd powers of the density gradient. Thus, for systems where the relaxation spectrum of the fluctuations is of the form (30), the diffusion coefficient can be determined starting from the explicit form of this spectrum. The expression obtained for the diffusion coefficient in this case is a generalization of the expressions obtained for the diffusion coefficient in various models, specifically, in the Maxwell-Stefan model [43].

It is convenient to calculate the relaxation spectrum using the response functions [31, 48, 49]. Let us formulate the basis of this formalism. Consider the system of the atomic particles, which can interact with each other as well as the environment such as liquid, solid, or surface. Let us show, following [49], that many-particles distribution functions of such a system are the density functionals. Assume that each particle at the coordinate $\mathbf{r}$ interacts with time-dependent external field $v(\mathbf{r}, t)$. In order to describe such a system, one can use Liouville equation for many-particle distribution function $F\left(x_{1}, x_{2}, \ldots, x_{N}, t\right)$, where $x_{i}=\left(\mathbf{r}_{i}, \mathbf{p}_{i}\right)$ are the coordinates and th momentum of the particles. Solving the Liouville equation with the fixed initial distribution function and for the different external potentials $V\left(\mathbf{r}_{1}, \mathbf{r}_{2}, \ldots, t\right)=$ $\sum_{i} v\left(\mathbf{r}_{i}, t\right)$, one can obtain one-to-one conformance between $F\left(x_{1}, x_{2}, \ldots, x_{N}, t\right)$ and $V\left(\mathbf{r}_{1}, \mathbf{r}_{2}, \ldots, t\right)$. On the other hand, introducing the density of the particles

$$
n(\mathbf{r}, t)=\int d \Gamma \widehat{n} F\left(x_{1}, x_{2}, \ldots, x_{N}, t\right),
$$

where $d \Gamma$ is the phase space element, and $\widehat{n}$ is the density operator, one can obtain one-to-one conformance between the external field $V\left(\mathbf{r}_{1}, \mathbf{r}_{2}, \ldots, t\right)$ and the density $n(\mathbf{r}, t)$. From this it directly follows that many-particles distribution functions are density functionals:

$$
F\left(x_{1}, x_{2}, \ldots, x_{N}, t\right)=F\left(x_{1}, x_{2}, \ldots, x_{N}, t,[n]\right) .
$$

Following [49], let us write the action for the Liouville equation in the form

$$
S=\frac{1}{t_{2}-t_{1}} \int_{t_{1}}^{t_{2}} d t \int d \Gamma L F
$$


where $L$ is the Lagrangian of the system, which is equal to the difference between the kinetic and potential energy of the particles. It follows from (36) and (37) that the action $S$ is the density functional:

$$
S=\Delta[n]=\frac{1}{t_{2}-t_{1}} \int_{t_{1}}^{t_{2}} d t \int d \Gamma L F\left(x_{1}, x_{2}, \ldots, x_{N}, t,[n]\right) .
$$

Functional $\Delta[n]$ has an extrema for the equilibrium state of the system $[48,49]$. Due to the linearity of the (38) by the potential energy, system of the particles interacting with the external field $v(\mathbf{r}, t)$ corresponds to the functional $\Delta[n, v]$, which can be expressed as

$$
\Delta[n, v]=\Delta[n]+\frac{1}{t_{2}-t_{1}} \int_{t_{1}}^{t_{2}} v(\mathbf{r}, t) n(\mathbf{r}, t) d \mathbf{r} d t .
$$

Functional (39) has an extrema for the stationary or quasistationary $n(\mathbf{r}, t)=n_{0}(\mathbf{r}, t) \equiv n^{(0)}$ state of the system $[48,49]$

$$
\left.\frac{\delta \Delta[n]}{\delta n}\right|_{n=n^{(0)}}=0
$$

and represents the time average of the free energy functional $F[n(\mathbf{r}, t), t]$ :

$$
\Delta[n]=\tau^{-1} \int_{0}^{\tau} F[n(\mathbf{r}, t), t] d t
$$

The functional $\Delta[n]$ was for the first time introduced in such a form in the papers [31, 48]. Equation (40) determines the density of the atomic particles $n(\mathbf{r}, t)$. Let us express the free energy using the response functions defined as follows. Consider the system in the external field $e v_{\text {ext }}(\mathbf{r}, t)(e-$ external field amplitude). The response function $\beta\left(\mathbf{r}, \mathbf{r}^{\prime}, t, t^{\prime}\right)$ of such a system couples the density fluctuation $\delta n(\mathbf{r}, t)$ with the external field $e v_{\text {ext }}(\mathbf{r}, t)$, which generate this fluctuation $[31,48]$ at $e \rightarrow 0$ :

$$
\delta n(\mathbf{r}, t)=\left.\int d \mathbf{r}^{\prime} d t^{\prime} \beta\left(\mathbf{r}, \mathbf{r}^{\prime}, t, t^{\prime}\right) e v_{\mathrm{ext}}\left(\mathbf{r}^{\prime}, t^{\prime}\right)\right|_{e \rightarrow 0}
$$

It is obvious that in the case of the linear response and $e \rightarrow 0$, the response function $\beta\left(\mathbf{r}, \mathbf{r}^{\prime}, t, t^{\prime}\right)$ is independent on $e$. Let us rewrite the definition (42) in the operator form

$$
\delta n=e v_{\text {ext }} \beta .
$$

Such a form (43) will be used further in the paper. In the presence of the external field, the functional $\Delta[n, v]$ at $e \rightarrow 0$ is determined by the relation (39):

$$
\Delta\left[n, e v_{\mathrm{ext}}\right]=\Delta[n]+\frac{1}{t_{2}-t_{1}} \int_{t_{1}}^{t_{2}} e v_{\mathrm{ext}}(\mathbf{r}, t) n(\mathbf{r}, t) d \mathbf{r} d t
$$

Here $\Delta[n]$ is the functional at $e=0$, determined by the formula (38). In order to obtain linear response function, let us use the relation (39). One can rewrite the solution of the (39) at $e \rightarrow 0$ with the sum $n(\mathbf{r}, t)=n^{(0)}(\mathbf{r}, t)+\delta n(\mathbf{r}, t)$. In this case, one can obtain, in the first order and with respect to (39),

$$
\left.\frac{\delta \Delta[n]}{\delta n}\right|_{n=n^{(0)}}+\left.\frac{\delta^{2} \Delta[n]}{\delta n^{2}}\right|_{n=n^{(0)}} \delta n+e v_{\mathrm{ext}}=0
$$

or

$$
-\left(\left.\frac{\delta^{2} \Delta[n]}{\delta n^{2}}\right|_{n=n^{(0)}}\right)^{-1} e v_{\mathrm{ext}}=\delta n .
$$

At $e \rightarrow 0$, the quantity

$$
\left.\frac{\delta^{2} \Delta[n]}{\delta n^{2}}\right|_{n=n^{(0)}} \equiv \frac{\delta^{2} \Delta}{\delta n(\mathbf{r}, t) \delta n\left(\mathbf{r}^{\prime}, t^{\prime}\right)}
$$

is independent on e. Comparison of the relations (43) and (46) gives the following form of the response function $\beta\left(\mathbf{r}, \mathbf{r}^{\prime}, t, t^{\prime}\right)$ :

$$
\begin{aligned}
\beta\left(\mathbf{r}, \mathbf{r}^{\prime}, t, t^{\prime}\right) & =-\left(\left.\frac{\delta^{2} \Delta[n]}{\delta n^{2}}\right|_{n=n^{(0)}}\right)^{-1} \\
& =-\left(\frac{\delta^{2} \Delta}{\delta n(\mathbf{r}, t) \delta n\left(\mathbf{r}^{\prime}, t^{\prime}\right)}\right)^{-1} .
\end{aligned}
$$

It follows from the functional $\Delta[n]$ definition that it can be expressed as

$$
\Delta[n]=\Delta_{0}[n]+\Delta_{\text {int }}[n] .
$$

Here $\Delta_{0}[n]$ corresponds to the functional of the particles without their interaction with each other, and $\Delta_{\text {int }}[n]$ corresponds to the functional (38), which represents particleparticle interactions. Two times variation of the (49), with respect to (48), gives the following:

$$
-\beta^{-1}=-\beta_{0}^{-1}+R,
$$

where

$$
\beta_{0}^{-1}\left(\mathbf{r}, \mathbf{r}^{\prime}, t, t^{\prime}\right)=-\left.\frac{\delta^{2} \Delta_{0}[n]}{\delta n^{2}}\right|_{n=n^{(0)}}=-\frac{\delta^{2} \Delta_{0}}{\delta n(\mathbf{r}, t) \delta n\left(\mathbf{r}^{\prime}, t^{\prime}\right)}
$$

is the response function of the noninteracting particles

$$
R\left(\mathbf{r}, \mathbf{r}^{\prime}, t, t^{\prime}\right)=-\left.\frac{\delta^{2} \Delta_{\mathrm{int}}[n]}{\delta n^{2}}\right|_{n=n^{(0)}}=-\frac{\delta^{2} \Delta_{\mathrm{int}}}{\delta n(\mathbf{r}, t) \delta n\left(\mathbf{r}^{\prime}, t^{\prime}\right)} .
$$

It is shown in the paper $[31,48]$ that the function $R\left(\mathbf{r}, \mathbf{r}^{\prime}, t, t^{\prime}\right)$ is connected to the effective lagged interaction between the particles. Multiplying the relation (45) on the $\beta$ in the left and on the $\beta_{0}$ in the right, one can obtain the equation on the response function. In the case of the homogeneous quasi-stationary state of the atomic system, the functions $\beta_{0}\left(\mathbf{r}, \mathbf{r}^{\prime}, t, t^{\prime}\right), \beta\left(\mathbf{r}, \mathbf{r}^{\prime}, t, t^{\prime}\right)$ и $R\left(\mathbf{r}, \mathbf{r}^{\prime}, t, t^{\prime}\right)$ depend only on the differences $\mathbf{r}-\mathbf{r}^{\prime}$ и $t-t^{\prime}$, so (50) can be rewritten as

$$
\beta(\mathbf{k}, \omega)=\beta_{0}(\mathbf{k}, \omega)+\beta_{0}(\mathbf{k}, \omega) R(\mathbf{k}, \omega) \beta(\mathbf{k}, \omega),
$$


where $\beta(\mathbf{k}, \omega), \beta_{0}(\mathbf{k}, \omega)$ и $R(\mathbf{k}, \omega)$ are the Fourier transform of the response functions $\beta\left(\mathbf{r}-\mathbf{r}^{\prime}, t-t^{\prime}\right), \beta_{0}\left(\mathbf{r}-\mathbf{r}^{\prime}, t-t^{\prime}\right)$, and $R\left(\mathbf{r}-\mathbf{r}^{\prime}, t-t^{\prime}\right)$, respectively; for example,

$$
\beta_{0}\left(\mathbf{r}-\mathbf{r}^{\prime}, t-t^{\prime}\right)=\int d \mathbf{k} d \omega e^{-i \omega\left(t-t^{\prime}\right)+i \mathbf{k}\left(\mathbf{r}-\mathbf{r}^{\prime}\right)} \beta_{0}(\mathbf{k}, \omega) .
$$

Equation (53) allows us (when the $R$ function is known) to determine the response function of the system of interacting particles and relaxation spectrum of the system [31, 48].

In order to determine the relaxation spectrum, let us note, that the expression $\beta^{-1} \delta n=e v_{\text {ext }}$ follows directly from (43). Due to the response function definition (as the response of the system on the arbitrary small external field), one can write $\beta^{-1} \delta n=0$. This equation has the nontrivial solutions $\delta n \neq 0$ only if $\beta^{-1}=0$. So, we can write that the relaxation spectrum of the system $\omega(\mathbf{k})$ is determined by the relation $[31,48]$

$$
\beta^{-1}(\mathbf{k}, \omega)=0 .
$$

We will use the response function formalism to calculate the response function and the diffusion coefficient of a dense system of atomic particles in a subnanometer channel. We will calculate first the response function $\beta_{0}(k, \omega)$ and the relaxation spectrum of such a gas when there is no interaction between the particles. We write the diffusion equation for the filling factor $\theta$ of the channel with molecules in the presence of a weak perturbing external field $e v_{\text {ext }}(x, t)$ in the form

$$
\frac{\partial \theta}{\partial t}=D_{0} \nabla\left(\nabla \theta+\frac{\theta}{T} \nabla e v_{\text {ext }}\right), \quad \nabla \equiv \frac{\partial}{\partial x} .
$$

Here $D_{0}$ is the diffusion coefficient for noninteracting particles. Let $\theta_{0}$ be the equilibrium filling factor of the channel. We will seek the solution of (56) in the form

$$
\theta=\theta_{0}+\delta \theta(x, t)
$$

Then, we obtain from (56), assuming the external field $e_{\text {ext }}$ to be weak, an equation for $\delta \theta(r, t)$ :

$$
\frac{\partial \delta \theta}{\partial t}=D_{0} \Delta \delta \theta+\frac{D_{0} \theta_{0} \Delta e v_{\mathrm{ext}}}{T} .
$$

In deriving (58), only first-order infinitesimals in $\delta \theta$ and $e v_{\text {ext }}$ were retained in all terms. We will seek the solution of (58) in the form of a Fourier integral

$$
\begin{gathered}
\delta \theta(x, t)=\frac{1}{(2 \pi)^{2}} \int e^{i k \cdot x} e^{i \omega t} \delta \theta(k, \omega) d k d \omega, \\
e v_{\mathrm{ext}}(x, t)=\frac{1}{(2 \pi)^{2}} \int e^{i k \cdot x} e^{i \omega t} e v_{\mathrm{ext}}(k, \omega) d k d \omega .
\end{gathered}
$$

Substituting the expressions (59) into (58) and solving the resulting linear equation for $\delta \theta(k, \omega)$, we obtain

$$
\delta \theta(k, \omega)=\beta_{0}(k, \omega) e v_{\mathrm{ext}}(k, \omega) .
$$

Here

$$
\beta_{0}(k, \omega)=-\frac{\theta_{0} k^{2} D}{T\left(i \omega+k^{2} D_{0}\right)}
$$

is the response function of a gas of noninteracting diffusing particles. Indeed, as $\omega \rightarrow 0$, the function $\beta_{0}(k, \omega)$ reduces to the well-known response function of an ideal equilibrium gas with density $\theta_{0}$ at temperature $T[38,47]$ :

$$
\beta_{0}(k, 0)=-\frac{\theta_{0}}{T} \text {. }
$$

The relaxation spectrum of such a gas is determined from the relation (55) and has the form

$$
\omega_{0}(k)=-i D_{0} k^{2} .
$$

In accordance with the definition (26), the quantity $D_{0}$ is the diffusion coefficient. The relations obtained make it possible to calculate the relaxation spectrum $\omega(k)$ by solving (54). Thus, we find for the response function of a one-dimensional system

$$
\beta(k, \omega)=\frac{\beta_{0}(k, \omega)}{1-\beta_{0}(k, \omega) R(k, \omega)} .
$$

Using the relations (61) and (64), we obtain an equation determining the relaxation spectrum of the system under study:

$$
1+\frac{\theta_{0}}{T} \frac{\omega_{0}}{\omega+\omega_{0}} R(k, \omega)=0 .
$$

Equation (65) can be solved in a general form in the quasistatic case $\omega \rightarrow 0$. Indeed, in this case, we find from (65)

$$
\begin{gathered}
\omega(k)=-i D\left(\theta_{0}, k\right) k^{2}, \\
D\left(\theta_{0}, k\right)=D_{0}\left[1+\frac{\theta_{0}}{T} R(k, 0)\right] .
\end{gathered}
$$

It is convenient to rewrite the relation (67), introducing the pair distribution function $n_{2}\left(x-x^{\prime}\right)=\theta_{0}^{2}\left[1+\nu\left(x-x^{\prime}\right)\right]$ :

$$
T \beta(k, 0)=-\theta_{0}\left(1+\theta_{0} \nu(k)\right) \text {. }
$$

Then we obtain from relations (53) and (61)

$$
R(k, 0)=-\frac{T \nu(k)}{1+\theta_{0} \nu(k)} .
$$

Substituting the expression (69) into (67) gives a relation determining the relaxation spectrum of a dense gas in a onedimensional channel:

$$
\omega(k)=-\frac{i D_{0} k^{2}}{1+\theta_{0} \nu(k)} .
$$

We will now calculate the diffusion coefficient of a gas in a one-dimensional channel taking into account the interaction between hard-sphere particles. In this case, the correlation function $n_{2}(x)=\theta_{0}^{2}[1+\nu(x)]$ has been calculated exactly [45] with an arbitrary filling factor of the channel:

$$
\begin{aligned}
& n_{2}(x) \\
&= \begin{cases}0, & r<\sigma, \\
\frac{1}{\theta_{0}} \sum_{m} \frac{\left(x \sigma^{-1}-m\right)^{m-1}}{(m-1) !\left(\theta_{0}^{-1}-1\right)^{m}} \exp \left(-\frac{x \sigma^{-1}-m}{\theta_{0}^{-1}-1}\right), & x>\sigma .\end{cases}
\end{aligned}
$$


Using the relations (70) and (71), we find

$$
\begin{aligned}
& \omega(k)=-i D\left(\theta_{0}\right) k^{2}, \\
& D\left(\theta_{0}\right)=D_{0}\left(\frac{1}{\left(1-\theta_{0}\right)^{2}}\right) .
\end{aligned}
$$

It follows from (72) that the general equation describing particle transport in a dense $1 \mathrm{D}$ system, in accordance with (34), has the form

$$
\frac{\partial \theta}{\partial t}=\frac{\partial}{\partial x}\left[D_{0}\left(\frac{1}{(1-\theta)^{2}}\right) \frac{\partial \theta}{\partial x}\right] .
$$

This equation can be obtained from (72) if the characteristic local equilibration times in a dense system of particles in a channel are short compared with the characteristic propagation times of disturbances along such a system. For the cases considered in the following, this assumption obviously holds. We will consider solutions of (73) in the case where complete equilibrium has been established in the channel and the filling factor $\theta=\theta_{0}(p, T)$, with the function $\theta_{0}(p, T)$ being determined by the relation (23). Transport in a channel in this case will be determined by the dynamics of the motion of disturbances of the filling factor. The solution of (73) in this case should be sought in the form $\theta=\theta_{0}(p, T)+\delta \theta(x, t)$. It follows from (73) that the relaxation spectrum of disturbances in this case has the form (72), where $\theta=\theta_{0}(p, T)$. Thus, taking account of the interaction of hard-sphere particles in a channel does not change the character of the relaxation of its weakly nonequilibrium state: the relaxation spectrum (72) remains a diffusion spectrum with diffusion coefficient $D\left(\theta_{0}\right)$. However, it is important to underscore that transport in the channel in this case is a collective effect and proceeds via transport of disturbances of the equilibrium density $\theta_{0}(p, T)$. The diffusion coefficient $D\left(\theta_{0}\right)$ in this case is the diffusion coefficient of disturbances of the equilibrium density. It is convenient to rewrite the relation (72) for the diffusion coefficient in a different form. Using the Arrhenius character of the diffusion coefficient for noninteracting particles

$$
D_{0}=\widetilde{D}_{0} \exp \left(-\frac{E}{T}\right)
$$

( $\widetilde{D_{0}}$ is proportional to the product of the squared lattice constant of the wall material of the channel and the relaxation frequency of particles in a channel on defects and lattice phonons and is calculated in [37], and $E \approx \Psi\left(r_{\max }\right)$ (13) is the activation energy of diffusing of noninteracting particles in the channel), we will rewrite (72) in the form

$$
\begin{aligned}
& D\left(\theta_{0}\right)=\widetilde{D}_{0} \exp \left(-\frac{\widetilde{E}\left(\theta_{0}\right)}{T}\right), \\
& \widetilde{E}\left(\theta_{0}\right) \equiv E-T \ln \left(\frac{1}{\left(1-\theta_{0}\right)^{2}}\right) .
\end{aligned}
$$

This way of writing the diffusion coefficient for a gas of interacting particles in a channel makes it possible to give a physical interpretation for the change in the diffusion coefficient accompanying a change in the filling factor $\theta_{0}$. The relation (75) shows that the interaction of particles in a channel decreases the activation energy $E$ of the motion of particles, even in the hard-sphere model. For an interaction between hard-sphere particles such that there is no direct attraction between the particles, the diffusion activation energy decreases with increasing filling factor $\theta_{0}$ as a result of the effective interaction (see the following). Physically, this corresponds to a change because of the presence of another particle in a neighboring potential well, in the parameters of the potential in which a gas particle moves. Since the gas particles are assumed to be indistinguishable, diffusion with the filling factor $\theta_{0} \sim 1$ (when the effective diffusion activation energy $\widetilde{E}\left(\theta_{0}\right)$ becomes comparable to the temperature of the system) can be interpreted as a transfer of "excitation" of the density along a chain of close gas particles. It is obvious that the motion of such an "excitation" will occur with substantial velocities. This leads to a large increase in the diffusion coefficient when $\theta_{0} \sim 1$. For $E=0$, the possibility of such a diffusion mechanism is played out in [36]. We note that for all gases investigated, the barrier $E$ is different from 0 . This is indicated by the presence of a maximum in the temperature dependence of the flux in a zeolite membrane [43]. It can be assumed that the increase in the diffusion coefficient in a channel observed with filling factors $\theta_{0} \geq 0.5$ is related to the formation of clusters in the channel whose sizes increase with the filling factor. The transport of gas in the channel containing such clusters is determined by the motion of "excitation" in a finite-size cluster. The formation of clusters in a gas consisting of particles with a hard-sphere pair interaction potential is related to the well-known [46] appearance of an effective attraction between such particles. This effect is manifested in the appearance of a maximum in the correlation function $n_{2}(x)$, describing the probability of finding the "first" particle at a distance $x$ from the "second" particle.

The relaxation spectra of the system for an arbitrary wave vector $k$ can be found from (70) and (71). It is found that the spectrum $\omega(k)$ contains a real part, which corresponds to transport of an "excitation" in the system. The imaginary part of the spectrum obtained has a minimum at a definite value of the wave vector $k=k_{\min }$, which depends on the filling factor $\theta_{0}$. It is natural to interpret the value $R=$ $2 \pi / k_{\min }\left(\theta_{0}\right)$ as the characteristic size of a cluster with a given filling factor and the value $\tau=\operatorname{Im} \omega^{-1}\left(k_{\min }\left(\theta_{0}\right)\right)$ as its characteristic lifetime. As the filling factor increases, the value of the effective attraction between the particles increases [46]; this could lead to the appearance of clusters consisting of two or more particles. However, it is found that as a result of the one-dimensionality of the channel, the lifetime of the clusters that are formed is finite, which corresponds to the absence, as noted previously, of a phase transition in such systems. The dependences of the lifetime and size of the clusters formed on the filling factor $\theta_{0}$ are presented in Figure 2 .

It is evident in the figure that increasing the filling factor increases the lifetime and the size of the clusters formed. In finite-size channels, for a definite value of the filling factor 


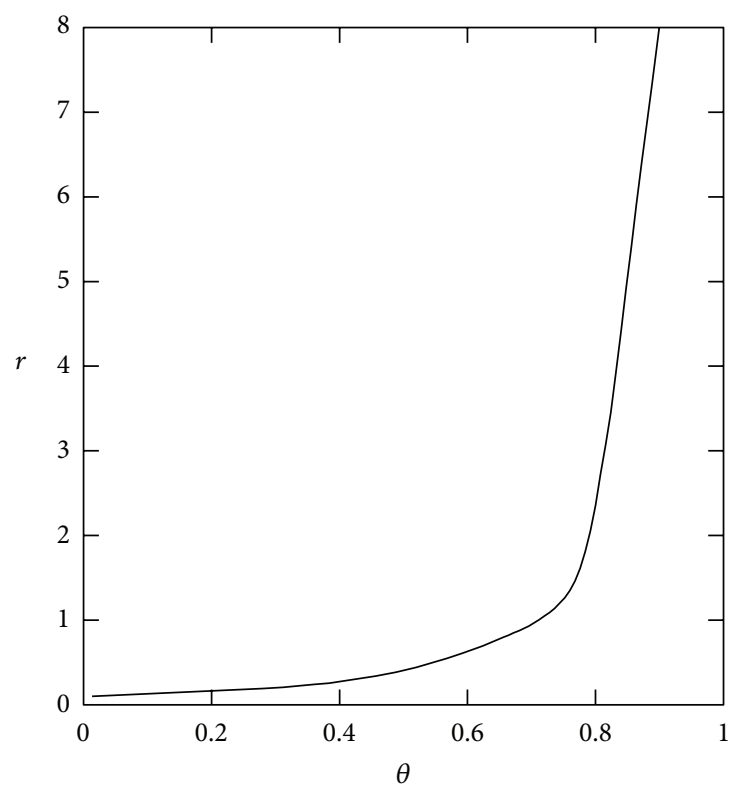

(a)

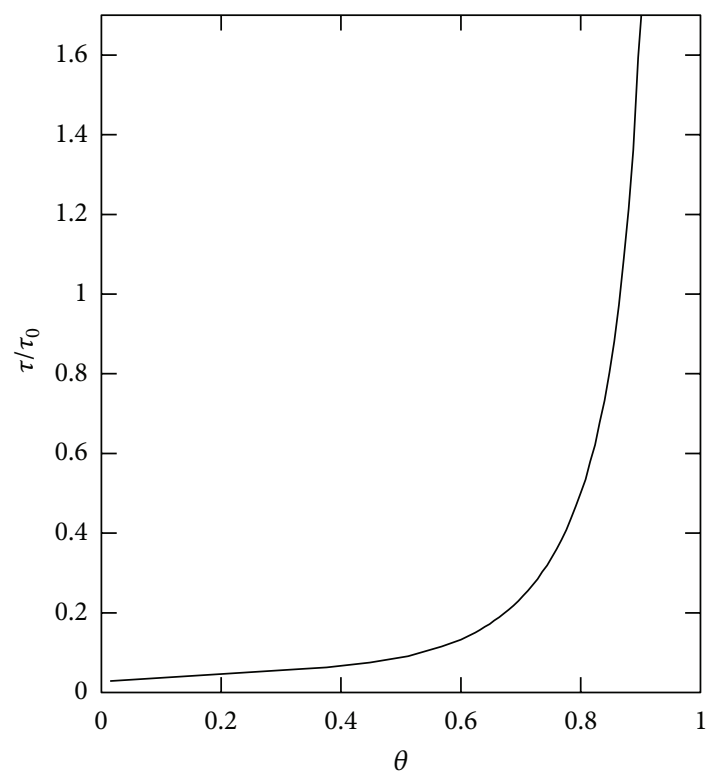

(b)

Figure 2: Size $(r=R / \sigma)$ (a) and lifetime $\left(\tau / \tau_{0}, \tau_{0}=\sigma^{2} / D_{0}\right)$ (b) of clusters versus the filling factor.

$\theta_{0}=\theta_{c}$, a cluster equal in size to the length of the channel is formed. It is obvious that the diffusion coefficient in such a channel increases without bound as $\theta_{0} \rightarrow \theta_{c}$, even though the lifetime of such a cluster is finite. If the range of relaxation frequencies $\omega(k)$ is known, then an expression that defines the relaxation of the Fourier components of the particle density in the channel $n(k, t)$ in the case of arbitrary density $n(k, t)$ is given by

$$
\dot{n}(k, t)=i \omega(k) n(k, t) \text {. }
$$

From (76), we can get that the equation for the amplitude of the fluctuations can be written as

$$
\delta \dot{n}(k, t)=i \omega(k) \delta n(k, t) .
$$

Equation (77) describes the relaxation of $k$ component of the density fluctuation at arbitrary wave vector. In particular, at $k \neq 0$, this equation allows to describe the relaxation of density fluctuations and propagation of disturbances on the cluster of finite size in the case of the formation of the cluster. At $k \rightarrow 0$, the equation describes the relaxation of density fluctuations on large spatial scales. This value is related to the macroscopic flux. Let us write the equation of continuity to calculate the flux $j$ :

$$
\dot{n}+\frac{\partial j}{\partial x}=0 .
$$

After applying the Fourier transform, (78) takes the form

$$
i k j(k, t)=-\dot{n}(k, t) .
$$

Using (76) and (79), one can get for $j(k)$

$$
j(k, t)=\frac{n(k, t) \omega(k)}{k} .
$$

Using the relation (70) we find that $v(k)$ and therefore the spectrum contains both real and imaginary parts, corresponding to the "hydrodynamic" (real part of $\omega(k)$ ) and diffusive (imaginary part of $\omega(k)$ ) transport of particles. Therefore, $(80)$ for the flux $j(k, t)$ contains terms relating to hydrodynamic transport of particles in a cluster and diffusive transport of particles in the space between clusters. We note that the response function method can be used to take into account the interaction of particles in a channel for a potential that is different from a hard-spheres interaction, similarly to the way this was done previously.

Analysis of the experimental data showed that for high filling factors $\theta_{0} \sim 1$, the dependence of the diffusion coefficient $D\left(\theta_{0}\right)$, calculated from the relation (72), leads to a discrepancy between theory and experiment (Section 2.1.3). This discrepancy could be due, in our opinion, to the abovedescribed influence of a finite channel length, the asphericity of the gas molecules, and the possible breakdown of one dimensionality of the problem. These effects lead to the following dependence of the diffusion coefficient on the filling factor:

$$
D\left(\theta_{0}\right)=D_{0}\left(\frac{1}{\left(1-\zeta \theta_{0}\right)^{2}}\right) .
$$

Here $\zeta$ is a coefficient that takes into account the finite size of the channel, the possible deviation of the channel from one-dimensionality, and the asphericity of the molecules. The relations (72), (81), and (52) make it possible to describe transport in a subnanometer channel for different values of parameters such as the gas pressure and the pressure difference outside the membrane, the temperature of the membrane, and the type of gas molecules. As an example, we present the dependence, calculated according 


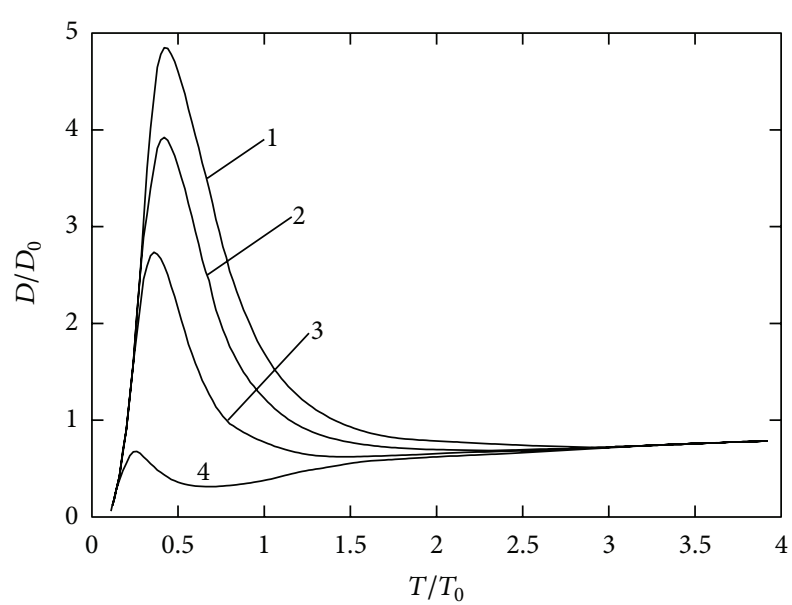

FIGURE 3: Theoretical curves of the relative diffusion coefficient $D / D_{0}$ versus the relative temperature $T / T_{0}$ for various values of the relative pressure $p / p_{0}$ : (1) $p / p_{0}=3$, (2) $p / p_{0}=2$, (3) $p / p_{0}=1$, and (4) $p / p_{0}=0.1$.

to (72), (81), and (23), of the diffusion coefficient in a subnanometer channel on the relative temperature $T / T_{0}$ for various relative pressures $p / p_{0}$ (Figure 3 ).

It is evident in the figure that the character of the temperature dependence of the relative diffusion coefficient in subnanometer channels is substantially different at different pressures $p$. For example, if the pressure is high $\left(p / p_{0}>\right.$ 1 ), the dependence of $D / D_{0}$ on $T / T_{0}$ has a pronounced maximum at temperatures $T \sim 0.5 T_{0}$ (curve 1 ). As pressure decreases, the magnitude of this maximum decreases and the maximum itself shifts into the region of lower temperatures (curves 2,3). A further decrease of pressure to values $p / p_{0}<$ 1 results in the appearance of a minimum in the dependence of $D / D_{0}$ on $T / T_{0}$ in the temperature range $T \sim 0.5 T_{0}$. The maximum occurs at $T \sim 0.3 T_{0}$. Since for $p / p_{0}<1$, the flux depends on $p$ and the average distance between particles in the channel is much greater than the diameter of the particles, the increase in the diffusion coefficient for $T \sim$ $0.5 T_{0}$ is due to the temperature dependence of the diffusion coefficient $D_{0}$ on individual molecules in the channel. At high temperatures, all curves saturate at a value corresponding to $D / D_{0}=1$. In the next section, the theoretical laws obtained previously will be compared with the experimental data.

2.1.3. Analysis of the Experimental Data: Comparison of Theory with Experiment. Quite extensive experimental investigations of the sorption and transport properties of a series of organic molecules $\left(\mathrm{CH}_{4}, \mathrm{C}_{2} \mathrm{H}_{6}, n-\mathrm{C}_{4} \mathrm{H}_{8}, i-\mathrm{C}_{4} \mathrm{H}_{8}\right.$, and others) and the inert gases $\mathrm{Ar}, \mathrm{Ne}$, and $\mathrm{Kr}$ in ZSM-5 zeolite membranes (MFI, silicate-1) have now been performed. The results are presented in [42, 50, 51]. According to [52], ZSM-5 zeolite membranes have a complicated chemical and crystalline structure. The chemical structure of zeolite membranes is given by the formula

$$
\mathrm{Na}_{n}\left[\mathrm{Si}_{96-n} \mathrm{Al}_{n} \mathrm{O}_{192}\right] \cdot 16 \mathrm{H}_{2} \mathrm{O}, \quad n \leq 8 .
$$

For small values of $n$, zeolite membranes of this type are called silicate- 1 membranes. The crystalline structure of ZSM5 membranes has been studied quite well. It consists of a 3D structure, consisting of sinusoidal channels with a circular cross section $(0.54 \pm 0.02 \mathrm{~nm})$ parallel to the $a$ axis [53], which intersect straight channels with an elliptical cross section $(0.57-0.58) \times(0.51-09.52) \mathrm{nm}^{2}$ parallel to the $b$ axis [010] $[50,54]$. Cavities $\sim 0.9 \mathrm{~nm}$ in size form at the intersection of the channels. The sorption capacity of ZSM-5 zeolite is determined by the number of sorbed (entering the channels) molecules per cell of a crystal. According to $[50,55]$, the cell parameters are $a=2.007 \mathrm{~nm}, b=1.992 \mathrm{~nm}$, and $c=1.342 \mathrm{~nm}$. A single cell is a structure consisting of four segments of $0.46 \mathrm{~nm}$ linear channels, four segments of $0.66 \mathrm{~nm}$ sinusoidal channels, and four $0.54 \mathrm{~nm}$ intersections. Depending on the structure of a molecule, sorption of one or two molecules per intersection is possible. In [50], the sorption capacity of ZSM-5 was measured for a number of molecules. It was concluded that the molecules $\mathrm{CH}_{4}, \mathrm{C}_{3} \mathrm{H}_{8}$, and $n-\mathrm{C}_{4} \mathrm{H}_{10}$ are sorbed with one molecule per intersection, while nitrogen, $n$-hexane, and $p$-xylene are sorbed with two molecules per intersection. Thus, for gases of the type $\mathrm{CH}_{4}$, $n-\mathrm{C}_{3} \mathrm{H}_{8}$, and $\mathrm{C}_{4} \mathrm{H}_{10}$, channels in the crystal structure of ZSM- 8 can be treated as one dimensional. For nitrogen-type molecules, two molecules can be arranged at an intersection, and therefore molecules can change places at an intersection (absence of blocking). Nonetheless, since there are significantly more nitrogen molecules in a channel (24 molecules per unit cell [50]), the one-dimensional model can also be used for this gas. Analysis of the experimental data presented $[43,55-58]$ shows that all gases investigated can be conditionally divided into two groups. The first group of gases, containing, specifically, $\mathrm{Ar}, \mathrm{Kr}, \mathrm{Ne}$, and $\mathrm{CH}_{4}$, is characterized by a linear pressure dependence of the filling factor $\theta$; that is, Henry's law holds for them [43]. For these gases, the pressure dependence of the flux is nearly linear, and the diffusion coefficient is essentially independent of the filling factor [55]. The temperature dependence of the flux is characterized by the presence of a minimum at temperatures $T \sim 400 \mathrm{~K}$ (e.g., for $\mathrm{Ar}$ and $\mathrm{Kr}$ ), while a maximum is not observed in the diffusion coefficient $[57,58]$. Here neon, whose diffusion coefficient increases with temperature [58], is an exception. The second group of gases, containing, specifically, $\mathrm{i}-\mathrm{C}_{4} \mathrm{H}_{10}$, $\mathrm{C}_{2} \mathrm{H}_{4}, \mathrm{C}_{2} \mathrm{H}_{6}$, and $\mathrm{C}_{4} \mathrm{H}_{8}$, is characterized by a dependence of the filling factor $\theta$ on the pressure $p$ in the form of a curve that saturates [43]. For these gases, the pressure dependence of the flux is likewise characterized by a curve that emerges at a value that is pressure independent [55]. For this group of gases, the diffusion coefficient depends strongly on the filling factor [56]. The temperature dependence of the flux is characterized by the existence of a maximum and minimum at high $(T>500 \mathrm{~K})$ temperature [57]. The model, constructed in this paper, of the sorption and transport properties makes it possible to describe these dependences of transport in onedimensional channels on the basis of general assumptions. Figure 4 shows the dependence of the diffusion coefficient $D$ on the filling factor $\theta$ (solid line), calculated using (81) and the experimental data obtained in [57] for various gases. 


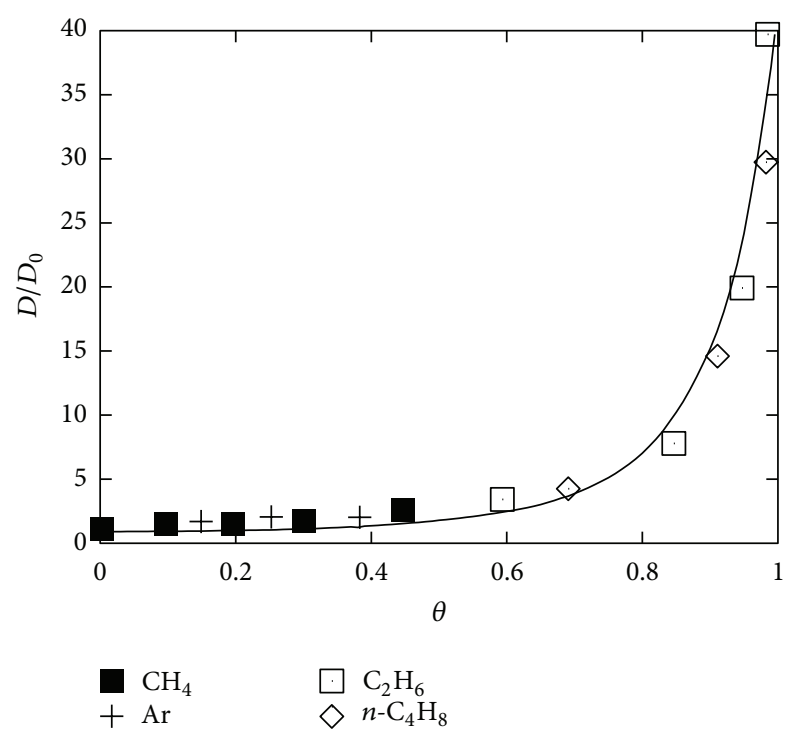

FIGURE 4: Relative diffusion coefficient versus the filling factor. Solid curve-theoretical dependence (81) with $\zeta=0.86$; dotsexperimental data from [57].

We note that the dependence (81) presented in Figure 4 takes into account the asphericity of the molecules, equal to $\zeta=$ 0.86. For inert gases and methane (first group of gases), the diffusion coefficient measured in the range of filling factors $\theta_{0}<0.5$ depends weakly on $\theta_{0}$. A large increase in the diffusion coefficient at $\theta_{0} \sim 1$ is observed for gases of the type $\mathrm{C}_{2} \mathrm{H}_{6}$ and $n-\mathrm{C}_{4} \mathrm{H}_{8}$ (second group of gases). This behavior of the diffusion coefficient $D(\theta)$ can be satisfactorily described on the basis of the model proposed, which takes account of the pair interaction of particles with a very simple form of the interaction potential for hard spheres. It is obvious that this coefficient is different for different molecules.

The physical mechanism leading to an increase of the diffusion coefficient consists in a decrease of the activation barriers for diffusion, which is due to an interaction of the particles for high filling factors. Indeed, it follows from the relation (75) for the effective diffusion activation energy $\widetilde{E}\left(\theta_{0}\right)$ that in this case, when the filling factor is low, $\theta_{0} \ll 1$, gas diffusion in the channel can be studied in the singleparticle approximation neglecting the pair interaction of the gas particles with one another. As the filling factor increases, the diffusion activation energy decreases as a result of the effective attraction of the gas particles to one another. This can be interpreted as the formation of widely separated clusters. Diffusion in each cluster can be treated as a transfer of "excitation" along a chain of close gas particles. A further increase of the filling factor increases the cluster lifetime and decreases the average intercluster distance and, in consequence, increases the diffusion coefficient. For $\theta_{0} \sim$ 1 , the diffusion process can be regarded as a motion of this "excitation" along the entire channel, when the arrival of a particle at the channel entrance results in the end-most particle leaving the channel.

Figure 5(a) shows the temperature dependences of the gas flux through a membrane for ethane and argon, calculated using the formulas (5) and (81) and a relation following from (81): $J \propto D\left(\theta_{0}(T, p)\right) \theta_{0}(T, p)$. These dependences were compared with the experimental values obtained in [42]. It is evident that the theoretical and experimental results agree satisfactorily with one another. The temperature dependence can also be understood on the basis of the model developed in the present work. Indeed, it follows from the relation (5) that at low temperatures $(T \rightarrow 0)$, the filling factor $\theta_{0} \sim 1$.

The effective diffusion activation energy $\widetilde{E}\left(\theta_{0}\right)$ is $E_{0}$ at zero temperature (56). The diffusion coefficient of the gas particles in the channel (81) approaches zero as a result of the "freezing out" of the thermal motion of the particles in the potential field of the channel surface. Increasing the temperature decreases $\widetilde{E}\left(\theta_{0}\right)$ without appreciably changing the filling factor. The value of $\widetilde{E}\left(\theta_{0}\right)$ reaches a minimum at a certain temperature $T_{0}$. It is obvious that the diffusion coefficient reaches its maximum value precisely at this temperature. It follows from (54) and (75) that a further increase of temperature decreases the filling factor $\theta_{0}$, and this is accompanied by an increase in the effective diffusion activation energy right up to the value $E_{0}$ which is attained for $\theta_{0}(p, T) \approx \theta_{0} \ll 1$ at some temperature $T=T_{1}(p)$. The temperature $T_{1}$ corresponds to a minimum in the function $D(T)$. A further increase of the diffusion coefficient with increasing temperature corresponds to the variation of the coefficient according to the Arrhenius law $D \propto \exp \left(-E_{0} / T\right)$. Figure 5(b) shows the temperature dependence of the flux for argon, calculated using (81) and the data from [57]. We note that the theory developed in the present paper predicts that the presence of a minimum at $T \sim 400 \mathrm{~K}$ should be accompanied by the appearance of a maximum at $T \sim 200 \mathrm{~K}$. In this connection, for further elaboration of the theory, it is of interest to make an experimental search for a maximum in the temperature dependence of the flux for argon near $T \sim 200 \mathrm{~K}$. Thus, two types of behavior of particles in a channel, which have different diffusion mechanisms, can be distinguished in the pressure and temperature ranges investigated. For the first group, this is the diffusion of single particles in a channel, and for the second group it is diffusion as a result of a collective interaction of particles in a completely filled channel. Such behavior of single-component gases is a direct consequence of the real one-dimensionality of subnanometer channels, where molecules cannot change places with one another. The proposed model is based on two basic assumptions: (1) the pair interaction between gas particles plays the decisive role in the description of the state and transport phenomena in a one-component gas in subnanometer channels, and (2) as a result of the fact that the channel diameter is comparable to the diameter of the gas molecules, such channels can be assumed to be one dimensional. The one-dimensionality of the system studied is actually due to the fact that the gas molecules do not have classical transverse degrees of freedom. This difference from the conventional systems plays a fundamental role in the analysis of all phenomena described above.

We note that (73) derived previously possesses, besides the solution described in the text, strongly nonuniform nonstationary soliton-like solutions. It should be expected 


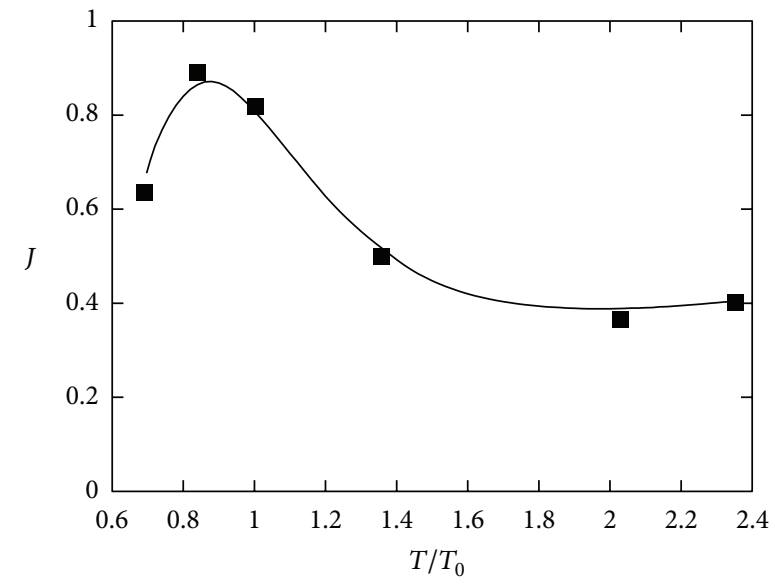

(a)

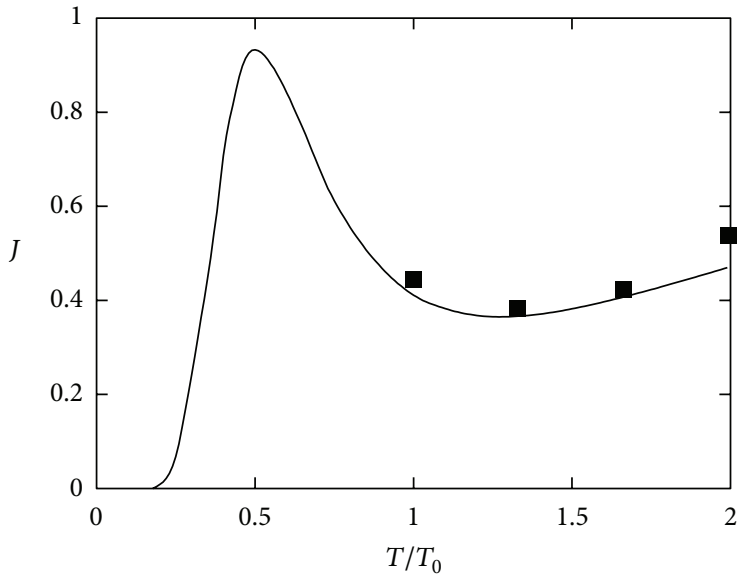

(b)

FIgURE 5: Relative flux versus the relative temperature $T / T_{0}, T_{0}=296 \mathrm{~K}$. Solid lines-theoretical curves calculated using (5), (34), and (81); dots-experimental data for ethane (a) [42] and argon (b) [58].

that an experimental consequence of the existence of such solutions will be the appearance of substantial fluctuations of the gas flux through a membrane. The proposed model satisfactorily describes the equilibrium properties of a gas in a channel (specifically, the filling factor) and the weakly nonequilibrium properties of the gas, such as the diffusion coefficient and flux. It should be noted that a satisfactory description of the equilibrium properties, for example, the adsorption isotherm, has been obtained previously on the basis of a number of phenomenological models [42]. A qualitative analysis of the maximum (but not minimum) in the temperature dependence of the relative diffusion coefficient is presented in [43]. A model describing the dependence of the diffusion coefficient on the filling factor on the basis of a oneparticle approximation by introducing into the diffusion rate jumps of individual particles a phenomenological correction factor $\chi=\left(1-\theta_{0}\right)^{-1}$ has been proposed in [36]. However, even though each of the phenomena indicated previously can be described on the basis of individual models, the approximations used in so doing either are not physically substantiated or completely contradict one another.

2.2. Particle Mobility and Diffusion in $1 D$ System. Although, in many cases, the parameters of $1 \mathrm{D}$ systems can be calculated exactly, their complete theoretical description has not yet been accomplished. 1D systems belong to the class of strongly fluctuating systems [59]; thus, in contrast to systems of higher dimensionalities, they cannot unambiguously be described only by mean values such as mean density and compressibility [59]. The transport properties of $1 \mathrm{D}$ systems can also be rather specific (see $[43,57,60]$ and Section 2.1). Recent ab initio calculations [61] have shown that water flux through hydrophobic nanotubes is more than one order of magnitude higher than the estimated ordinary diffusion flux. The permeation experiments on zeolite membranes [57] have shown that for gases, whose particle diameter is larger than half the zeolite channel diameter, the increase of the diffusion coefficient with density is more than one order of magnitude larger than would be expected for one-particle diffusion. On the other hand, there are also experimental results, for example, obtained using nuclear magnetic resonance (NMR) and quasi-elastic neutron scattering (QENS) techniques, indicating that the particle mobility in $1 \mathrm{D}$ systems can decrease and the mean square displacement (MSD) can become proportional not to time, as it is in the case of a normal (Einstein) diffusion, but to the square root of time, $\left\langle x^{2}\right\rangle \sim t^{1 / 2}$ (so-called single-file diffusion mode (SFD)) $[43,62-67]$. Moreover, the experiments [64, 66] show that both the Einstein and SFD diffusion modes can exist for the same particles but at different densities and observation times. Furthermore, for certain investigated gases, a density increase leads to a transition from the Einstein diffusion to the SFD mode [64]. Thus some experiments suggest that the particle mobility in 1D systems must decrease with increasing density, whereas others show that diffusion accelerates as density becomes larger. An explanation for this contradiction is absent at present.

Diffusion in 1D systems has been studied in numerous theoretical works $[36,57,67-73]$. The dependence $\left\langle x^{2}\right\rangle \sim t^{1 / 2}$ has been obtained for a $1 \mathrm{D}$ system within the screened-gas model, which partly takes into account correlations between the particles [68-73]. It has been suggested [70-73] that the appearance of the two diffusion modes, characterized by the different time dependences of MSD, can be explained by a transition from the one-particle diffusion mode, occurring at short times, to SFD appearing at longer times when the particle mobility is limited by its neighbors. In this case at $t \rightarrow \infty$ the dependence of MSD is always $\left\langle x^{2}\right\rangle \sim$ $t^{1 / 2}$. Although this approach can describe the experimentally observed dependences of MSD, corresponding to SFD, it fails to explain the increase of the diffusion coefficient and transport acceleration observed in dense $1 \mathrm{D}$ systems [43]. The physical reason for this failing is that the proposed model [60] considered the system of individual tagged particles, whereas in experiments one does not follow the diffusion of a particular particle but rather deals with the system 
of many of identical particles. We propose a description for the particle diffusion [57] and mobility [43, 62-65] in $1 \mathrm{D}$ systems of identical particles with blocking effect (BE) when the motion of a particle is blocked by other particles or clusters. According to our model, different mobility and diffusion transport mechanisms can be realized in a $1 D$ system depending on its density. At low densities, an ordinary diffusion of single particles takes place. As density increases, a formation of $1 \mathrm{D}$ clusters of a finite size and lifetime takes place. In this case, there are two competing mechanisms prevalent in the system: a barrier-free propagation of density perturbations ("collective diffusion" (CD)) [60] and a blocking effect. When BE dominates, the SFD mode is realized, whereas when the $\mathrm{CD}$ mechanism prevails, we predict an essential transport acceleration, as compared to the oneparticle diffusion mode. In this case, MSD is Einstein-like with the diffusion coefficient increasing with density. The proposed model consequently takes into account all the timespace correlations occurring in a $1 \mathrm{D}$ system composed of identical particles with an arbitrary interaction potential. This model is able to describe the experimentally observed MSD dependencies at different densities as well as the transport acceleration with increasing density of a $1 \mathrm{D}$ system. To describe the transport properties of a $1 \mathrm{D}$ system, we apply the response function method (see Section 2.1 and [31, 32, 48]). This approach allows one to consistently take into account many-body correlations and obtain the diffusion coefficient and MSD for a 1D system of arbitrary density. Within response function method, we calculate the response function $\beta(k, \omega)$, which determines the Fourier image of the density fluctuation $\delta n(k, \omega)$ occurring in the $1 \mathrm{D}$ system in the external field of frequency $\omega$ and wave number $k$. The relaxation spectrum of a density fluctuation $(\omega(k))$ can be calculated from the relation $\beta^{-1}(k, \omega)=0$. Note that in response function method, $\delta n(x, t)$ can be interpreted as a distribution function if $t \gg a^{2} / D$, where $a$ is the particle diameter and $D$ is the one-particle diffusion coefficient [48]. A density fluctuation $\delta n(x, t)$ in a 1D system is determined by the relation (87), and the MSD can be expressed as

$$
\left\langle x^{2}\right\rangle=\frac{\int x^{2} d x d k e^{-i \omega(k) t} e^{i k x}}{\int d x d k e^{-i \omega(k) t} e^{i k x}} .
$$

It is evident from (87) and (83) that MSD is determined by the relaxation spectrum $\omega(k)$. The evaluation of $\omega(k)$ is reduced to the solution of Dyson equation for the response function (55) and for the 1D system takes the form (70), where $\nu(k, \theta)$ is the Fourier image of the pair correlation function of interacting particles. The function $\nu(k, \theta)$ can be obtained for a $1 D$ system with an arbitrary interaction potential $\Phi(x)[45]$ :

$$
\begin{gathered}
v(k, \theta)=\frac{2}{n_{0}}\left(\frac{\phi(p(\theta)-i k a)}{\phi(p(\theta))-\phi(p(\theta)-i k a)}+\frac{\theta}{i k a}\right), \\
\phi(z)=\int_{0}^{\infty} e^{-z x} \exp \left(-\frac{\Phi(x)}{T}\right), \\
\frac{1}{\theta}+(\ln \phi(z))_{z=p(\theta)}^{\prime}=0 .
\end{gathered}
$$

Here $T$ is the temperature. The last expression in (84) helps us to obtain the dependence of pressure on the filling factor $(p(\theta))$. It follows from $(70)$ and $(84)$ that at $\theta \rightarrow 0$, the relaxation spectrum $\omega(k)$ describes the one-particle diffusion $\omega(k)=-i D_{0} k^{2}$. For $k a \gg 1$, the Fourier image of the pair correlation function $\nu(k, \theta)$ approaches zero for any arbitrary short-range potential $\Phi(x)$. This corresponds to the absence of correlations between the particles at the length scale $a<l_{0}$, where $l_{0}$ is the average particle path between two collisions. In this case, the relaxation spectrum $\omega(k)$ also describes the one-particle diffusion mode $\omega(k)=-i D_{0} k^{2}$. The analysis of the relaxation spectrum performed in Section 2.1 for the hard-core potential has shown that due to collective effects and density fluctuations, the $1 \mathrm{D}$ system can transform into a nonhomogeneous state as density increases. This state is characterized by the formation of $1 \mathrm{D}$ clusters with a finite lifetime (Section 2.3) (Section 2.1). In this case at high density $(\theta \rightarrow 1)$, long distances $(k \rightarrow 0)$, and $t \rightarrow \infty$ (see $(70)$, (84)) the spectrum becomes diffusion-like with the diffusion coefficient increasing with density (72) that corresponds to the $\mathrm{CD}$ mode existing in a nonhomogeneous, clustered $1 \mathrm{D}$ system [62]. In order to describe the relaxation of density fluctuations at times $t \gg a^{2} / D_{0}$ (that corresponds to the longtime limit $t \rightarrow \infty$ ) for an arbitrary filling factor $\theta$, one should do series expansions of (70) and (84) with respect to the parameter $k a \ll 1$ and keep the expansion terms following the first one. It is convenient to rewrite this expansion with respect to parameter $k l_{0}$. Having done this, the relaxation spectrum for $k \ll 1 / a$ can be written as

$$
\omega(k)=-i\left(D(\theta) k^{2}+F(\theta) k^{2}\left(k l_{0}\right)^{2}+i F_{1}(\theta) k^{2}\left(k l_{0}\right)\right),
$$

where the expansion coefficients are $D(\theta)=D_{0} \zeta(0, \theta), F_{1}(\theta)$ $=D \theta v^{\prime}(0, \theta) \zeta^{2}(0, \theta)$, and $F(\theta)=D \theta^{2}\left((1 / 2) \nu^{\prime \prime}(0, \theta) \zeta^{2}(0, \theta)-\right.$ $\left.\nu^{\prime 2}(0, \theta) \zeta^{3}(0, \theta)\right)$. The values with apostrophes represent the corresponding derivatives of $\nu(k, \theta)$ with respect to wave number $k$, calculated at $k=0$. Equations (83) and (85) can be used to evaluate the time dependence of $\left\langle x^{2}\right\rangle$ at any length scale, starting from a diffusion path of just few particle jumps up to those comparable to the total length of the channel. Note that the real part of the spectrum $\operatorname{Re} \omega(k)$, which at $\theta \rightarrow 1$ corresponds to the sound mode, describes the collective effect of density perturbation propagation $\left(\delta n(x, t) \sim \int d k e^{-i \omega(k) t} e^{i k x}=\int d k \delta n_{1}(k, t) \delta n_{2}(k, t) e^{i k x}, \delta n_{1} \sim\right.$ $\left.e^{i \operatorname{Re} \omega(t) t}\right)$, and its imaginary part $\operatorname{Im} \omega(k)$ determines the diffusion mode for the relaxation of a density fluctuation taking into account the length-time correlations between the particles $\left(\delta n_{2} \sim e^{-\operatorname{Im} \omega(k) t}\right)$. Equations (87), (83), and (85) allow us to study the mobility and distribution function of the particles in a 1D system for different length/time scales, filling factors and interaction potentials $\Phi(x)$. Figure 6 presents the dependences of the distribution function on the dimensionless coordinate $\bar{x}=x / l_{0}$, calculated for different dimensionless times $t^{\prime}=t / \tau\left(\tau \sim l_{0}^{2} / D_{0}\right)$ using the squarewell interaction potential with the well depth $V \sim T(u=$ $V / T \sim 1)$ and width $R$.

It is important to mention that the obtained results do not depend on the specific form of the pair interaction potential 


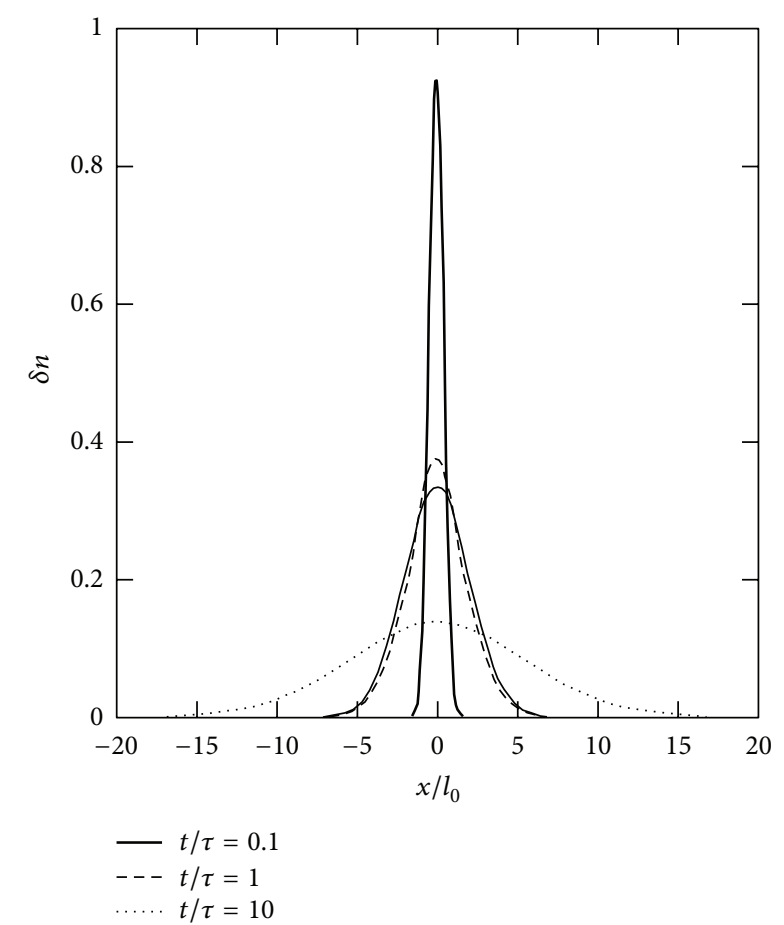

Figure 6: Typical distribution functions $\delta n(x, t)$ versus dimensionless coordinate $x / l_{0}$ (solid lines) at different times $t / \tau,\left(\tau \sim l_{0}^{2} / D_{0}\right)$ at $\theta \sim 0.2, u=1$, and $R=a$. Dashed lines show Gauss distributions for the corresponding times.

$\Phi(x)$; we have obtained similar results using rectangular potentials, the Lennard-Jones and Morse potentials. We also note that the shape of the distribution function is independent on density as both dimensionless coordinate and time are determined by the filling factor. The distribution functions at different time scales clearly differ from each other. The distribution is a Gauss-like function at short times $\left(\theta^{2} \ll t^{\prime}<1, \theta \ll|\bar{x}|<1\right)$ that corresponds to the one-particle diffusion (Figure 6). At $t^{\prime} \sim 1,|\bar{x}| \sim 1$, the distribution function varies faster than the Gauss distribution (Figure 6) that corresponds to limited mobility of a particle due to its blocking by other particles. At longer times $\left(t^{\prime} \gg 1\right.$, $|\bar{x}| \gg 1)$, the distribution function becomes Gauss-like again (Figure 6) as the transport in a dense 1D system is due to the $\mathrm{CD}$ mechanism [60]. In this case, the relaxation spectrum is diffusion-like with a density-dependent diffusion coefficient $D_{u}(\theta) \approx D_{0} /(1-\theta)^{2}$.

MSDs calculated at different time and length scales (Figure 7) using the distribution functions (Figure 6) noticeably differ from each other confirming the existence of several diffusion modes in the system. At short length-time scales $\left(t<\tau,\left\langle x^{2}\right\rangle \ll l_{0}^{2}\right)$, one should observe the one-particle diffusion mode with $\left\langle x^{2}\right\rangle \sim t$ (Figure 7(a)). When $\left\langle x^{2}\right\rangle$ is comparable to $l_{0}^{2}$ and time $t \sim \tau$, the correlations between the particles become essential that lead to the appearance of blocking effect. In this case, MSD has a non-Einstein character (Figure 7(b)). At longer times $t \gg \tau,\left\langle x^{2}\right\rangle \gg l_{0}^{2}$, the particle mobility is mostly determined by the second term in (85). This leads to the reappearance of the Einstein-type 110 dependence of MSD, $\left\langle x^{2}\right\rangle=2 D(\theta) t$, where $D(\theta)$ is an effective diffusion coefficient. Notice that the characteristic times at which different diffusion modes can be observed depend on the density of the system. Indeed, the average distance between the particles and, consequently, the time $\tau \sim 1 / \theta^{2}$ decrease with increasing density.

Further we compare our results to those of the QENS, NMR [43, 62-65], and permeation [57] experiments as well as to the results of the molecular dynamics (MD) calculations [71] summarized in Table 1 . Notice that such a system can be considered as one dimensional with the presence of $\mathrm{BE}$ as the diameters of the experimentally investigated molecules are less than the doubled diameter of the membrane channel. Our estimates for zeolite ZSM-48 obtained using the data from [71] show that for the low filling factor $(\theta=0.11)$ and times $\sim 10 \mathrm{~ns}$, the normal diffusion mode, $\left\langle x^{2}\right\rangle \sim t$, should occur while at higher density $(\theta=0.48)$ the dependence $\left\langle x^{2}\right\rangle \sim t^{1 / 2}$ should be observed, which is in a perfect agreement with the experimental data (Table 1). In Table 1 , experimental and MD results are presented; $a$ and $d$ are the particle and membrane channel diameters, respectively, $T$ is a temperature, and $\theta$ is a degree of filling of the channel. The fifth column represents the data on the motion mode of a particle in a channel ("SFD" means $x^{2} \sim t^{\alpha}, \alpha<1$, "normal" corresponds to $x^{2} \sim t$ ). The observation time of corresponding modes is represented in the sixth column.

The experimental results obtained for ethane molecules in zeolite $\mathrm{AlPO}_{4}-5$ and for methane molecules in zeolite ZSM48 (Table 1) are successfully described by our model. Indeed, using (85), one can write MSD for the SFD mode at $F(\theta)>0$ as

$$
\begin{gathered}
\left\langle x^{2}\right\rangle=(1-\theta) l_{0} \sqrt{32 D t F(\theta)} \\
F(\theta)=D_{0} \theta^{2}\left(\frac{1}{2} \nu^{\prime \prime}(0, \theta) \zeta^{2}(0, \theta)-\nu^{\prime 2}(0, \theta) \zeta^{3}(0, \theta)\right) .
\end{gathered}
$$

Equation (86) with $F(\theta)=1$ is identical to the relation derived in [62] with the accuracy of the numeric coefficient $\sqrt{32}$. Note that $F(\theta)$ describes the correlations effects in a $1 D$ system of interacting particles. Using (86) and the parameters for $\mathrm{AlPO}_{4}-5$ [62], we obtain $\left\langle x^{2}\right\rangle \sim 3 \mu \mathrm{m}$ for times $\sim 100 \mathrm{~ms}$ that is in good agreement with the values reported in $[62,64]$. At short times $t \leq l_{0}^{2} / D \sim 10^{-8}$ s, the main contribution comes from the first term in (85), and, in this case, the normal diffusion mode is realized. Our results obtained using (83) and (85) at $u=1$ and the experimental data for ethane molecules in zeolite $\mathrm{AlPO}_{4}-5$ are compared in Figure 8.

The dependence of the diffusion coefficient on the filling factor was investigated for several gases $\left(\mathrm{CH}_{4}, \mathrm{C}_{2} \mathrm{H}_{6}\right.$, and $\mathrm{C}_{3} \mathrm{H}_{8}$ ) in silicalite- 1 membrane at times $t>10 \mathrm{~s}$ [57]. The diffusion coefficients for these gases were found to increase more than 20 times as the filling factor varied from 0 to 0.9. This observation can be explained by the collective effect of density perturbation propagation through a $1 D$ clustered system. The calculated dependence of the relative diffusion coefficient $D / D_{0}=\left(\operatorname{Im} \omega(k) / D_{0} k^{2}\right)_{k=0}$ on the 

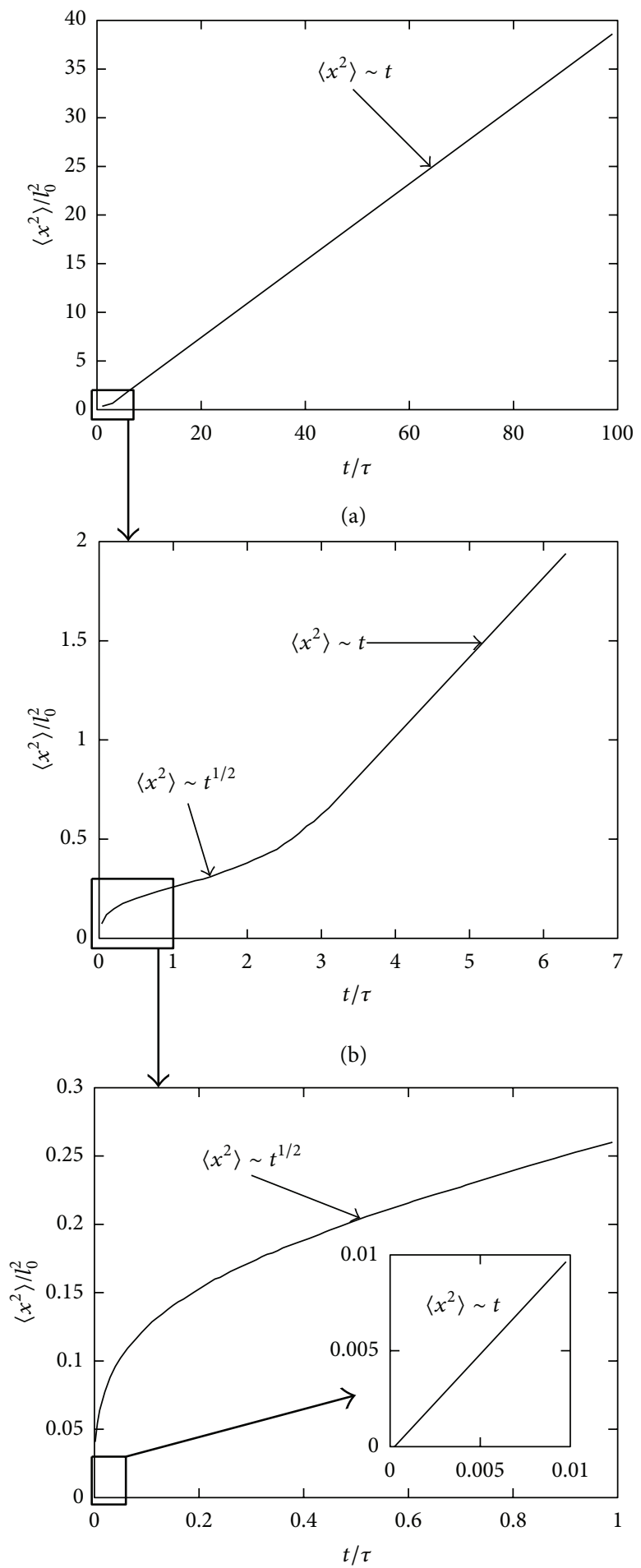

(c)

FIgURE 7: Typical $\left\langle x^{2}\right\rangle / l_{0}^{2}$ versus time $\left(t / \tau, \tau \sim l_{0}^{2} / D\right)$ dependence calculated for $\theta \sim 0.1, u=1$, and $R=a$ and shown at different time scales. (a) shows the curve up to $100 t / \tau$; its magnification is shown in (b) $(0: 7 t / \tau)$ and (c) $(0: 1 t / \tau)$. The insert in (c) shows very short times up to 0.01 , when the normal diffusion mode is observed. 
TABLE 1: Experimental and MD results: $a$ and $d$ are the particle and membrane channel diameters, respectively.

\begin{tabular}{|c|c|c|c|c|c|c|}
\hline Method & Molecule, zeolite & $T, \mathrm{~K}$ & $\theta$ & Diffusion mode & Observation time & Reference \\
\hline \multirow{2}{*}{ NMR } & $\mathrm{C}_{2} \mathrm{H}_{6}, a=3.8 \AA$ & 300 & 0.15 & SFD & $100 \mathrm{~ms}$ & \multirow{2}{*}[62]{} \\
\hline & $\mathrm{AlPO}_{4}-5, d=7.3 \AA$ & & & & & \\
\hline \multirow{4}{*}{ NMR } & & 180 & 0.003 & SFD & $2 \mathrm{~ms}$ & \multirow{4}{*}[71]{} \\
\hline & $\mathrm{CF}_{4}, a=4.7 \AA$ & & 0.03 & SFD & $150 \mathrm{~ms}$ & \\
\hline & $\mathrm{AlPO}_{4}-5, d=7.3 \AA$ & & 0.11 & SFD & $50 \mathrm{~ms}$ & \\
\hline & & & 0.22 & SFD & $150 \mathrm{~ms}$ & \\
\hline MD & $\mathrm{CF}_{4}, a=4.7 \AA$ & 180 & 0.11 & SFD & $10^{-8} \mathrm{~s}$ & \\
\hline \multirow{7}{*}{ QENS } & $\mathrm{CH}_{4}, a=3.8 \AA$ & 155 & 0.3 & Normal & $10^{-8} \mathrm{~s}$ & \multirow{7}{*}[64]{} \\
\hline & $\mathrm{AlPO}_{4}-5, d=7.3 \AA$ & & 0.45 & Normal & $10^{-8} \mathrm{~s}$ & \\
\hline & \multirow{3}{*}{$\begin{array}{l}\mathrm{C}_{2} \mathrm{H}_{6}, a=3.8 \AA \\
\mathrm{AlPO}_{4}-5, d=7.3 \AA\end{array}$} & 250 & 0.36 & Normal & $10^{-8} \mathrm{~s}$ & \\
\hline & & & 0.6 & Normal & $10^{-8} \mathrm{~s}$ & \\
\hline & & & 0.72 & Normal & $10^{-8} \mathrm{~s}$ & \\
\hline & $\mathrm{CH}_{4}, a=3.8 \AA$ & 155 & 0.11 & Normal & $10^{-8} \mathrm{~s}$ & \\
\hline & $\mathrm{ZSM}-48, d=5.6 \AA$ & & 0.48 & SFD & $10^{-8} \mathrm{~s}$ & \\
\hline
\end{tabular}

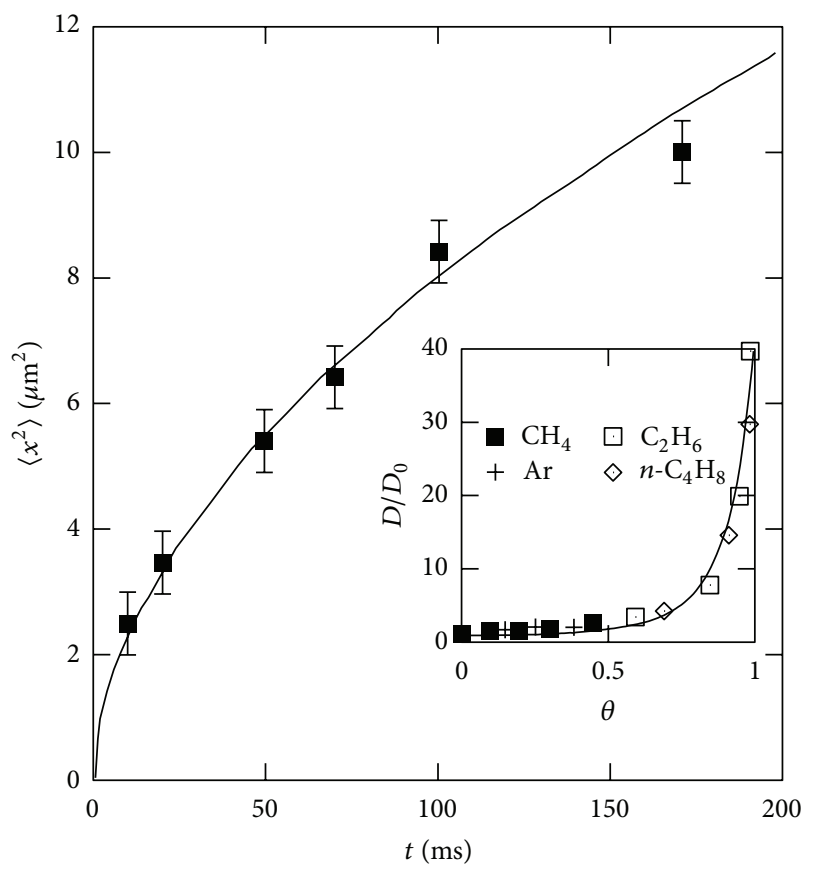

Figure 8: MSD versus time at $u=1, R=a$. The solid line shows the results calculated using (55), (70), (87), and (85); points represent the experimental data for the diffusion of ethane in zeolite $\mathrm{AlPO}_{4}-5$ [61]. The insert shows the dependence of the relative diffusion coefficient on the filling factor obtained for several gases (solid line) using (84) and (85) and $V / T=1$. Points are the experimental data from [57].

filling factor $\theta$ in comparison with experimental results is presented in Figure 8 (insert). The figure shows that the diffusion coefficient is only weakly dependent on the filling factor for $\theta<0.5$ (Figure 8 insert) but it rapidly increases when the filling factor becomes larger. This behavior of $D(\theta)$ can be easily understood as at low filling factors, the particles inside the membrane channel do not interact with each other, no clusters are formed, and particle motion is purely diffusive.
The increase of the diffusion coefficient for $\theta>0.5$ is due to the cluster formation inside the channel and activation of the CD mode (Section 2.1).

2.3. Formation of High- and Low-Density Clusters in a $1 D$ System. In recent experiments [74], the one-dimensional (1D) Au chains formed on the (110) NiAl surface have been investigated at low temperature $(T=12 \mathrm{~K})$. This surface exhibits $1 \mathrm{D}$ grooves made of the rows of $\mathrm{Ni}$ atoms, which suppress the transverse movements of Au adatoms allowing them to move only along the groove. The measured $\mathrm{Au}-\mathrm{Au}$ distances in such chains were about $2.9 \AA$ that is very close to those in bulk gold [74]. The properties of $1 \mathrm{D}$ Au chains were also investigated in experiments [8, 12, 75-77] where they were either pulled out of a gold surface by an STM tip or created in a process of thin film etching. Such chains were stable during the observation time and consisted of several gold atoms. The Au-Au distances in some of them appeared to be strikingly large, up to 3.5-4.0 $\AA$ [75-77], whereas in others much smaller Au-Au separations ( 2.5-2.9 $)$ were observed $[8,12,78-80]$. These results suggest that a $1 \mathrm{D}$ gold chain can exist in two different states characterized by different interatomic distances.

The ab initio calculations performed at zero temperature have shown that a $1 \mathrm{D}$ gold wire should break when the AuAu separations exceed $\sim 2.9 \AA$ [81-84]. Being in agreement with the experiments reporting the Au-Au distances of 2.5$2.9 \AA$, this result, however, does not explain the existence of large Au-Au separations. It has lately been demonstrated that the presence of hydrogen or other light atoms in the wire structure can stabilize gold chains and significantly alter their properties [3, 4, 82-88]. Assuming a somewhat ordered distribution of impurities within the wire, one can easily explain the experimentally observed large $\mathrm{Au}-\mathrm{Au}$ separations $[6,82,83,87]$. Although this finding offers an intriguing possibility to modify the wire properties by an intentional incorporation of impurities (Im), a spontaneous formation 
of locally ordered Au-Im-Au structures seems unlikely and remains to be proven experimentally. Another explanation has been proposed by Sun et al. [89] who suggested that the large $\mathrm{Au}-\mathrm{Au}$ distances might result from chain melting. It is known, however, that phase transitions do not occur in 1D systems [34]; thus, the proposed model should be taken with precaution.

Monoatomic chains can disintegrate due to atomic movements in both longitudinal and transverse directions caused by thermal fluctuations. The probability of disintegration is determined by the characteristic frequencies of atomic vibrations in the chain, and the energy barrier atoms need to be overcome in order to leave the chain, which is comparable with the energy per atom in the chain. A change of interatomic distances due to transversal or longitudinal motion of one of the atoms leads to a transition to a nonequilibrium state, and the energy per atom increases. As the energy barriers in the longitudinal and transversal directions are comparable [90], the disintegration probabilities for these two mechanisms appear to be comparable as well. Therefore, further on we consider only one mechanism occurring due to longitudinal fluctuations.

The probability of disintegration of a chain due to longitudinal fluctuations is determined by the frequency spectrum of relaxation of density fluctuations (relaxation spectrum) of the quasi-1D system. To study the relaxation spectrum of a quasi1D system, we apply the previously stated response functions for density functional method, which allows one to describe the equilibrium and dynamical properties of a system if its interaction potential is known. Within this method, one can find the response function $\beta(x, t)$ that determines the relation between the density fluctuation $\delta n(x, t)$ and the external field as well as all the space-time correlations in the system. Additionally, one can find the free energy of the quasi-1D system taking into account density fluctuations ([38] and Section 2.1). A density fluctuation appearing in the quasi-1D system can be determined as

$$
\begin{aligned}
& \delta n(x, t)=\operatorname{Re}\left(\int_{0}^{\infty} d k A(k) e^{-i \omega(k) t} e^{i k x}\right), \\
& A(k)= \begin{cases}A=\text { const, } & k_{\min } \leq k \leq k_{\max } \\
\sim 0, & \text { otherwise. }\end{cases}
\end{aligned}
$$

Here $k$ is the modulus of the wave number, and $A(k)$ is the amplitude of the density fluctuation, which in the analysis of cluster relaxation can be arbitrary and adequately considered to be a constant for $k$ in the interval $\left\{k_{\min }=2 \pi / L ; k_{\max }=\right.$ $2 \pi / d\}$. Clusters forming in the system are characterized by the parameter $\delta n(x, t)$, which is defined as a Fourier integral (87) in the interval $\left\{k_{\min } ; k_{\max }\right\}$, where $L$ is the cluster length, and $d$ is the atomic diameter. The cluster lifetime $(t)$ is determined by the maximal magnitude of the imaginary part of the spectrum $\omega(k)\left(\tau^{-1}=\operatorname{Im} \omega(k)\right)$ in the interval $k_{\min }<k<$ $k_{\max }$.

The relaxation spectrum of the 1D system $\omega(k)$ is determined by (70). If the relaxation of such a system is determined by diffusion with the coefficient $D_{0}$, then $\omega_{0}(k)=-i D_{0} k^{2}$. $\theta=n_{0} d$ is a dimensionless density. $n_{0}$ is the average number of particles per length unit. $\nu(k, \theta)$ is a Fourier image of the pair correlation function for interacting particles, which is related to the Fourier component of the pair distribution $(g(k))$ as $\nu(k)=g(k)-\delta(k)$, where $\delta(k)$ is the Dirac delta-function. Note that the divergence must be removed from $g(k)$ before any analysis. Otherwise reverse Fourier transformation and the transition from wave numbers to coordinates are impossible due to the integral divergence. For a quasi-1D system, the pair distribution can be calculated exactly for an arbitrary short-range interaction potential $\Phi(x)[44]$ :

$$
\begin{gathered}
g(x)=\frac{1}{2 \pi} \int_{0}^{\infty} d k e^{i k x} g(k), \\
g(k)=\frac{1}{n_{0}} \frac{\phi\left(p_{1 \mathrm{D}} / T-i k\right)}{\phi\left(p_{1 \mathrm{D}} / T\right)-\phi\left(p_{1 \mathrm{D}} / T-i k\right)}, \\
\phi\left(p_{1 \mathrm{D}}\right)=\int_{0}^{\infty} \exp \left(-\frac{p_{1 \mathrm{D}} x+\Phi}{T}\right) d x .
\end{gathered}
$$

Here $p_{1 \mathrm{D}}$ and $T$ are $1 \mathrm{D}$ pressure and temperature, respectively. Equation (88) allows one to evaluate the pair distribution and consequently the relaxation spectrum of the $1 \mathrm{D}$ system. We have performed an extensive analysis, which has shown that the properties of quasi-1D clusters are determined by the presence of the repulsive and attractive terms in the potential but not by the specific form of $\Phi(x)$. In particular, we have obtained similar results using rectangular potentials, and the Lennard-Jones as well as Morse potentials. We notice that this finding correlates well with the known results of the liquid state theory $[45,46,91]$. For the sake of simplicity, we present here the calculations of $1 \mathrm{D}$ pressure $p_{1 \mathrm{D}}(\theta)(\theta=(\mathrm{Nd} / L)$ is dimensionless density) and pair distribution done for the square-well potential with the well width $R$ and depth $U_{0}$ :

$$
\Phi(x)= \begin{cases}\infty, & x \leq d \\ -U_{0}, & d<x \leq d+R \\ 0, & x>d+R .\end{cases}
$$

The performed calculations give the $p_{1 \mathrm{D}}(\theta)$ dependence:

$$
p_{1 \mathrm{D}} d\left(\frac{1}{\theta}-1\right)=T-p_{1 \mathrm{D}} R\left[\frac{\exp \left(p_{1 \mathrm{D}} R / T\right)}{\left(1-\exp \left(U_{0} / T\right)\right)}-1\right]^{-1} \text {. }
$$

Equations (88) and (90) allow one to analyze the possible states of the quasi-1D system of the interacting particles of an arbitrary density. They also let us to estimate the relaxation time of a density fluctuation (cluster) as well as the interatomic distances in the clusters. We choose to perform this analysis using the parameters of the pair potential (89), $U_{0}=1.1 \mathrm{eV}, d=2.5 \AA$, and $R=2.5 \AA$, corresponding to the gold atoms [80]. For these parameters, we calculate the compressibility $\eta=-\left(\partial \theta / \partial p_{1 \mathrm{D}}\right)$ (Figure 9$)$, pair distribution (Figure 10), and dimensionless spectrum $\Omega \equiv \omega(k) / \omega_{0}(k)$ (Figure 11).

At low densities $(\theta<0.67)$, the compressibility increases with increasing density and it approaches its first maximum at $\theta=\theta_{c}=0.18$ (Figure 9). The compressibility has a 


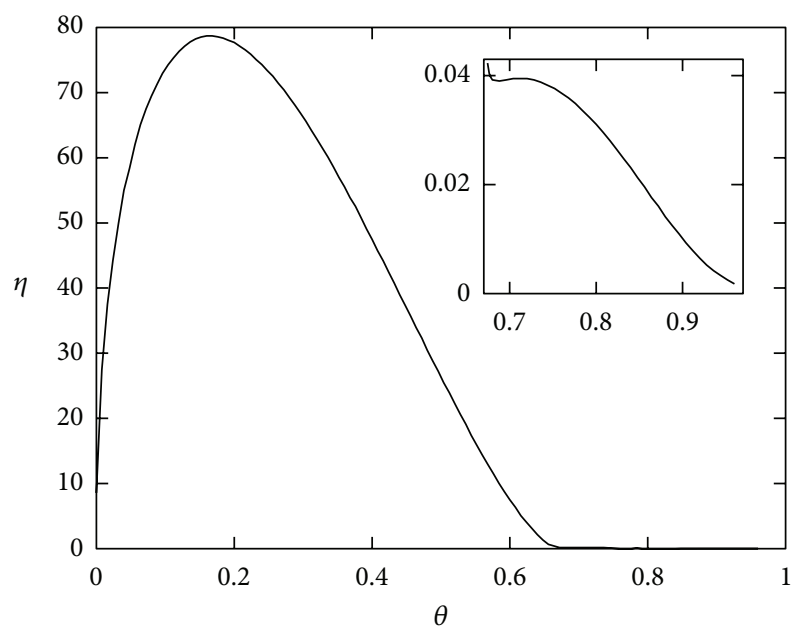

FIGURE 9: The compressibility versus density for $\mathrm{Au}$ atoms at room temperature.

second maximum at $\theta=\theta_{c}^{(1)}=0.71$. The derivative of the compressibility equals 0 at extrema. Therefore, the presence of extrema can indicate changes in the system state. As we show in the following, the presence of maxima does not depend on the specific form of the pair interaction potential $\Phi(x)$, but it is determined by the $U_{0} / T$ ratio $\left(U_{0}\right.$ is a characteristic depth of an arbitrary potential). The second maximum of the compressibility disappears if $U_{0} / T \ll 1$. When $U_{0} / T \gg 1$, the second maximum always exists if the condition $R \geq d$ is satisfied regardless of the specific form of the potential $\Phi(x)$. Also we have to note that the ratio $U_{0} / T$ provides the existence of the second maximum whereas the position of this maximum does not depend on the magnitude of this ratio. This allows us to conclude that in the limiting case $U_{0} / T \gg 1$, the variation of the temperature of a system does not lead to any qualitative changes in a state of a system.

To demonstrate how the physical state of the system changes at the compressibility maxima, let us analyze the pair distribution $g(x)$ that determines the probability to find atoms at a certain distance from each other. It has been shown in Section 2.1 that the function $g(x) \rightarrow 1$ when $x \rightarrow \infty$. The distances between the maxima of $g(x)$ are equal to $1,2,3$, and so forth, atomic diameters. Hence, the separation between these maxima corresponds to the interatomic distance in the clusters [45].

In the vicinity of the first compressibility maximum $\left(\theta_{c}=0.18\right)$, the distance between the peaks of $g(x)$ and, correspondingly, the interatomic distances in the clusters are $\sim 3.6 \AA$ (Figure 10(a)). At densities close to the second maximum, interatomic distance in the cluster $\leq 3 \AA$ (Figure 10(b)). As it was mentioned above in the limiting case $U_{0} / T \gg 1$, the variation of the temperature of a system does not lead to any changes in the state of this system. Therefore, at $T=300$ and $12 \mathrm{~K}$, the magnitude of the gold cluster interatomic distance will be the same.

As it has been noticed previously the qualitative shape and the distance between the peaks of the pair distribution function are independent of the concrete form of the interaction potential. The pair distributions $g(x)$ calculated for $\theta=0.15$ with square-well potential (solid line) and Morse potential for the Au atoms (dashed line) are compared in Figure 11.

Figure 11 demonstrates that the distances between the peaks are the same for both potentials, but the peaks for the Morse potential are higher compared to the ones obtained with the square-well potential. This difference is due to the different characteristic length scales of the Morse and squarewell potentials. The characteristic length scale of the Morse potential is larger than that of the square-well potential that leads to a larger atomic attraction at long distances. Note that in the case of the Morse potential $U_{0}$ in (89) is a potential well depth and $d+R$ is the characteristic length scale of the potential.

Further, we analyze the stability of the $1 \mathrm{D}$ clusters. The change of the free energy of the $1 \mathrm{D}$ system $(\Delta \Phi)$ due to the cluster disintegration determines the probability of such a disintegration [34]. $\Delta \Phi$ can be divided into the following contributions: $\Delta \Phi_{0}$ that is the change of the free energy due to the pair interactions in the cluster; $\Delta \Phi_{1}$ that is the change of the free energy coming from changes in the cluster electronic states; and the fluctuation part $\Delta \Phi_{f}$ that is an average fluctuation of the free energy of the $1 \mathrm{D}$ system caused by cluster disintegration calculated for the stationary state of the $1 \mathrm{D}$ system. Therefore, the probability of cluster disintegration can be written as

$$
\begin{aligned}
& W \propto W_{f} W_{i}, \quad W_{f} \propto e^{\Delta F_{f} / T}, \\
& W_{i} \propto e^{\Delta F_{i} / T}, \quad \Delta F_{i}=\Delta F_{0}+\Delta F_{1} .
\end{aligned}
$$

To estimate the cluster lifetime $\tau \sim W^{-1}$, we analyze the contributions to $\Delta \Phi$. Since there are no phase transitions in $1 \mathrm{D}$ systems, $\Delta \Phi_{f}$ is always positive and its magnitude is determined by the spectrum $\Omega(k)$ [46] (Figure 12):

$$
\Delta F_{f} \sim T \int_{0}^{\infty} d k \Omega(k)(\delta n(k))^{2} .
$$

It follows from (92) that $\Delta \Phi_{f}$, which is related to the probability of cluster disintegration due to the loss of one or several atoms, is determined by the maximal magnitude of $\Omega(k)$ in the interval $\left(k_{\min }=2 \pi / L ; k_{\max }=2 \pi / d\right)$. In the vicinity of the first maximum of the compressibility at $\theta=0.2$ (Figure 9), $\Delta \Phi_{f} \sim T N \Omega\left(k^{\prime}\right)$. Here $k^{\prime}$ is the wave number corresponding to the maximum of $\Omega(k), k^{\prime} d \sim 2$ (Figure 12(a)), and $N$ is the number of particles in the cluster. It follows from (91) that in this case the magnitude of $W_{f}$ is large for all the clusters with the length exceeding several atomic radii. In the vicinity of the second maximum of the compressibility at $\theta=0.7$ (Figure 9 ), $\Delta \Phi_{f}$ becomes larger as the cluster length increases (Figure 12(b)). In this case for all clusters with the length exceeding $L^{\prime}$, for which $\Omega\left(2 \pi / L^{\prime}\right) \gg$ $1, \Delta \Phi_{f} \sim T N \Omega\left(2 \pi / L^{\prime}\right)$ and the probability $W_{f}$ is large. Thus at all densities, only clusters of a certain maximal length can exhibit a noticeable lifetime, and the formation of long clusters appears to be unfavorable.

$\Delta \Phi_{i}$ is the sum of the changes of the free energy of the pair interaction between atoms in the cluster $\varepsilon_{0}$ and the free energy of the cluster electronic states $\varepsilon_{1}\left(\Delta \Phi_{i} / N=\varepsilon_{0}+\varepsilon_{1}\right)$. 


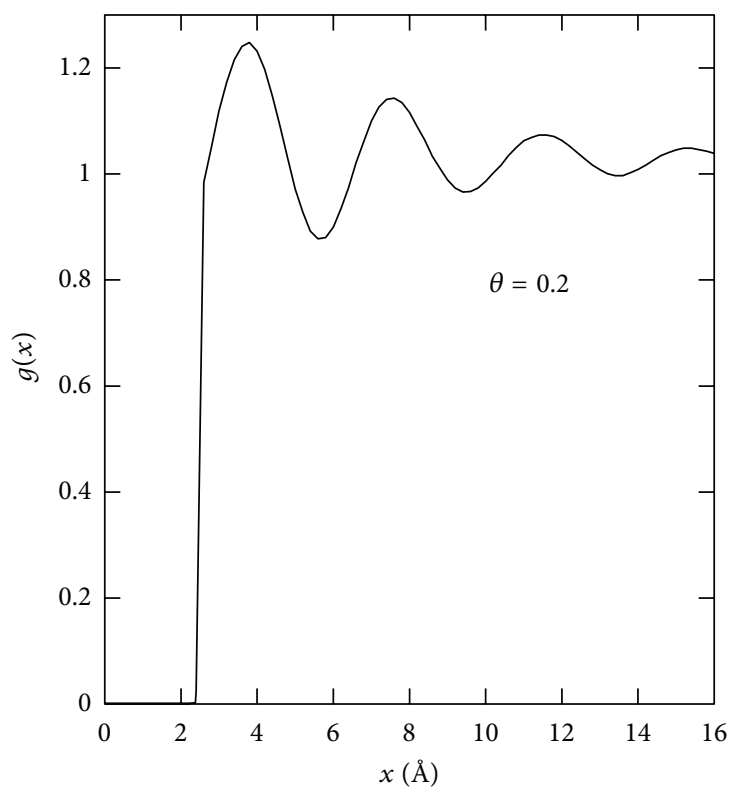

(a)

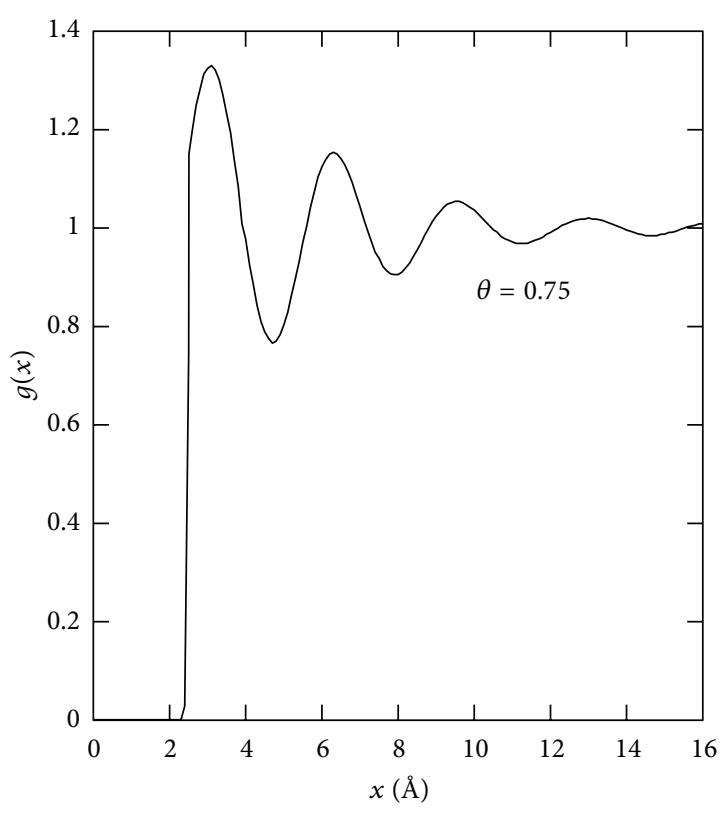

(b)

Figure 10: Pair distribution of gold atoms at room temperature.

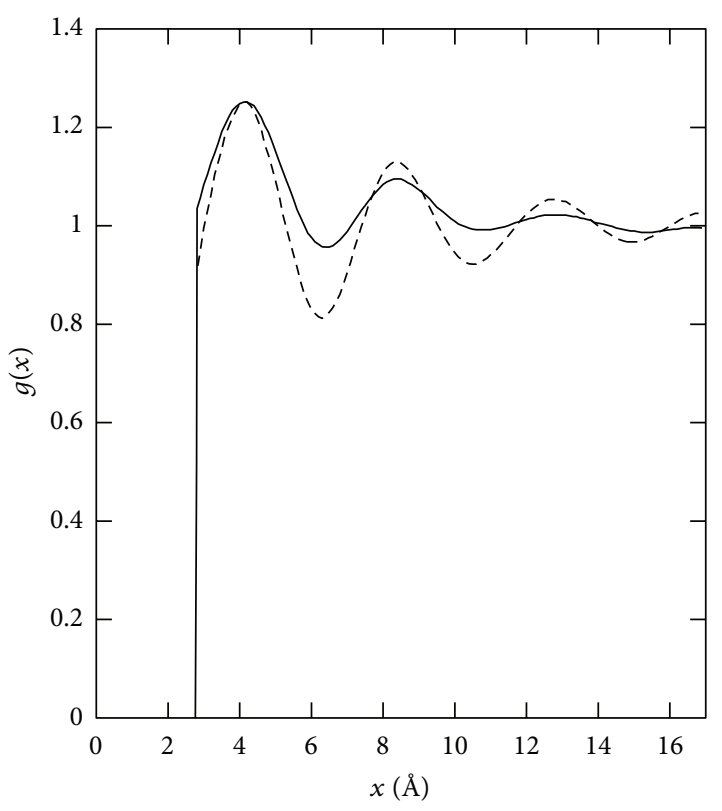

FIGURE 11: Pair distribution calculated with both square-well (solid) and Morse (dashed) potentials.

In the limiting case $T \ll\left|\varepsilon_{1}\right|, \varepsilon_{1}$, is negative [92] and it is determined by the overlap of the atomic orbitals. This overlap is determined by the penetrability coefficient $\Gamma$ of the potential barrier separating the electronic potential wells of the neighboring atoms (Section 2.4). In the quasi-classical approximation, $\Gamma$ depends exponentially on the width and height of the potential barrier (Section 2.4). It has been shown [92] that due to collective effects, the penetrability coefficient
$\Gamma$ depends not only on the interatomic distances, but also on the difference between the maximum and minimum values $(\Delta g)$ of the pair distribution $g(x)$ (Figure 10). The interatomic distances in the clusters formed at the densities about $\theta \sim 0.2$ are larger than those for the clusters existing at $\theta \sim 0.7$ (Figure 10), but $\Delta g(\theta=0.75)>\Delta g(\theta=0.2)$. Using the approach of [92], we find that in this case the magnitudes $\left|\varepsilon_{1}\right|\left(\left|\varepsilon_{1}\right| \sim G\right)$ for the clusters of both kinds appear to be similar. Thus, for our analysis of cluster stability, we can use the value $\left|e \varepsilon_{1}\right| \sim 0.5 \mathrm{eV}$ obtained from the measurements of the cluster conductivity in the scanning tunneling spectroscopy (STS) experiments for the Au chains on the $\mathrm{NiAl}$ surface [74]. The energy $\varepsilon_{0}$ per atom can be written as $\varepsilon_{0} \sim(\theta / 2) \int_{0}^{\infty} d x \Phi(x) g(x)$ [46]. Calculations show that $\varepsilon_{0}$ is negative and $\left|\varepsilon_{0}\right| \sim 1 \mathrm{eV}$ and $\left|\varepsilon_{0}\right| \sim 0.7 \mathrm{eV}$ for the clusters at $\theta \sim 0.7$ and $\theta \sim 0.2$, respectively.

Let us now compare the lifetime and interatomic distances calculated for the 1D clusters to the known properties of the $1 \mathrm{D}$ chains fabricated in experiments [74-80]. The results of the STS experiments [74] as well as the ab initio study of the electronic structure of gold wires on (110) NiAl [93] suggest that the main role of the substrate is to be a natural template insuring the one-dimensionality of the $\mathrm{Au}$ chains. This allows one to suggest that the observed $\mathrm{Au}-\mathrm{Au}$ distances $(\sim 2.9 \AA)$ in such chains to a large extent might be determined by the one-dimensionality of the system. Taking into account the minimal possible distance between Au atoms $(\sim 2.5 \AA[81])$, we can estimate the density in these chains: $\theta=(2.5 / 2.9)=0.87$. Thus, the fabrication of Au chains on (110) NiAl can be understood as an artificial formation of $1 \mathrm{D}$ clusters with the density $\theta$ fulfilling the condition $\theta>$ $\theta_{c}^{(1)}\left(\theta_{c}^{(1)}=0.71\right)$. As we have shown previously, the AuAu separations in such $1 \mathrm{D}$ clusters should range from $3 \AA$ 


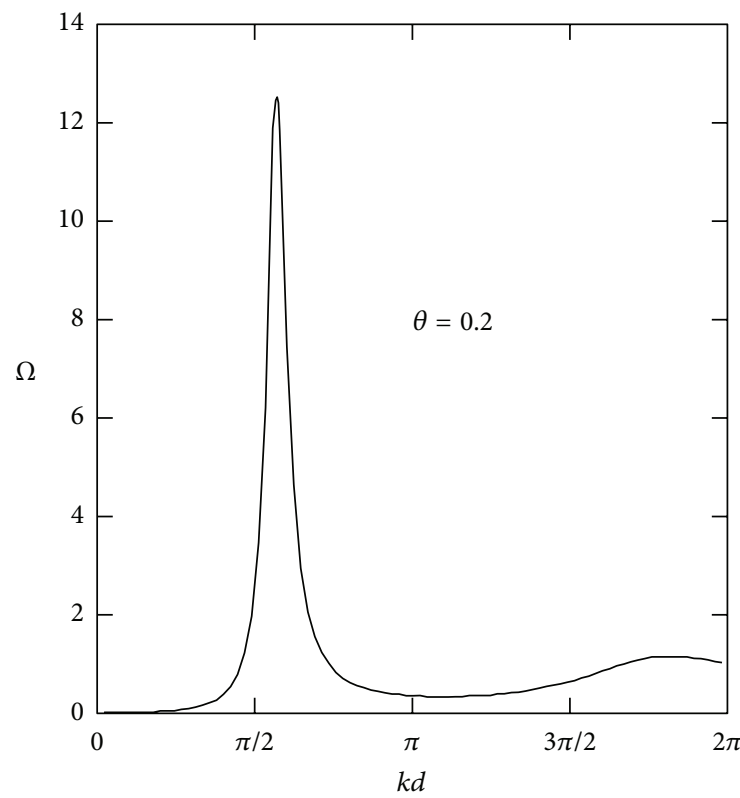

(a)

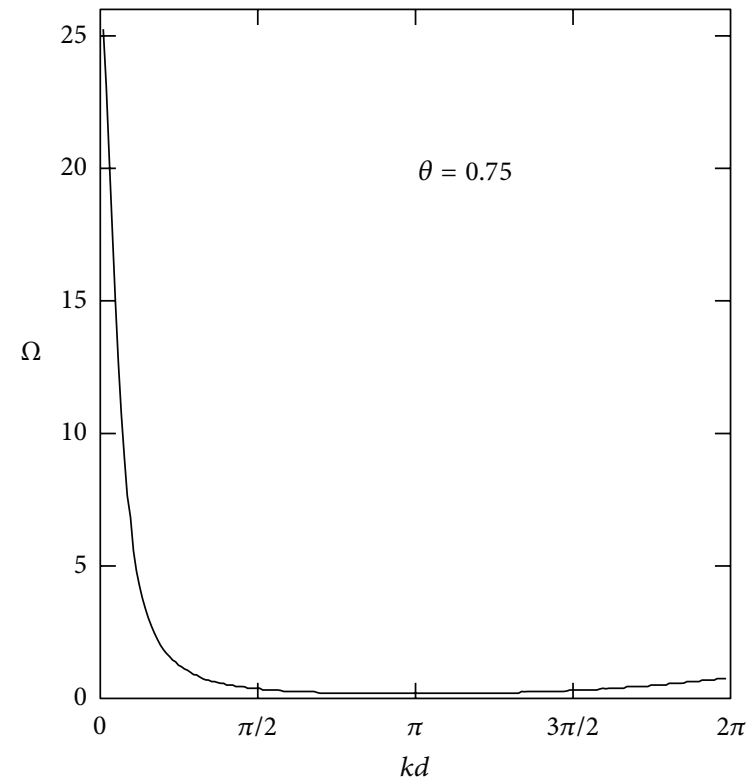

(b)

FIGURE 12: Dimensionless spectrum $\Omega(k)$ versus the wave number $k$.

$(\theta=0.74)$ to $2.7 \AA(\theta=0.9)$ or $2.5 \AA(\theta=1)$. The lifetime of such clusters of the length $L$ can be estimated as the characteristic time $\left(\tau \sim \tau_{f} \exp (Q / T)\right)$ during which an atom stays in the potential well of the depth $Q=\left|\varepsilon_{0}\right|+\left|\varepsilon_{1}\right|-$ $T \Omega(2 \pi / L)$ (see $(91))$. Here $\tau_{f} \sim \omega_{0}^{-1}(k=2 \pi / d) \sim 10^{-12} \mathrm{~s}$. The calculations of the spectrum $\Omega(k)$ at $\theta=0.87$ show that, for example, for the cluster of the length $L \sim 15 \AA$, containing $\sim 5 \mathrm{Au}$ atoms $\Omega(2 \pi / L) \sim 10$. In this case using the estimates obtained previously $\left(\left|\varepsilon_{1} \sim 0.5 \mathrm{eV},\right| \varepsilon_{1} \mid \sim 1 \mathrm{eV}\right)$, one can estimate the lifetime of such chains to be $t>1000 \mathrm{~s}$ $(T=300 \mathrm{~K})$. Notice that in the absence of collective effects and attractive interaction between atoms in a quasi-1D system the lifetime of the chain of $L \sim 15 \AA$ would be very short, $\tau \sim \omega^{-1}(k=2 \pi / L) \sim L^{2} / D \sim 10^{-9} \mathrm{~s}$ (here $D \sim 10^{-5} \mathrm{~s} \mathrm{~m}^{2} / \mathrm{s}$ is the diffusion coefficient for $\mathrm{Au}$ atoms at the surface of $\mathrm{NiAl}$ [94] at $T=300 \mathrm{~K})$.

The formation of suspended Au chains by pulling them out of the $\mathrm{Au}$ surface or by etching an $\mathrm{Au}$ film with an electron beam [2, 8, 12, 75-79] corresponds to conditions where considerable tensile stress is applied to the chain. The presence of an external force corresponds to the appearance of the one-dimensional pressure $p_{1 \mathrm{D}}=-(\partial F / \partial L)$, where $F$ is a free energy of the $1 \mathrm{D}$ chain. One can see from (90) that $1 \mathrm{D}$ chains of finite length are affected by two types of forces: forces corresponding to the effective repulsion of the atoms from each other as a result of their movement (the first term in (90)) and forces corresponding to their mutual attraction that is determined by interaction potential of atoms with each other. The attractive force $\left|p_{1 D}^{v}\right|$ depends on depth of potential well and radius of the potential $R$ and equals $p_{1 \mathrm{D}}^{v}=-\left(N^{2} / L^{2}\right) \varepsilon R$ in the van der Waals approximation. Thus a cluster is in a compressed state. The value of compression is $\left|p_{1 D}^{v}\right|=\left(N^{2} / L^{2}\right) \varepsilon R$. The presence of such a compression can lead to loss of stability of the cluster in the transverse direction and formation of a zigzag configuration of a chain. Therefore, to form a stable linear chain, it is necessary to attach an external force to the end of a chain to compensate the compression $\left|p_{1 \mathrm{D}}^{v}\right|$. The presence of an external force corresponds to the appearance of one-dimensional pressure $\left|p_{1 \mathrm{D}}^{v}\right|=-(\partial F / \partial L)$, where $F$ is the free energy of a onedimensional chain. Let us estimate the lifetime of these chains.

The decrease of the tensile force corresponds to the decrease of the density $\theta$ (90). The observation of Au chains [75-77] containing three or more atoms and exhibiting large $\mathrm{Au}$-Au separations $(\sim 3.6 \AA)$ can be considered as a formation of a $1 \mathrm{D}$ cluster at the pressure $p_{1 \mathrm{D}}$ for which $y$ satisfies the condition $\theta_{c}<\theta<\theta_{c}^{(1)}\left(\theta_{c}=0.18, \theta_{c}^{(1)}=0.71\right)$. The lifetime of such chains can be estimated as $\tau \sim \tau_{f} \exp (Q / T)$, where $Q=\left|\varepsilon_{0}\right|+\left|\varepsilon_{1}\right|-T \Omega\left(k^{\prime}\right), k^{\prime} d \sim 2$. For $\left|\varepsilon_{1}\right| \sim 0.7 \mathrm{eV},\left|\varepsilon_{0}\right| \sim 0.5 \mathrm{eV}$ and $\Omega\left(k^{\prime}\right) \sim 10$ (Figure 12(a)), it turns out that $\tau>100 \mathrm{~s}$ $(T=300 \mathrm{~K})$. The experimentally obtained chains $[1,8,12,78-$ 80] with the Au-Au separations of 2.5-2.9 $\AA$ are formed at the pressure $p$ for which $\theta$ fulfills the condition $\theta>\theta_{c}^{(1)}$. According to our estimations, the Au-Au separations in such clusters should be $<3 \AA$ (Figures 10 (b) and 12(b)) and their lifetime is $\tau>1000 \mathrm{~s}(T=300 \mathrm{~K}, L \sim 25 \AA, \Omega(2 \pi / L) \sim 20)$ (Figure 11).

Within the framework of these assumptions, we can also estimate minimal lifetime of chains of other metals, such as $\mathrm{Ag}, \mathrm{Ni}$, and Pt. Due to the lack of experimental data on the characteristic values of $\varepsilon_{0}$ for these materials let us assume $\left|\varepsilon_{1}\right|+\left|\varepsilon_{2}\right| \sim|\varepsilon|$, where $\varepsilon$ is the binding energy per atom for dimer [95]. The calculated values of minimal lifetime of one-dimensional chains are presented in Table 2. As one can 
TABLE 2: Minimal chain lifetime $\tau$, minimum number of atoms in a stable chain $N_{\min }$, and $F_{0 \text { th }}, F_{0 e}$ are the estimated and experimental values of the force required to break the cluster with a minimum number of atoms in it correspondingly.

\begin{tabular}{lcccc}
\hline & $\mathrm{Ag}$ & $\mathrm{Au}$ & $\mathrm{Ni}$ & $\mathrm{Pt}$ \\
\hline$|\varepsilon|, \mathrm{eV}[95]$ & 1.15 & 1.46 & 1.78 & 2.22 \\
$a, \AA[95]$ & 2.58 & 2.58 & 2.08 & 2.32 \\
$\tau, \mathrm{s}$ & $>1$ & $>10^{2}$ & $>10^{5}$ & $>10^{7}$ \\
$N_{\text {min }}$ & 2 & 3 & 4 & 5 \\
$F_{0 \text { th }}, \mathrm{nN}$ & 1.4 & 2.03 & 1.5 & 2.2 \\
$F_{0 e}, \mathrm{nN}[13]$ & 1.2 & 0.19 & 1.7 & 3.1 \\
\hline
\end{tabular}

see, the material of a one-dimensional chain greatly affects its stability.

Let us estimate the minimal length of stable chains and the force that is necessary for their formation. Note that if we consider the chain as a rod of length $L$ with a radius equal to the atomic radius $\sigma$, then such a rod loses its stability when it is compressed with a force greater than $F_{c}=\pi^{3} E \sigma^{4} / L^{2}$ [96], where $E$ is the Youngs modulus. So, if the compression pressure of the chain is $\left|p_{1 \mathrm{D}}^{v}\right|>F_{c}$, then the chain is unstable. Therefore, the minimum compressive force that ensures stability of the chain is $\left|p_{1 D}^{v}\right|=F_{c}$. Chains of metal atoms have a conductivity comparable to the metal one [13], so the range of the interatomic potential is comparable to the length of the chain $R=\kappa L$, where $\kappa \sim 1$ and varies from metal to metal. Relations $\left|p_{1 \mathrm{D}}^{v}\right|=\left(N^{2} / L^{2}\right) \varepsilon R$ and $F_{c}=\left(p i^{3} E \sigma^{4} / L^{2}\right)$ allow to estimate the minimum number of atoms in a cluster $N_{\min }=\pi\left(E \sigma^{4} / \kappa a\right)^{1 / 3}$, where $a$ is an interatomic distance in the cluster. The value $\left|p_{1 \mathrm{D}}^{v}\right|$ is the force that compresses a stable cluster without external influences on it. In this case, $\left|p_{1 \mathrm{D}}^{v}\right|=\left(N_{\min } / a\right) \varepsilon \kappa$ is a force $F_{0}$ required to break the cluster with the minimum number of atoms in it. Table 2 shows the results of the estimations of the minimum number of particles in the chain $N_{\min }$ and the corresponding force required to break the chains $F_{0 \text { th }}$ for chains of different material. It was assumed that $\kappa_{\mathrm{Ag}}=1$ for the chains of Ag. For the rest of metal chains, this coefficient was estimated by using the relative conductivities $\kappa_{i}=\kappa_{\mathrm{Ag}}\left(\sigma_{i} / \sigma_{\mathrm{Ag}}\right), i=\mathrm{Au}, \mathrm{Ni}, \mathrm{Pt}$.

\subsection{Transport of a Two-Component Mixture in One-Dimen-} sional Channels. The dependence of transport selectivity on the pressure in a two-component system of atomic particles is nonmonotonic: the selectivity attains its maximal value and then decreases instead of increasing. Thus, the mechanism of diffusion enhancement in $1 \mathrm{D}$ channel, which was proposed in Section 2.1, is inapplicable for two-component mixtures.

The transport of a two-component mixture in subnanometer channels was considered earlier [51, 97] on the basis of the generalized phenomenological Stephan-Maxwell equation. The authors of these publications used the dependence of the chemical potential on the fill factor, taking into account the finite size of particles, but disregarding their interaction. The dependences obtained in [51, 97] also indicate a monotonous increase in the diffusion coefficients, fluxes, and selectivity upon an increase in $\theta$ and, hence, fail to describe the experimental data for two-component mixtures.

2.4.1. Ground State of a System in 1D Channels. Let us consider the surface of a porous body in contact with a twocomponent gas mixture at temperature $T$ and pressure $p$. Suppose that $N$ adsorption centers are located on the surface. We assume that the particles on the outer surface do not interact with one another. We also assume that the energy of a gas molecule on the surface is equal to $\varepsilon_{0}^{(i)}, i=1,2$, depending on the species of the molecule. We also suppose that $\kappa$ cylindrical channels $(\kappa \gg 1)$ of diameter $d$ and length $L$ emerge at the surface. We assume that the diameter of a cylinder is comparable to the maximal diameter of the gas molecule. Let us assume that $\varepsilon_{1}^{(i)}$ is the binding energy of the $i$ th particle at the mouth of a channel, $N_{1}^{(i)}$ is the number of particles of the $i$ th species above the membrane, $q^{(i)}$ is the total number of particles of the $i$ th species in the channel, $n^{(i)}$ is the total number of $i$ th particles in the channel and on the surface, and $N_{0}$ is the number of "seats" in the channel. Then the partition function of the grand canonical ensemble taking into account the interaction of system of atomic particles in the channel has the form

$$
\begin{aligned}
& \Theta= \sum_{i=1}^{2} \sum \frac{(N-k) ! \exp \left[\beta \varepsilon_{0}^{(i)}\left(N_{1}^{(i)}-n^{(i)}\right)\right]}{\left(N_{1}^{(i)}-n^{(i)}\right) !\left[n-k-\left(N_{1}^{(i)}-n^{(i)}\right)\right] !} \\
& \times \frac{\kappa ! \exp \left[\beta \varepsilon_{1}^{(i)}\left(n^{(1)}\right)-q^{(1)}\right]}{\left(n^{(1)}-q^{(1)}\right) !\left[\kappa-\left(n^{(1)}-q^{(1)}\right)\right] !} \\
& \times \frac{N_{0} ! \exp \left(\beta \varepsilon_{2}^{(i)} q^{(i)}\right)}{q^{(i)} !\left(N_{0}-q^{(i)}\right) !} \exp \left(\beta \mu N_{1}^{(i)}\right) Z_{\mathrm{int}}\left(q^{(1)}, q^{(2)}\right), \\
& N_{1}^{(i)}+q^{(i)}=n^{(i)} .
\end{aligned}
$$

Here, the second sum corresponds to summation over configurations $\beta=T^{(1)}, \varepsilon_{2}^{(i)}$ is the binding energy of particles of the $i$ th component in the channel, $\mu$ is the chemical potential, and $Z_{\text {int }}\left(q^{(1)}, q^{(2)}\right)$ is the partition function corresponding to the inclusion of particle interaction in the channel. Considering that the main contribution to the partition function comes from states with a large number of particles $\left(q^{(i)} \gg 1\right)$, we can replace partition function $Z_{\text {int }}\left(q^{(1)}, q^{(2)}\right)$ by the partition function for particles interacting in the channel, calculated for the mean value $\bar{q}^{(i)}$ of the number of particles of each species in the channel. From the physics standpoint, this corresponds to "averaging over channels," when a single channel (whose state is calculated by averaging the parameters of particles in $\kappa$ channels) is considered instead of the large number of channels. It should be noted that this approximation is possible since the number of channels having a diameter of $d<1 \mathrm{~nm}$ and emerging on a square centimeter of the zeolite membrane surface is large: $\kappa \sim l_{c}^{-2} \sim 10^{14} \mathrm{~cm}^{-2} \gg$ 1 , where $l_{c}$ is the characteristic distance between the channels on the membrane surface (Section 2.1). Using relation 
(93) and following the method proposed in Section 2.1, we obtain for the adsorption isotherm

$$
\begin{gathered}
\frac{a}{\sigma_{\mathrm{av}}} \theta=c \frac{p}{p+p_{0}^{(1)}}+(1-c) \frac{p}{p+p_{0}^{(2)}}, \\
\frac{a}{\sigma_{\mathrm{av}}} c_{1} \theta=c \frac{p}{p+p_{0}^{(1)}}, \quad \frac{a}{\sigma_{\mathrm{av}}} c_{2} \theta=(1-c) \frac{p}{p+p_{0}^{(2)}}, \\
p_{0}^{(i)}=\frac{1}{1-c}\left(\frac{1-c}{c}\right)^{c}\left(\frac{\alpha^{(2)}}{\alpha^{(1)}}\right)^{c} \frac{1}{\alpha^{(2)}} \exp \left[-\beta\left(\varepsilon_{2}^{(i)}+F_{\mathrm{int}}\right)\right], \\
\alpha^{(i)}=\frac{1}{T}\left(\frac{2 \pi \hbar^{2}}{m^{(i)} T}\right)^{3 / 2}, \quad i=1, \ldots, 2,
\end{gathered}
$$

where $a=L / N_{0}$ is the mean distance between the seats in a channel, $c$ is the concentration of the first component in the gas phase above the membrane, $c_{1}$ and $c_{2}$ are the concentrations of the components in a channel, $\sigma_{1}$ and $\sigma_{2}$ are the diameters of molecules of the first and second species, $\sigma_{\mathrm{av}} \equiv\left(\sigma_{1}+\sigma_{2}\right) / 2$ is the average diameter, $\theta$ is the fill factor of the channel, and $F_{\text {int }}$ has the meaning of the free energy of interaction per particle in the system of atomic particles in the channel. The concentrations of the components in the channel are chosen so that the following relation is satisfied:

$$
c_{1}+c_{2}=1
$$

In accordance with relations (94), the calculation of the adsorption isotherm is reduced to the calculation of the value of $F_{\text {int }}$ :

$$
F_{\text {int }}=-T \ln \left(\frac{Q_{N}}{L^{N}}\right)
$$

where

$$
\begin{array}{r}
Q_{N}=\int \cdots \int \exp \left[-\beta \sum_{i, m} U\left(x_{i}^{w}\right)\right] d x_{1}^{w} \cdots d x_{N}^{w} \\
i=1 \ldots N, w=1,2
\end{array}
$$

$U(x)$ is the potential energy of interaction of particles of species $w$ separated by distance $x$ from each other. It is convenient for subsequent computations to introduce, instead of coordinates $x_{i}$ of individual particles in a channel, "pair" coordinates $\zeta_{i}^{l m}$, where $l=1, m=1$ corresponds to the location of two particles of the first species in the vicinity of point $z_{i} ; l=2, m=2$ corresponds to the same for particles of the second species, and so on. Obviously, the coordinates are connected through the relation

$$
\sum_{1}^{N_{11}} \zeta_{i}^{11}=L_{1}, \quad \sum_{1}^{N_{12}} \zeta_{i}^{12}=L_{2}, \quad \sum_{1}^{N_{22}} \zeta_{i}^{22}=L_{2} .
$$

Here $N_{i j}$ has the meaning of the total number of pairs of closely spaced particles of species $i$ and $j$ and $L_{1}\left(L_{2}, L_{3}\right)$ is the effective length "occupied" by all pairs $N_{11}\left(N_{12}, N_{22}\right)$ of particles. Considering that $L_{1}+L_{2}+L_{3}=L$ and, as a consequence, the impossibility of mutual penetration of particles through one another, we can reduce integration with respect to coordinates $x i$ to integration with respect to coordinates $\zeta_{l m}$, which corresponds to summation over all possible configurations of pairs of particles of the first and second species in the channel. Consequently, we obtain the following expression for $Q_{N}$ from (97):

$$
\begin{aligned}
Q_{N}=\int_{0}^{\infty} \cdots \int_{0}^{\infty} & \delta\left(\sum_{1}^{N_{11}} \zeta_{i}^{11}-L_{1}\right) \delta\left(\sum_{1}^{N_{12}} \zeta_{i}^{12}-L_{2}\right) \\
& \times \delta\left(\sum_{1}^{N_{22}} \zeta_{i}^{22}-L_{3}\right) \\
& \times \delta\left(L_{1}+L_{2}+L_{3}-L\right) \exp \left[-\beta \sum U\left(\zeta_{i}^{k m}\right)\right] \\
& \times \prod_{i} d \zeta_{i}^{11} d \zeta_{i}^{12} d \zeta_{i}^{22} .
\end{aligned}
$$

Using the delta function representation in the form of a line integral [45] and carrying out integration with respect to $\zeta_{i}^{l m}$ independently for different values of indices $l$ and $m$, we obtain

$$
\begin{aligned}
Q_{N}=\frac{1}{16 \pi^{4}} \oint & \cdots \oint e^{S_{1} L_{1}}\left[\int_{0}^{\infty} \exp \left(\beta U\left(\zeta_{i}^{11}\right)-S_{1} \zeta_{i}^{11}\right)\right. \\
\left.\times \prod_{i} d \zeta_{i}^{11}\right]^{N_{11}} & \\
& \times e^{S_{2} L_{2}}\left[\int_{0}^{\infty} \exp \left(\beta U\left(\zeta_{i}^{12}\right)-S_{2} \zeta_{i}^{12}\right) \prod_{i} d \zeta_{i}^{12}\right]^{N_{12}} \\
& \times e^{S_{3} L_{3}}\left[\int_{0}^{\infty} \exp \left(\beta U\left(\zeta_{i}^{22}\right)-S_{3} \zeta_{i}^{22}\right) \prod_{i} d \zeta_{i}^{22}\right]^{N_{22}} \\
& \times e^{S\left[L-\left(L_{1}+L_{2}+L_{3}\right)\right]} d S_{1} d S_{2} d S_{3} d S .
\end{aligned}
$$

Integrating this relation with respect to $\zeta_{l m}$ for the simplest form of the intermolecular interaction potential of the hardsphere type

$$
\begin{aligned}
& U\left(\xi_{i}^{11}\right)= \begin{cases}0, & \zeta_{i}^{11}>\sigma_{1} \\
\infty, & \zeta_{i}^{11} \leqslant \sigma_{1},\end{cases} \\
& U\left(\xi_{i}^{12}\right)= \begin{cases}0, & \zeta_{i}^{12}>\sigma_{\mathrm{av}} \\
\infty, & \zeta_{i}^{12} \leqslant \sigma_{\mathrm{av}}\end{cases} \\
& U\left(\xi_{i}^{11}\right)= \begin{cases}0, & \zeta_{i}^{22}>\sigma_{2} \\
\infty, & \zeta_{i}^{22} \leqslant \sigma_{2},\end{cases}
\end{aligned}
$$


we obtain

$$
\begin{aligned}
Q_{N}= & \frac{\Gamma\left(N c_{1}^{2}+1\right) \Gamma\left(N c_{1} c_{2}+1\right) \Gamma\left(N c_{2}^{2}+1\right)}{4 \pi} \frac{1}{N} \\
& \times\left[L-N\left(\sigma_{1} c_{1}^{2}+\sigma_{2} c_{2}^{2}+2 \sigma_{\mathrm{av}} c_{1} c_{2}\right)\right]^{N} .
\end{aligned}
$$

Here we have used the definition $N_{i j}=N c_{i} c_{j}$ ( $N$ is the total number of particles in a channel) and the representation of a factorial in terms of the gamma function, $N !=\Gamma(N+1)$. Eliminating the factor corresponding to an ideal system of atomic particles from relation (102) and substituting the result into (96), we obtain the following expression for $F_{\text {int }}$ :

$$
F_{\mathrm{int}}=-T \ln (1-C \theta), \quad C \equiv \frac{1}{\sigma}\left(\sigma_{1} c_{1}^{2}+\sigma_{2} c_{2}^{2}+2 \sigma_{\mathrm{av}} c_{1} c_{2}\right) .
$$

As $c_{1} \rightarrow 0$ or $c_{2} \rightarrow 0$, expression (103) is transformed into the corresponding expression for the free energy of a one-component system of atomic particles in a $1 \mathrm{D}$ channel (Section 2.1). From relations (103) and (94) for the adsorption isotherm of a two-component system of atomic particles, we finally derive the expressions

$$
\begin{gathered}
\frac{a}{\sigma_{\mathrm{av}}} \theta=c \frac{p(1-C \theta)}{p(1-C \theta)+\tilde{p}_{0}^{(1)}}+(1-c) \frac{p(1-C \theta)}{p(1-C \theta)+\widetilde{p}_{0}^{(2)}}, \\
\widetilde{p}_{0}^{(i)}=\frac{1}{1-c}\left(\frac{1-c}{c}\right)^{c}\left(\frac{\alpha^{(2)}}{\alpha^{(1)}}\right)^{c} \frac{1}{\alpha^{(2)}} \exp \left[-\beta \varepsilon_{2}^{(i)}\right], \quad i=1, \ldots, 2 .
\end{gathered}
$$

Using these relations and specifying the external conditions, we can obtain the fill factor and the component concentration in the channel. For $c=0$ and $c=1$, isotherm (104) transforms into the isotherm for a one-component system of atomic particles, which was obtained in Section 2.1. Let us now analyze the ground state of a mixture in $1 \mathrm{D}$ channels. It is known (Section 2.1) that short-lived clusters can be formed in 1D channels in the case of a one-component system of atomic particles. The formation of clusters can apparently be expected in the case of a two-component system of atomic particles also. In order to describe possible transition to an inhomogeneous clustered state, we must calculate and analyze the free energy of the system taking into account fluctuations and the equation determining the amplitude of these fluctuations in the system (equation for the order parameter), which follows from the free energy. Since molecular transport depends on the lifetime of clusters, the analysis of the equation for the order parameter aims at determining the characteristic lifetime of clusters as a function of the channel fill factor and, hence, as a fucntion of pressure, temperature, and composition of the mixture above the membrane. In order to describe the ground state of the $1 D$ system and relaxation process to the ground state, one is to know the free energy $F$ of the $1 D$ system and two-component functional $\Delta\left[n_{\alpha}\right], \alpha=1,2$, determined by the relations (39), (44). The functional $\Delta\left[n_{\alpha}\right]$ can be written in the way of (49):

$$
\Delta\left[n_{\alpha}\right]=\Delta_{0}\left[n_{\alpha}\right]+\Delta_{\text {int }}\left[n_{\alpha}\right] .
$$

Here $\Delta_{0}\left[n_{\alpha}\right]$ is the functional of the non-interacting system and $\Delta_{\text {int }}\left[n_{\alpha}\right]$ corresponds to the particles interaction:

$$
\begin{aligned}
& \Delta_{0}\left[n_{\alpha}\right]=\lim _{\tau \rightarrow \infty} \tau^{-1} \int_{0}^{\tau} F_{0}\left[n_{\alpha}(x, t)\right] d t, \\
& \Delta_{\text {int }}\left[n_{\alpha}\right]=\lim _{\tau \rightarrow \infty} \tau^{-1} \int_{0}^{\tau} F_{1}\left[n_{\alpha}(x, t)\right] d t,
\end{aligned}
$$

where $F_{0}$ is the free energy per temperature unit of the ideal (non-interacting) system, $F_{1}$ is the fluctuation part of the free energy due to interatomic interaction in the mixture. Quantity $\Delta$ can be expressed in terms of the response function $\beta_{\alpha, \beta}\left(x, x^{\prime}, t, t^{\prime}\right)$ of the two-component system, which is defined as

$$
\delta n_{\alpha}(x, t)=\int \beta_{\alpha \beta}\left(x, x^{\prime} t, t^{\prime}\right) e V_{\beta}^{\text {ext }}\left(x^{\prime}, t^{\prime}\right) d x^{\prime} d t^{\prime},
$$

where $\delta n_{\alpha}(x, t)$ is the density fluctuation of particles of species $\alpha$ and $e V_{\text {ext }}\left(x^{\prime}, t^{\prime}\right)$ is a weak external field depending on time. Here and in the following, we assume summation over recurrent indices. Response function $\delta n_{\alpha}(x, t)$ can be derived by varying directly the mean value of functional $\Delta$ over $\delta n(x, t)[38]$ :

$$
\beta_{\alpha \beta}\left(x, x^{\prime}, t, t^{\prime}\right)=-\left(\frac{\delta^{2} \Delta}{\delta n_{\alpha}(x, t) \delta n_{\beta}(x, t)}\right)^{-1} .
$$

Using this relation, we can obtain the expansion of functional $\Delta$ into a series in deviations $\delta n$ of density from its mean value in the case of a multicomponent system. In the Fourier representation, the result has the form

$$
\begin{aligned}
\Delta= & \frac{1}{2} \int d k d \omega \beta_{\alpha \beta}^{-1}(k, \omega) \delta n_{\alpha}(k, \omega) \delta n_{\beta}^{*}(k, \omega) \\
+ & \frac{1}{3} \int d k d \omega d k^{\prime} d \omega^{\prime} \frac{\delta \beta_{\alpha \beta}^{-1}(k, \omega)}{\delta n_{\gamma}\left(k^{\prime}, \omega^{\prime}\right)} \\
& \times \delta n_{\alpha}(k, \omega) \delta n_{\beta}^{*}(k, \omega) \delta n_{\gamma}\left(k^{\prime}, \omega^{\prime}\right) \\
+ & \frac{1}{4} \int d k d \omega d k^{\prime} d \omega^{\prime} d k^{\prime \prime} d \omega^{\prime \prime} \frac{\delta^{2} \beta_{\alpha \beta}^{-1}(k, \omega)}{\delta n_{\gamma}\left(k^{\prime}, \omega^{\prime}\right) \delta n_{\delta}^{*}\left(k^{\prime \prime}, \omega^{\prime \prime}\right)} \\
& \times \delta n_{\alpha}(k, \omega) \delta n_{\beta}^{*}(k, \omega) \delta n_{\gamma}\left(k^{\prime}, \omega^{\prime}\right) \delta n_{\delta}^{*}(k, \omega)+\cdots
\end{aligned}
$$

Here $\beta_{\alpha \beta}(k, \omega)$ is the response function of the multicomponent system in the Fourier representation, and the asterisk indicates complex conjugation. It follows from the fluctuation-dissipative theorem [98] that response function $\beta_{\alpha \beta}(k, \omega)$ is directly proportional to the pair distribution; this enables us to calculate $\beta_{\alpha \beta}(k, \omega)$ for a $1 \mathrm{D}$ system with an arbitrary density, for which the pair distribution is known exactly [45]. To calculate $\Delta$, we use the local approximation that makes it possible to replace functional derivatives by ordinary derivatives [38]:

$$
\frac{\delta^{2} \varphi}{\delta \rho\left(x_{1}\right) \delta \rho\left(x_{2}\right)} \approx \delta\left(x-\mathbf{x}_{1}\right) \delta\left(x-x_{2}\right) \frac{d^{2} \varphi}{d \bar{\rho}^{2}} .
$$


Using this relation, we obtain from (109)

$$
\begin{aligned}
& \Delta=\frac{1}{2} \int d k d \omega \beta_{\alpha \beta}^{-1}(k, \omega) \delta n_{\alpha}(k, \omega) \delta n_{\beta}^{*}(k, \omega) \\
& +\frac{1}{3} \int d k d \omega d k^{\prime} d \omega^{\prime} \frac{\partial \beta_{\alpha \beta}^{-1}(k, \omega)}{\partial n_{\gamma}\left(k^{\prime}, \omega^{\prime}\right)} \\
& \quad \times \delta n_{\alpha}(k, \omega) \delta n_{\beta}^{*}(k, \omega) \delta n_{\gamma}\left(k^{\prime}, \omega^{\prime}\right) \\
& +\frac{1}{4} \int d k d \omega d k^{\prime} d \omega^{\prime} d k^{\prime \prime} d \omega^{\prime \prime} \frac{\partial^{2} \beta_{\alpha \beta}^{-1}(k, \omega)}{\partial n_{\gamma}\left(k^{\prime}, \omega^{\prime}\right) \partial n_{\delta}^{*}\left(k^{\prime \prime}, \omega^{\prime \prime}\right)} \\
& \quad \times \delta n_{\alpha}(k, \omega) \delta n_{\beta}^{*}(k, \omega) \delta n_{\gamma}\left(k^{\prime}, \omega^{\prime}\right) \delta n_{\delta}^{*}(k, \omega)+\cdots
\end{aligned}
$$

Thus, the evaluation of functional (111) in the onedimensional case can be reduced to the calculation of response functions. In the case of two-component systems, the equations for determining partial response functions $\beta$ have the form [38]

$$
\begin{gathered}
\widehat{\beta}(k, \omega)=\widehat{\beta}^{(0)}(k, \omega)+\widehat{\beta}^{(0)}(k, \omega) \widehat{R}(k, \omega) \widehat{\beta}(k, \omega), \\
\widehat{\beta}(k, \omega) \equiv \beta_{i k}(k, \omega)=\left(\begin{array}{ll}
\beta_{11}(k, \omega) & \beta_{12}(k, \omega) \\
\beta_{21}(k, \omega) & \beta_{22}(k, \omega)
\end{array}\right), \\
\widehat{R}(k, \omega) \equiv R_{i k}(k, \omega)=\left(\begin{array}{ll}
R_{11}(k, \omega) & R_{12}(k, \omega) \\
R_{21}(k, \omega) & R_{22}(k, \omega)
\end{array}\right), \\
\widehat{\beta}^{(0)}(k, \omega) \equiv \beta_{i k}^{(0)}(k, \omega)=\left(\begin{array}{ll}
\beta_{11}^{(0)}(k, \omega) & \beta_{12}^{(0)}(k, \omega) \\
\beta_{21}^{(0)}(k, \omega) & \beta_{22}^{(0)}(k, \omega)
\end{array}\right) .
\end{gathered}
$$

Here $\beta_{i k}(0)(k, \omega)$ and $\beta_{i k}(k, \omega)$ are the partial response functions for noninteracting and interacting particles, respectively. The quantity $R_{i k}(k, \omega)$ describes the effective dynamic interaction between particles and is defined as

$$
R_{i k}(k, \omega)=\frac{\delta^{2} \Delta_{\text {int }}}{\delta n_{i}(k, \omega) \delta n_{k}(k, \omega)} .
$$

Premultiplying expression (112) by $\left(\widehat{\beta}^{(0)}(k, \omega)\right)^{-1}$ and postmultiplying it by $\widehat{\beta}^{-1}(k, \omega)$, we obtain

$$
\left(\widehat{\beta}^{(0)}(k, \omega)\right)^{-1}=\widehat{\beta}^{-1}(k, \omega)+\widehat{R}(k, \omega) .
$$

Over long time periods $\left(t \gg \tau_{\text {mom }}\right)$, function $R_{i k}(k, \omega)$ can be represented in the form

$$
\widehat{R}(k, 0)=\left(\widehat{\beta}^{(0)}(k, 0)\right)^{-1}-\widehat{\beta}^{-1}(k, 0) .
$$

In view of homogeneity and the absence of correlations in a system of noninteracting particles, we have $\widehat{\beta}^{(0)}(k, 0)=$ $\widehat{\beta}^{(0)}(0,0)=b_{i j}$, where

$$
b_{i j} \equiv-\frac{\partial^{2} S}{\partial c_{i} \partial c_{j}}
$$

with $S$ being the entropy of the two-component system of atomic particles divided by temperature in the absence of the intermolecular interaction. In this case, we obtain the following expression for the effective interaction from (115):

$$
\widehat{R}(k, 0)=\left(\widehat{\beta}^{(0)}(0,0)\right)^{-1}-\widehat{\beta}^{-1}(k, 0) .
$$

Substituting this expression into (114), we obtain the following relation for response function $\widehat{\beta}(k, \omega)$ :

$$
\widehat{\beta}^{-1}(k, \omega)=\left(\widehat{\beta}^{(0)}(k, \omega)\right)^{-1}+\widehat{\beta}^{-1}(k, 0)-\left(\widehat{\beta}^{(0)}(0,0)\right)^{-1} .
$$

In order to calculate the response function $\widehat{\beta}(k, 0)$ for a system of interacting particles, we can use the fluctuationdissipative theorem connecting response function $\widehat{\beta}(k, 0)$ with the pair correlation function for a system of interacting particles:

$$
\beta_{i j}(k, 0)=-\left[b_{i j}^{-1}+c_{i} c_{j} \theta^{2} v_{i j}(k)\right]
$$

Here $v_{i j}(k)$ is the pair correlation function divided by temperature [46]. Relation (119) is a generalization of the relation (68) for one-component systems to two-component systems.

Thus, the calculation of the response function can be reduced to the calculation of the pair correlation function or function $g_{i k}$ called a pair distribution [46] and connected with the pair correlation function through the relation

$$
v_{i j}(k)=g_{i j}(k)-c_{i} c_{k} \delta(k) .
$$

We will calculate the pair distribution using the method described in [45] and determine $g_{11}(k)$. Functions $g_{12}(k)$ and $g_{22}(k)$ are calculated similarly. Pair distribution $g_{11}(x)$ can be written in the form [45]

$$
g_{11}(k)=\sum_{m=1}^{\infty} \Psi_{m}^{11}(k)-1
$$

where the function $\Psi_{m}^{11}(k)$ is the Fourier transform of the function $\Psi_{m}^{11}(\zeta)$, which is equal to the probability of finding two particles of the first species separated from each other by $m$ other particles and by distance $\zeta$. Function $\Psi_{m}^{11}(k)$ can be written in the form

$$
\begin{aligned}
\Psi_{m}^{11}(k)=\int d \zeta e^{i k \zeta}\left(\left(\sum_{n=0}^{N_{12} N_{22}} \sum_{m-0} \bar{Q}_{m-l, n, l}(\zeta)\right.\right. \\
\left.\cdot \bar{Q}_{N_{11}-(m-n-l), N_{12}-n, N_{22}-l}(L-\zeta)\right) \\
\left.\times\left(\bar{Q}_{N_{11}, N_{12}, N_{22}}(L)\right)^{-1}\right),
\end{aligned}
$$


where $\bar{Q}_{m, n, l}(\zeta)$ is the configuration integral corresponding to the presence of $m$ pairs of particles of the first species, $n$ pairs of particles of the first and second species, and $l$ pairs of particles of the second species in a channel. For $m=N_{11}$, $n=N_{12}$, and $l=N_{22}$, the expression for configuration integral $\bar{Q}_{m, n, l}(\zeta)$ coincides with (98) [45]:

$$
\begin{gathered}
\bar{Q}_{m, n, l}(\zeta)=\oint d S\left[\varphi_{11}(S)\right]^{m}\left[\phi_{12}(S)\right]^{n}\left[\phi_{22}(S)\right]^{l} e^{S \zeta}, \\
\phi_{i j}(S)=\int_{0}^{\infty} e^{-S x} e^{-U_{i j}(x) / T} d x .
\end{gathered}
$$

Here $\zeta$ has the meaning of a coordinate and $\phi_{i j}(S)$ is the Laplace mapping of function $f_{i j}(x) \equiv \exp \left(-U_{i j}(x) / T\right)$. In the case of an intermolecular interaction potential in the form of that in the hard-sphere model, we have

$$
\begin{aligned}
& U_{11}(x)= \begin{cases}0, & x>\sigma_{1} \\
\infty, & x \leqslant \sigma_{1},\end{cases} \\
& U_{12}(x)= \begin{cases}0, & x>\sigma_{\mathrm{av}} \\
\infty, & x \leqslant \sigma_{\mathrm{av}}\end{cases} \\
& U_{22}(x)= \begin{cases}0, & x>\sigma_{2} \\
\infty, & x \leqslant \sigma_{2} .\end{cases}
\end{aligned}
$$

Substituting these relations into (123), we obtain the following expression for the configuration integral:

$$
\begin{aligned}
\bar{Q}_{m, n, l}(\xi) & =e^{p \xi / T}\left[\phi_{11}\left(\frac{p}{T}\right)\right]^{m}\left[\phi_{12}\left(\frac{p}{T}\right)\right]^{n}\left[\phi_{22}\left(\frac{p}{T}\right)\right]^{l}, \\
\phi_{11}\left(\frac{p}{T}\right) & =\frac{T}{p} e^{-p \sigma_{1} / T}, \quad \phi_{12}\left(\frac{p}{T}\right)=\frac{T}{p} e^{-p \sigma_{\mathrm{av}} / T} \\
\phi_{22}\left(\frac{p}{T}\right) & =\frac{T}{p} e^{-p \sigma_{2} / T} .
\end{aligned}
$$

Here $p$ has the meaning of one-dimensional "pressure." Substituting relation (125) into (122), we obtain the following expression for pair distribution $g_{11}(k)$ :

$$
\begin{aligned}
g_{11}(k)= & \frac{(p / T) e^{i k \sigma_{1}}}{(p / T)-i k-(p / T) e^{i k \sigma_{1}}} \cdot \frac{1}{1-e^{i k\left(\sigma_{\mathrm{av}}-\sigma_{1}\right)}} \\
& \cdot \frac{1}{1-e^{i k\left(\sigma_{2}-\sigma_{1}\right)}} .
\end{aligned}
$$

In the long-wave approximation $\left(0 \leq k \leq 2 \pi / \max \left\{\sigma_{1}, \sigma_{2}\right\}\right)$, expression (126) contains no poles and we obtain

$$
\begin{gathered}
g_{11}(x)=\frac{1}{\theta_{\text {eff }}} \sum_{m, x-m a>0} \frac{\left(y-m\left(\sigma_{1} / a\right)\right)^{m-1}}{(m-1) !\left(1 / \theta_{\text {eff }}-1\right)^{m}} \\
\cdot \exp \left(-\frac{y-m}{1 / \theta_{\text {eff }}-1}\right), \\
y=\frac{x}{a\left(c_{1}, c_{2}\right)}, \quad \theta_{\text {eff }}=\frac{N \cdot a\left(c_{1}, c_{2}\right)}{L}, \\
a_{\mathrm{eff}}\left(c_{1}, c_{2}\right)=\sigma_{1} c_{1}^{2}+\sigma_{2} c_{2}^{2}+2 \sigma_{\mathrm{av}} c_{1} c_{2} .
\end{gathered}
$$

Functions $g_{12}(x)$ and $g_{22}(x)$ can be calculated similarly. Substituting expressions (127) and (119) into (118), we obtain the final expression for response function $\widehat{\beta}(k, 0)$ :

$$
\begin{aligned}
& (\hat{\beta}(k, 0))^{-1} \\
& \quad=\left(\begin{array}{cc}
-\frac{a_{11}}{1+a_{11} \theta^{2} c_{1}^{2} \Delta \nu_{11}(k)} & -\frac{a_{12}}{1+a_{12} \theta^{2} c_{1} c_{2} \Delta \nu_{12}(k)} \\
-\frac{a_{21}}{1+a_{21} \theta^{2} c_{1} c_{2} \Delta \nu_{21}(k)} & -\frac{a_{22}}{1+a_{22} \theta^{2} c_{2}^{2} \Delta \nu_{22}(k)}
\end{array}\right) .
\end{aligned}
$$

Here

$$
\Delta v_{i j}(k) \equiv v_{i j}(k)-v_{i j}(0), \quad a_{i j} \equiv-\frac{\partial^{2} F}{\partial c_{i} \partial c_{j}} .
$$

$F$ is the free energy of a two-component system of atomic particles with interaction, referred to temperature, which can be calculated by differentiating configuration integral $\bar{Q}_{N}$ twice with respect to concentration. Function $\widehat{\beta}(k, \omega)$ can be obtained by generalizing the response function of the onecomponent system of atomic particles,

$$
\beta_{0}^{-1}(k, \omega)=-\frac{1}{\theta}\left(1+\frac{\omega}{\omega_{0}}\right)=\frac{\partial^{2} F}{\partial \theta^{2}}\left(1+\frac{\omega}{\omega_{0}}\right)
$$

derived in Section 2.1, to the case of a two-component mixture:

$$
\begin{aligned}
& \left(\widehat{\beta}^{(0)}(k, \omega)\right)^{-1}=\left(\begin{array}{ll}
\left(1+\frac{\omega}{\omega_{1}}\right) b_{11} & \left(1+\frac{\omega}{\omega_{1}}\right) b_{12} \\
\left(1+\frac{\omega}{\omega_{2}}\right) b_{21} & \left(1+\frac{\omega}{\omega_{2}}\right) b_{22}
\end{array}\right), \\
& \omega_{1}^{(p)}=-i D_{1} k^{2}, \quad \omega_{2}^{(p)}=-i D_{2} k^{2} \text {. }
\end{aligned}
$$

Here $D_{1}, D_{2}, \omega_{1}^{(p)}$, and $\omega_{2}^{(p)}$ are the diffusion coefficients and the relaxation frequency spectra for pure components. Substituting relations (128) and (131) into (118), we obtain the following expression for response function $\beta(k, \omega)$ : 


$$
(\beta(k, \omega))^{-1}=\left(\begin{array}{ll}
\frac{\omega}{\omega_{1}^{(p)}} b_{11}-\frac{a_{11}}{1+a_{11} \theta^{2} c_{1}^{2} \Delta \nu_{11}(k)} & \frac{\omega}{\omega_{1}^{(p)}} b_{12}-\frac{a_{12}}{1+a_{12} \theta^{2} c_{1} c_{2} \Delta \nu_{12}(k)} \\
\frac{\omega}{\omega_{2}^{(p)}} b_{21}-\frac{a_{21}}{1+a_{21} \theta^{2} c_{1} c_{2} \Delta \nu_{21}(k)} & \frac{\omega}{\omega_{12}^{(p)}} b_{22}-\frac{a_{22}}{1+a_{22} \theta^{2} c_{2}^{2} \Delta v_{22}(k)}
\end{array}\right) .
$$

It is convenient for subsequent analysis to pass to new variables. For a two-component system, the relation $c_{1}+c_{2}=1$ holds and quantity $\delta n$ can be represented in the form

$$
\delta \mathbf{n}(k, \omega)=\left(\begin{array}{c}
1 \\
-1
\end{array}\right) \xi(k, \omega)
$$

where $\xi$ can be interpreted as an order parameter. Substituting (133) into (111), we obtain the expression for functional $\Delta$ :

$$
\begin{aligned}
\Delta= & \frac{1}{2} \int d k d \omega \Omega\left|\xi_{k, \omega}\right|^{2}+\frac{2}{3} \int d k d \omega \frac{\partial \Omega}{\partial n}\left|\xi_{k, \omega}\right|^{2} \xi_{k, \omega} \\
& +\int d k d \omega \frac{\partial^{2} \Omega}{\partial n^{2}}\left|\xi_{k, \omega}\right|^{4}+\cdots
\end{aligned}
$$

where

$$
\begin{aligned}
\Omega \equiv & \left(\beta_{11}(k, \omega)\right)^{-1}-\left(\beta_{12}(k, \omega)\right)^{-1} \\
& -\left(\beta_{21}(k, \omega)\right)^{-1}+\left(\beta_{22}(k, \omega)\right)^{-1} .
\end{aligned}
$$

Series (134) can be summed and written in the form

$$
\Delta=\frac{1}{2} \int d k d \omega|\xi|_{k, \omega}^{2} \Omega\left(n, c+\xi_{k, \omega}, k, \omega\right) .
$$

The formal proof of the correctness of representing quantity $\Delta$ in form (136) without analyzing the convergence of the corresponding series follows from the expansion of expression (136) into a power series of order parameter $\xi$. This expansion exactly reproduces series (134) (at least, to within terms on the order of $\xi^{5}$ ). Using relations (132), (135), and (136), we can obtain the dependence of functional $\Delta$ on the wave vector and order parameter of the system. The minimum of functional $\Delta$ determines the ground state of the system. Substituting relation (132) into (136), evaluating the derivative of $\Delta$ with respect to the order parameter, and applying the inverse Fourier transformation in frequency $\omega$ to the resulting relation, we obtain

$$
\frac{\partial \xi_{k}}{\partial t}=-\widetilde{D} k^{2} \frac{\partial\left(F_{1}\left(\xi_{k}, k\right)\right)}{\partial \xi_{k}}
$$

where

$$
\begin{gathered}
\widetilde{D}=\left(\frac{b_{11}-b_{12}}{D_{1}}+\frac{b_{22}-b_{21}}{D_{2}}\right)^{-1}, \\
F_{1}\left(\xi_{k}, k\right) \equiv \frac{1}{2} \Omega\left(c+\xi_{k}, k\right) \xi_{k}^{2}, \\
\Omega(c, k)=-\frac{a_{11}}{1+a_{11} \theta^{2} c_{1}^{2} \Delta \nu_{11}(k)}+\frac{2 a_{12}}{1+a_{12} \theta^{2} c_{1} c_{2} \Delta \nu_{12}(k)} \\
-\frac{a_{22}}{1+a_{22} \theta^{2} c_{2}^{2} \Delta v_{22}(k)} .
\end{gathered}
$$

Equation (137) has the same form as the equation usually used for the order parameter. The role of the order parameter is played in this case by the quantity defined by relation (133). It should be noted that the order parameter here could be either positive or negative. The cases when $\xi>$ 0 and $\xi<0$ correspond to the density fluctuation of the first and the second components, respectively. The role of the diffusion coefficient is played by quantity $\widetilde{D}$ depending on the fill factor and the concentration of components in channels via coefficients $a_{i j}$ and $b_{i j}$ defined by relations (116) and (128). It should be noted that the concentration of the components in a channel (and its fill factor) could be determined unambiguously from isotherm (104) proceeding from the pressure, temperature, and composition of the system of atomic particles. Analysis of functional $F_{1}$ as a function of order parameter $\xi$ and wave vector $k$ makes it possible to investigate the possibility for a transition of the system to an inhomogeneous state. For example, when the minimum of functional $F_{1}$ is attained for $\xi \neq 0, k=0$, a conventional phase transition to a homogeneous state takes place [99]. The minimum at $\xi=0, k \equiv k_{c} \neq 0$ corresponds to the propagation of a density wave over a distance of $r \sim k_{c}^{-1}$. In the case when the minimum is attained at $\xi \neq 0$ and $k \neq 0$, a transition to an inhomogeneous state with clusters formed in the system is realized [99]. Local minima here determine metastable states. Figure 13 shows the graphs illustrating the dependence of $F_{1}$ on order parameter $\xi_{k}$ and wave vector $k$ for a mixture in which one of the gases (first) is a strong sorbate, while the other gas is a weak sorbate. The chosen parameters $\left(a=3.8 \AA, \sigma_{1}=4.3 \AA, \sigma_{2}=3.6 \AA, \varepsilon_{1}=0.61 \mathrm{eV}\right.$, $\varepsilon_{2}=0.38 \mathrm{eV}, p=2000 \mathrm{kPa}$, and $\left.T=300 \mathrm{~K}\right)$ correspond to a methane-butane molecular mixture [51] and make it possible to describe adsorption and fluxes of these one-component gases in zeolite membranes. It should be noted that the choice of parameters $p, T$, and $c$ determines unambiguously the filling factor $\theta$ and concentrations $c_{1}$ and $c_{2}$ of gases in a channel. 


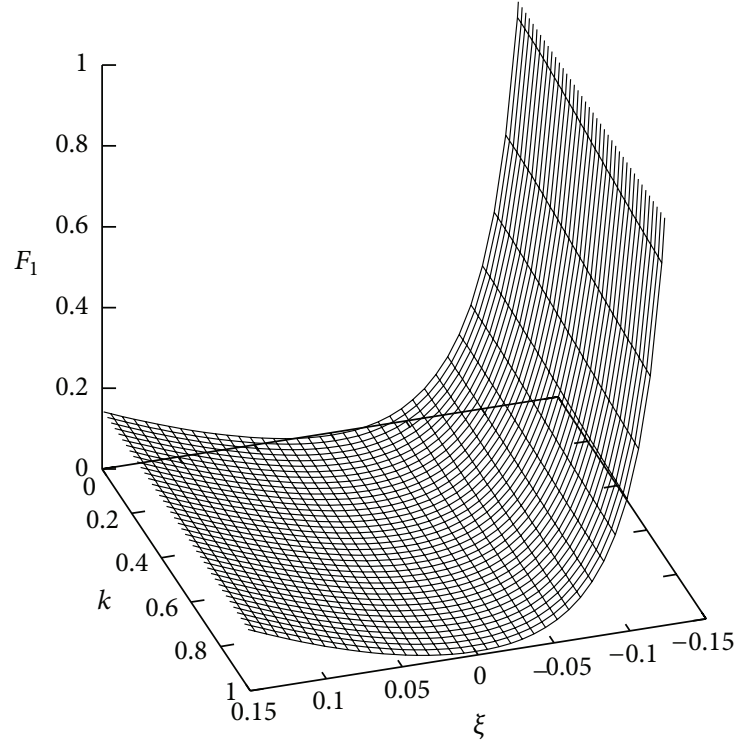

(a)

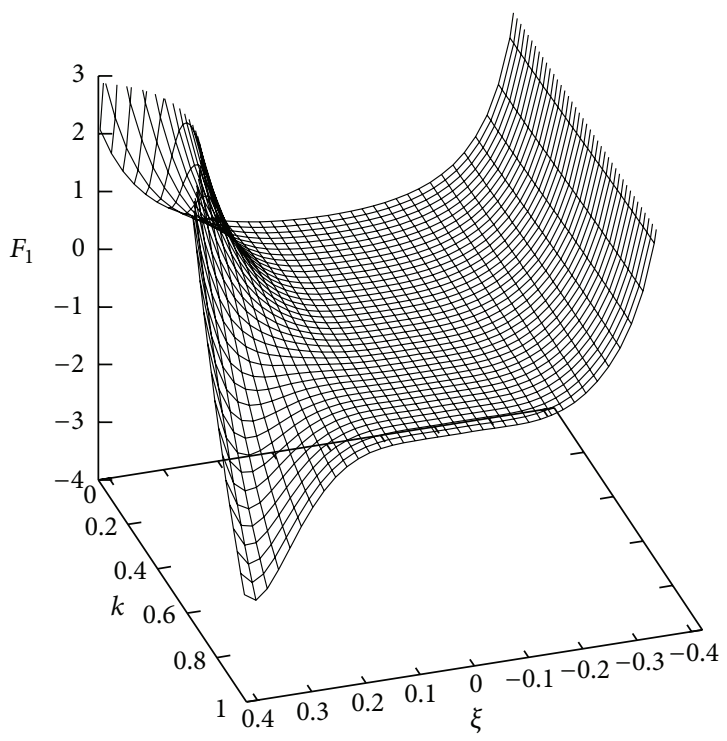

(c)

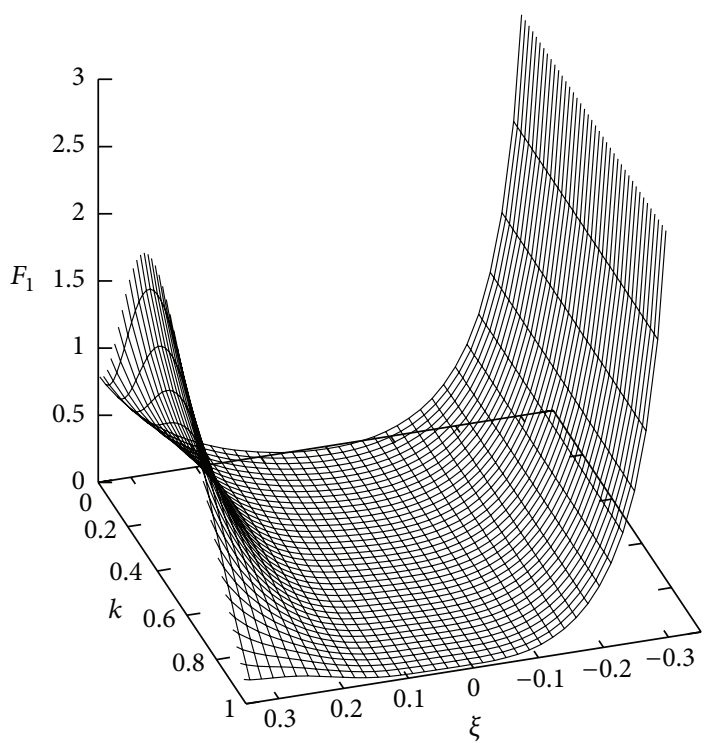

(b)

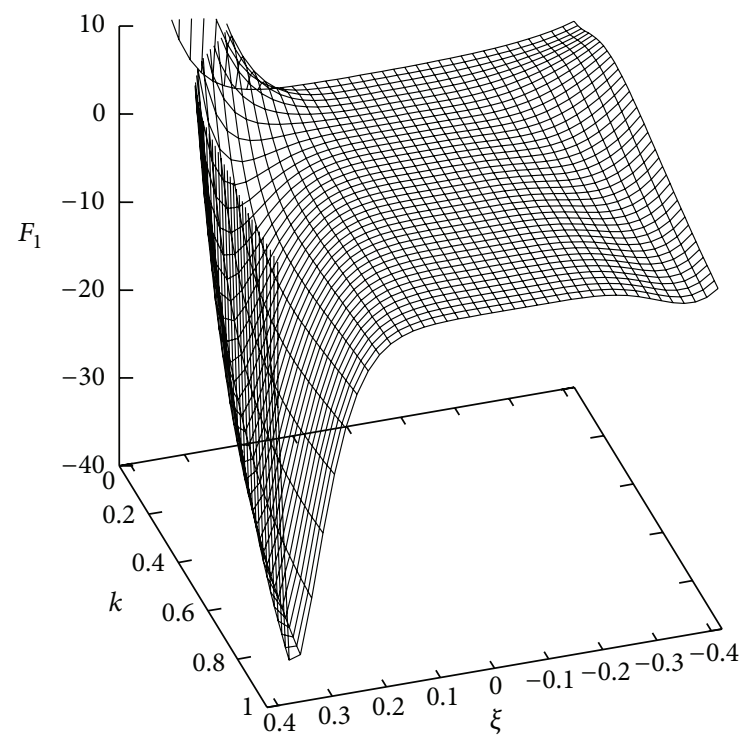

(d)

Figure 13: Dependence of free energy $F_{1}$ on the order parameter and wave vector for various concentrations of the first component in the mixture: (a) $c=0.1(\theta=0.67),(b) c=0.25(\theta=0.71),(c) c=0.3(\theta=0.74)$, and (d) $c=0.45(\theta=0.75)$.

For low concentrations of the first component in a gas mixture and for fill factors $\theta \leq 0.7$, functional $F_{1}$ has only one minimum at $\xi=0, k=0$ (Figures 13(a) and 13(b)). This corresponds to a homogeneous state of the system. An increase in the concentration of the first component leads to the emergence of local minima in functional $F_{1}$.

In this case, the global minimum is shifted to point $\xi=$ $\xi_{c}>0, k=k_{c}$. In this case, the ground state of the system becomes spatially inhomogeneous, which corresponds to the formation of clusters in the system. This situation will be analyzed in greater detail later. Equation (137) for the order parameter also allows us to calculate the characteristic relaxation times for the emerging density fluctuation depending on external conditions. For this purpose, we expand functional $F_{1}\left(\xi_{k}, k\right)$ into a series in the vicinity of $\xi_{k}=0$ to within the first nonvanishing term:

$$
F_{1}\left(\xi_{k}, k\right) \sim \lambda(k) \xi_{k}^{2} .
$$

Substituting (139) into (137), we obtain

$$
\frac{\partial \xi_{k}}{\partial t}=-\frac{\xi_{k}}{\tau}, \quad \tau=\left(2 \lambda(k) \widetilde{D} k^{2}\right)^{-1} .
$$

The quantity $\tau$ is the lifetime of the $k$ th mode of the density fluctuation emerging in the vicinity of $\xi_{k}=0$. The curves describing the lifetime of the emerging fluctuation as a function of the wave vector for different mixture compositions are shown in Figure 14. It can be seen from Figure 14 that, 


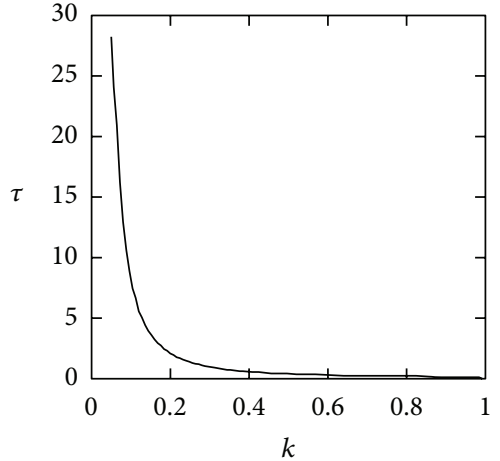

(a)

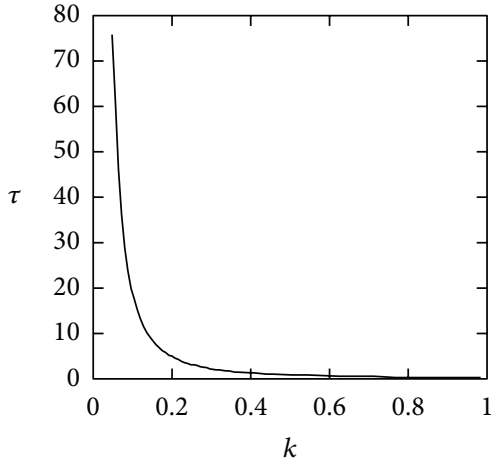

(b)

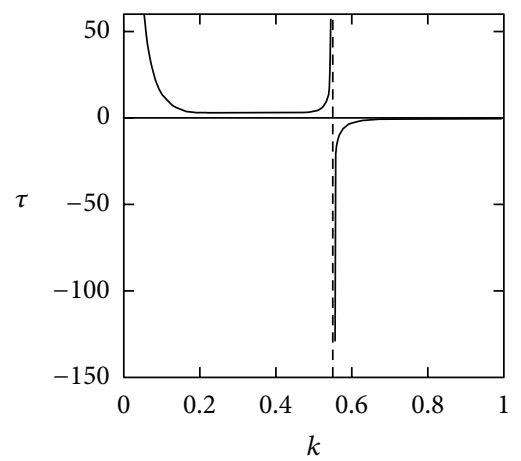

(c)

FIGURE 14: Dependence of lifetime $\tau$ on wave vector $k$ for $c=0.1$ (a), 0.3 (b), and 0.45 (c).

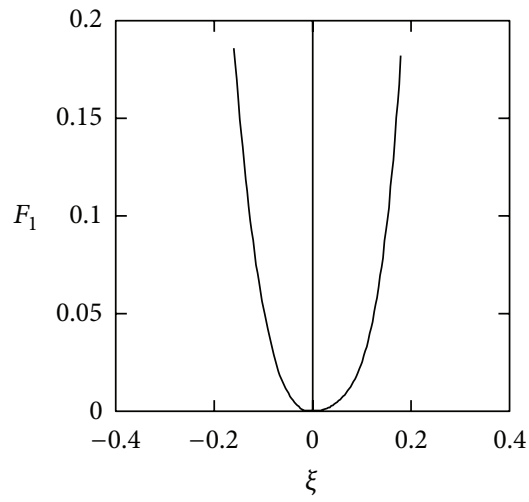

(a)

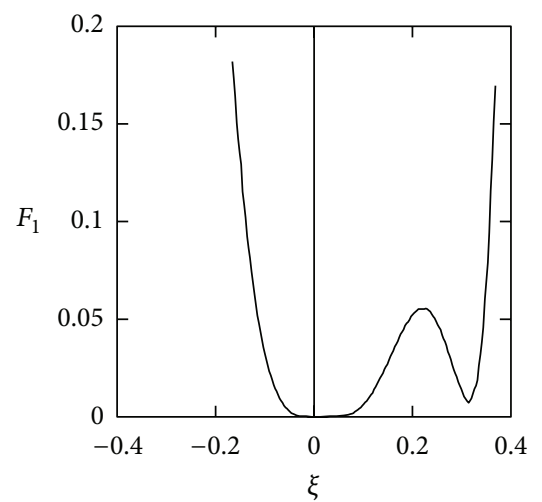

(b)

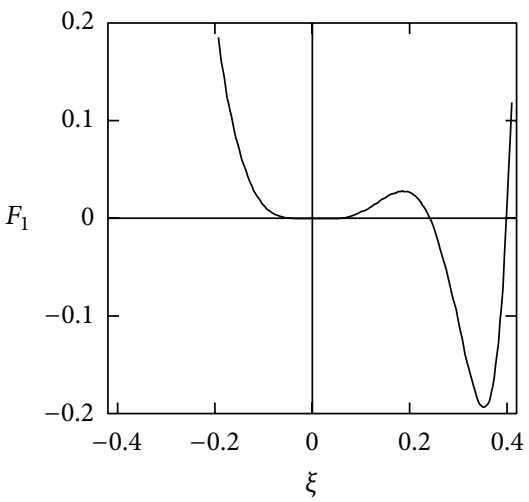

(c)

Figure 15: Dependence of free energy $F_{1}$ on the order parameter for different values of the wave vector for $c=0.3: k=0.1$ (a), 0.3 (b), and 0.5 (c).

for a low concentration of the highly adsorbed component, the lifetime decreases monotonically with increasing wave vector, while the free energy has a minimum at $\xi=0$. From the physics standpoint, this means that the state of the system in a channel for a given composition of the mixture is homogeneous and the growth of clusters is energetically disadvantageous. It should be noted that the fill factor of the channel is small in this case, but short-lived clusters with the lifetime

$$
\tau \sim \frac{r^{2}}{D}=\frac{r^{2}}{D_{0}} \exp \left(\frac{E_{a}}{T}\right)
$$

can be formed due to density fluctuations. Here $D=$ $D_{0} \exp \left(-E_{a} / T\right)$ is the diffusion coefficient of solitary particles, $E_{a}$ is the diffusion activation energy, and $r$ is the characteristic size of fluctuations. Characteristic size $r$ is determined, as in Section 2.1, from the position of the extremum of the imaginary part of the spectrum divided by the square of the wave vector. It should be noted that the value of $r$ increases with the fill factor of the channel Section 2.1. Expression (142) can be derived by expanding order parameter (137) into a series in the vicinity of $\xi=0$.
For low concentrations, expression (141) coincides with the characteristic diffusion decay time for density fluctuations.

An increase in the concentration of the first component of the mixture to $c=0.3$ elevates the fill factor of the channel to $\theta=0.74$ (see Figure 13(c)), while $F_{1}$ acquires two more minima for $k \sim 1$. Figure 15 shows the dependences of the free energy on the order parameter for different values of the wave vector at $c=0.3$. It can be seen from the figure that the free energy minimum is attained at point $\xi=0$ for values of the wave vector $0<k<0.4$. For $k \approx 0.5$, functional $F$ has two minima (at $\xi_{c}^{(0)}=0$ and $\xi_{c}^{(1)}=0.35$ ), and the state with $\xi_{c}^{(1)}$ is separated from the state with $\xi_{c}^{(0)}$ by a potential barrier. Since the global minimum is attained at $\xi=\xi_{c}^{(1)}$, the state with $\xi_{c}^{(0)}$ is metastable. The ground state of the system is attained at $\xi=\xi_{c}^{(1)}$ and is clustered. It should be noted that, in accordance with formula (133), clusters of the first component are formed in the channel since $\xi_{c}^{(1)}>0$.

The lifetime of the clusters can be determined by passing from (137) for the order parameter to a stochastic differential equation (Langevin equation) and the corresponding Fokker-Plank equation via the introduction of additive noise. Steady-state solutions to the Fokker-Planck equation define 


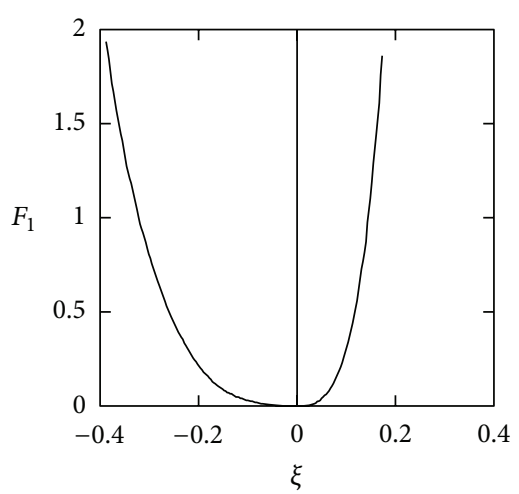

(a)

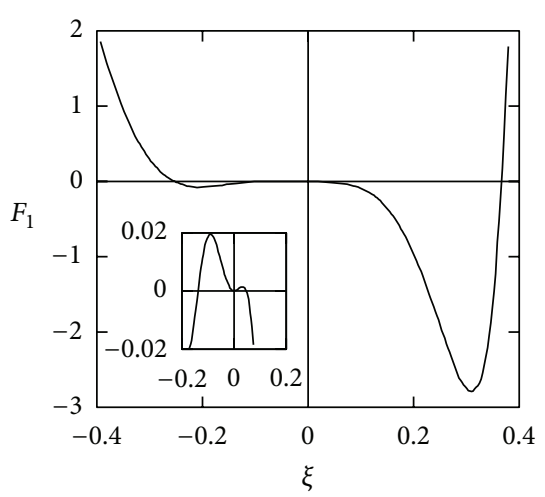

(b)

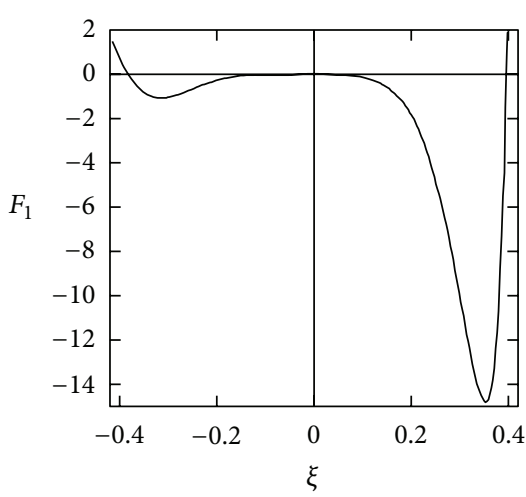

(c)

FIGURE 16: Dependence of free energy $F_{1}$ on the order parameter for different values of the wave vector for $c=0.45: k=0.1$ (a), 0.3 (b), and 0.5 (c).

the probability for the system being in the state described by order parameter $\xi_{c}^{(1)}$. For the lifetime, we have

$$
\begin{gathered}
\tau \sim \frac{r^{2}}{D_{0}} \exp \left(\frac{E_{a}}{T}-\delta F\right), \\
\delta F=F_{1}\left(c+\xi_{c}^{(1)}, k_{c}\right) \cdot T .
\end{gathered}
$$

The decay of a cluster formed in the system occurs via overcoming an energy barrier of height $\delta F$. From the standpoint of physics, this can be interpreted as an effective increase in the diffusion activation energy for the particles in the cluster. However, for $c=0.3$, the barrier height is small $(\delta F \sim 0.2 T$; see Figure 13(c)), and lifetime (142) is comparable to time (141). A further increase in the first component concentration in the mixture leads to negative values of the lifetime for a certain value of $k=k_{c}<1$ (see Figure 14(c)). In this case, clusters with a size of $r \sim k_{c}^{-1}$ are formed in the channel; for $k<k_{c}$, fluctuations decay over a finite time, while for $k>k_{c}$, the emerging density fluctuations evolve. Such a situation is typical of transitions to an inhomogeneous condensate state [99].

The curves describing the dependence of the free energy on the order parameter for different values of the wave vector are shown in Figure 16. Since functional $F_{1}$ has a single minimum at $\xi=0$ for $k \sim 0$, the state of particles in the channel is homogeneous. However, as the wave vector increases, curve $F_{1}(\xi)$ acquires two more minima separated from the state with $\xi=0$ by potential barriers. A further increase in the wave vector leads to disappearance of the barriers between the state with $\xi=0$ and the states corresponding to two other minima of $F_{1}$, and the state with $\xi=0$ becomes unstable.

Thus, for a low concentration of the highly adsorbed component, the fill factor of the channel is small and the state of the system in the channel is homogeneous. As the concentration of the highly adsorbed component increases, the fill factor of the channel increases, and short-lived clusters of the highly adsorbed component can be formed in the channel. A further increase in the concentration (and the fill factor of the channel) leads to an increase in the lifetime of the clusters formed in the channel. At a certain concentration of the highly adsorbed component, the clusters in the channel become long lived and the ground state of the system becomes clustered.

The formation of clusters of one of the components in a channel may strongly affect the transport of the gas through the membrane (see Section 2.1). In the next chapter, partial gas fluxes through the membrane will be calculated, and the effect of clusters on the mechanisms of transport will be analyzed.

\subsubsection{Transport in a Two-Component Gas in a High-Density} $1 D$ System. In order to calculate partial fluxes and to analyze the transport, we will use the approach proposed in Section 2.1. It will be shown in the following that the evaluation of the flux can be reduced to the calculation of the relaxation frequency spectra $\omega(\mathbf{k})$ for density fluctuations of the components. We can judge the mechanism of particle transport in a subnanometer channel from the type of the dependence of the spectrum on the wave vector of the system. We can write the expression determining the relaxation of the Fourier component $n(k, t)$ of the number density of particles in a channel in the case of an arbitrary density $n(k, t)$ under the conditions of slight deviation from equilibrium (see Section 2.1 and [100]):

$$
\dot{n}(k, t)=i \omega(k) n(k, t) .
$$

It follows from this relation that the equation for fluctuation amplitude $\delta n$ can be written in the form

$$
\delta \dot{n}(k, t)=i \omega(k) \delta n(k, t),
$$

where $\omega(k)$ is the relaxation frequency spectrum for the system under study; in the case of diffusion of noninteracting particles, this spectrum has the form [98]

$$
\omega=\omega_{0}(k)=-i D k^{2} .
$$

Equation (144) describes relaxation of the $k$ th component of density fluctuation for an arbitrary value of the wave vector. In 
particular, this equation for $k \neq 0$ makes it possible to describe the relaxation of density fluctuations and the propagation of a perturbation over a finite-size cluster in the case of its formation. For $k \rightarrow 0$, (144) describes the relaxation of density fluctuation on a large spatial scale. This quantity is associated with macroscopic fluxes. In order to calculate the fluxes, we write the continuity equation

$$
\dot{n}+\frac{\partial j}{\partial x}=0
$$

and apply the Fourier transformation to this equation:

$$
i k j(k, t)=-\dot{n}(k, t) \text {. }
$$

Substituting (143) into (147), we obtain the following relation for flux $j(k)$ :

$$
j(k, t)=\frac{n(k, t) \omega(k)}{k} .
$$

For partial fluxes, we have

$$
j_{i}(k, t)=\frac{n_{i}(k, t)}{k} \omega_{i}(k),
$$

where $n_{i}$ is the density of the $i$ th component and $\omega_{i}$ is the corresponding spectrum. The total gas flux is defined as the sum of partial fluxes of the components:

$$
\sum_{i=1}^{2} j_{i}(k, t)=j(k, t)
$$

It follows from (149) that partial fluxes are determined by relaxation frequency spectra $\omega_{i}(k)$. Thus, the problem is reduced to computing these spectra. The relaxation frequency spectra can be determined from the condition of the existence of nonzero density fluctuations for each component in an arbitrarily weak external field. Consequently, applying the Fourier transformation to (107), we find that the relaxation frequency spectrum can be determined by solving the following system of homogeneous equations:

$$
\beta^{-1}(k, \omega)\left(\begin{array}{c}
1 \\
-1
\end{array}\right)=0
$$

Here matrix $\beta^{-1}(k, \omega)$ of the response functions is defined by relation (132). In view of $\delta$-function singularities for $k \rightarrow 0$ that emerge in pair correlation functions (120) appearing in (132), we must calculate spectra $\omega_{i}(k)$ and the values of $\omega_{i}(k=$ $0)$, after which the renormalization procedure must be carried out. Using (132) and passing to the limit $k \rightarrow 0$ for spectra $\omega_{i}(k=0)$,

$$
\beta^{-1}(0, \omega)\left(\begin{array}{c}
1 \\
-1
\end{array}\right)=\left(\begin{array}{c}
\frac{\omega}{\omega_{1}^{(p)}} b_{11}-\frac{\omega}{\omega_{1}^{(p)}} b_{12}+a_{11}-a_{12} \\
\frac{\omega}{\omega_{2}^{(p)}} b_{22}-\frac{\omega}{\omega_{2}^{(p)}} b_{21}+a_{22}-a_{21}
\end{array}\right)=0,
$$

where $a_{i j}, b_{i j}, \omega_{1}^{(0)}$, and $\omega_{2}^{(p)}$ are defined by relations (116), (129), and (131). For $\omega^{(0)} \equiv \omega_{i}(k=0)$, we obtain from relation (152)

$$
\begin{aligned}
& \omega_{1}^{(0)}=-\frac{\omega_{1}^{(p)}\left(a_{11}-a_{12}\right)}{b_{11}-b_{12}}, \\
& \omega_{2}^{(0)}=-\frac{\omega_{2}^{(p)}\left(a_{22}-a_{21}\right)}{b_{22}-b_{21}} .
\end{aligned}
$$

On the other hand, substituting (132) into (152), we obtain the following expressions for relaxation frequency spectra $\omega_{i}(k)$ of the system under investigation:

$$
\begin{aligned}
& \widetilde{\omega}_{1}(k)=\frac{i D_{1} k^{2}}{b_{11}-b_{12}}\left[\frac{1}{\theta^{2} c_{1}^{2} v_{11}(k)+b_{11}^{-1}}-\frac{1}{\theta^{2} c_{1} c_{2} \nu_{12}(k)+b_{12}^{-1}}\right], \\
& \widetilde{\omega}_{2}(k)=\frac{i D_{2} k^{2}}{b_{22}-b_{21}}\left[\frac{1}{\theta^{2} c_{2}^{2} \nu_{22}(k)+b_{22}^{-1}}-\frac{1}{\theta^{2} c_{1} c_{2} \nu_{21}(k)+b_{21}^{-1}}\right] .
\end{aligned}
$$

Tildes indicate that $\widetilde{\omega}_{i}(k=0) \neq \omega_{i}^{(0)}\left(\omega_{i}^{(0)}\right.$ are defined in relations (153)).

We will carry out the renormalization procedure for spectra (154) taking into account relations (153), that is, imposing the requirement

$$
\widetilde{\omega}_{i}=\omega_{i}^{(0)}(k=0) .
$$

Taking into account the value of $\omega_{i}^{(0)}$, we find from relations (153) and (154) that

$$
a_{11}-a_{12}=\frac{b_{11}}{b_{11} \theta^{2} c_{1}^{2} v_{11}(k)+1}-\frac{b_{12}}{b_{12} \theta^{2} c_{1} c_{2} \nu_{12}(k)+1}
$$

or

$$
a_{11}=\frac{b_{11}}{b_{11} \theta^{2} c_{1}^{2} v_{11}(k)+1}, \quad a_{12}=\frac{b_{12}}{b_{12} \theta^{2} c_{1} c_{2} v_{12}(k)+1} .
$$

Similarly, for $a_{22}$, we have

$$
a_{22}=\frac{b_{22}}{b_{22} \theta^{2} c_{2}^{2} v_{22}(k)+1} .
$$

In order to separate singularities of pair correlation function $v_{i j}(k)$ for $k \rightarrow 0$, we expand it into a series in the vicinity of $k=0$ to within first-order terms. Using relations (154), we then obtain

$$
\begin{gathered}
\widetilde{\omega}_{1}(k)=\frac{i D_{1} k^{2}}{b_{11}-b_{12}}\left[\frac{1}{\theta^{2} c_{1}^{2}\left(\nu_{11}(0)+k v_{11}^{\prime}(k)\right)+b_{11}^{-1}}\right. \\
\left.-\frac{1}{\theta^{2} c_{1} c_{2}\left(\nu_{12}(0)+k v_{12}^{\prime}(k)\right)+b_{12}^{-1}}\right], \\
=\frac{i D_{1} k^{2}}{b_{11}-b_{12}}\left[\frac{a_{11}}{1+k a_{11} \theta^{2} c_{1}^{2} v_{11}^{\prime}(k)}\right. \\
\left.-\frac{a_{12}}{1+k a_{12} \theta^{2} c_{1} c_{2} v_{12}^{\prime}(k)}\right] .
\end{gathered}
$$


Taking into account the long-wave approximation and the smallness of $k$, replacing the derivative in the last expression by the difference $k v_{i k}^{\prime}(k) \approx v_{i j}(k)-v_{i j}(0)$, omitting primes, and carrying out similar calculations for $\omega_{2}$, we finally obtain

$$
\begin{aligned}
& \omega_{1}(k)=\frac{i D_{1} k^{2}}{b_{11}-b_{12}}\left[\frac{a_{11}}{1+a_{11} \theta^{2} c_{1}^{2}\left(\nu_{11}(k)-v_{11}(0)\right)}\right. \\
& \left.-\frac{a_{12}}{1+a_{12} \theta^{2} c_{1} c_{2}\left(\nu_{12}(k)-v_{12}(0)\right)}\right] \text {, } \\
& \omega_{2}(k)=\frac{i D_{2} k^{2}}{b_{22}-b_{21}}\left[\frac{a_{22}}{1+a_{22} \theta^{2} c_{2}^{2}\left(\nu_{22}(k)-v_{22}(0)\right)}\right. \\
& \left.-\frac{a_{21}}{1+a_{21} \theta^{2} c_{1} c_{2}\left(v_{21}(k)-v_{21}(0)\right)}\right] \text {. }
\end{aligned}
$$

Using relations (104), (149), and (161) and passing to the limit for $k \rightarrow 0$, we can obtain the dependence of partial fluxes of the components on the pressure, temperature, and composition of the mixture. For $k \neq 0$, expressions (144) and (160) enable us to analyze the relaxation mechanism of the emerging density fluctuation with a characteristic size of $r \approx$ $2 \pi / k$.

In order to analyze the experimentally observed partial fluxes, it is convenient to pass to the coordinate representation. Spectra (160) have the real and imaginary parts since pair correlation function $v_{i j}(k)$ is a complex quantity in accordance with relations (120) and (125). Separating the real and imaginary parts of the spectra and applying the inverse Fourier transformation to expression (149), we obtain

$$
\begin{aligned}
& j_{i}=n_{i} \Psi_{i}-D_{i} \frac{\partial n_{i}}{\partial x}, \\
& \Psi_{i}=\operatorname{Re} \omega_{i}\left(k \longrightarrow \frac{2 \pi}{L}\right), \\
& D_{i}=\left.\operatorname{Im} \frac{\omega_{i}(k)}{k^{2}}\right|_{k \rightarrow 2 \pi / L} .
\end{aligned}
$$

Here $D_{i}$ is the diffusion coefficient of a component, $L$ is the channel length, and $\Psi$ is the term emerging due to the mutual effect on the mixture components in a channel for large fill factors. It should be noted that, since $L \gg r_{c}$, where $r_{c}$ is the characteristic size of clusters, passing to the limit for $k \rightarrow 2 \pi / L$ in relation (161) corresponds to averaging over characteristic scales of inhomogeneities in the case when the ground state of the system is clustered. Separating the terms linear in the wave vector from the real part of the spectrum, we obtain the following expression for $\Psi$ :

$$
\Psi_{i}=-D_{i} \frac{1}{T} \frac{\partial U_{i}}{\partial x}+V_{i}
$$

Here the first term describes the transport of molecules of the $i$ th component, which is induced by the effective intermolecular interaction, while the second term has the meaning of the "drag" effect familiar in the kinetics of mixtures. Substituting
(162) into (161), we obtain the final expression for partial fluxes:

$$
j_{i}=-D_{i} \frac{\partial n_{i}}{\partial x}-D_{i} \frac{n_{i}}{T} \frac{\partial U_{i}}{\partial x}+V_{i} n_{i}
$$

Thus, it follows from this equation that the partial flux is the sum of three terms. The first term corresponds to diffusion transport, while the second term emerges due to field diffusion. These two terms in the $k$ representation can be combined into one by introducing the effective diffusion coefficient

$$
D_{i}=D_{i}^{(0)}\left(1+\frac{n_{i}}{T} U(k)\right)
$$

It was shown in the previous section that, for large filling factors, clusters are formed in the system. The mechanism of density relaxation may be different for the case of transport over the characteristic scale $L$, when $2 \pi \sigma_{\text {av }} / L \ll 1$ and $k \rightarrow 0$ (which corresponds to macroscopic transport of gas components through the channel), and for the case when $k \neq 0$, which corresponds to relaxation over distances comparable to the size of clusters formed in the system. To prove this, we must analyze dependences $\Delta v_{i j}(k)$. In order to avoid cumbersome formulas, we consider this problem in greater detail in the limiting case of $c_{1}=1$, which corresponds to a one-component mixture.

Passing to the limit for $c_{1} \rightarrow 1$ in (160), we obtain the relaxation frequency spectrum for a one-component system; it is easy to obtain (70). It should be noted that, in accordance with the relation (70), the pair distribution and, hence, the pair correlation function associated with it depend not on the wave vector $k$, but on the product $i k$ :

$$
\theta \nu(k) \equiv \theta \nu(i k) .
$$

Let us now write relation (164) in the form

$$
\begin{aligned}
\omega(k) & =-\frac{i D_{0} k^{2}}{1+\theta \nu(0)+\theta \nu(k)-\theta \nu(0)}=-\frac{i D_{0} k^{2}}{1+\theta \nu(0)+\theta \Delta \nu} \\
& =-\frac{i D_{0} k^{2}}{1+\theta \nu(0)+\theta \operatorname{Re} \Delta \nu+\theta i \operatorname{Im} \Delta \nu} .
\end{aligned}
$$

Separating the real and imaginary parts from relation (165), we obtain

$$
\begin{aligned}
\omega(k)= & -\frac{i D_{0} k^{2}\left((1-\theta)^{2}+\theta \operatorname{Re} \Delta \nu\right)}{\left((1-\theta)^{2}+\theta \operatorname{Re} \Delta v\right)^{2}+(\theta \operatorname{Im} \Delta v)^{2}} \\
& -\frac{D_{0} k^{2} \theta \operatorname{Im} \Delta v}{\left((1-\theta)^{2}+\theta \operatorname{Re} \Delta \nu\right)^{2}+(\theta \operatorname{Im} \Delta v)^{2}}
\end{aligned}
$$

Here we have used the fact that

$$
1+\theta \nu(0) \approx(1-\theta)^{2} .
$$

This relation follows from the known relation [46]

$$
\frac{\partial p}{\partial \theta} \simeq \frac{1}{1+\theta \nu(0)}
$$


describing the increase in the compressibility with density. Using relation (165), we now expand $\Delta v$ to within first-order terms:

$$
\Delta v=\left.\frac{\partial \theta \nu(k)}{\partial(i k)}\right|_{k=0} i k \equiv i \theta v^{\prime}(0) k
$$

The value of $v^{\prime}(0)$ is finite for $\theta \neq 0$. For example, using direct expansion into the Taylor series, we can obtain

$$
\theta \nu^{\prime}(0)=-\theta+\frac{4}{3} \theta^{2}-\frac{1}{2} \theta^{3}
$$

Substituting relation (170) into (167), we get

$$
\omega(k) \approx-\frac{i D_{0} k^{2}(1-\theta)^{2}}{(1-\theta)^{4}+\left(\theta \nu^{\prime}(0)\right)^{2} k^{2}}-\frac{D_{0} k^{3} \theta \nu^{\prime}(0)}{(1-\theta)^{4}+\left(\theta \nu^{\prime}(0)\right)^{2} k^{2}} .
$$

To calculate the fluxes for high densities $(\theta \sim 1)$, we must consider two limiting transitions in relation (172): for $\theta \rightarrow 1$ and $k \rightarrow 0$. From the standpoint of physics, the transition for $\theta \rightarrow 1$ and $k \neq 0$ corresponds to the analysis of transport in a dense cluster. The transition for $k \rightarrow 0$ and arbitrary $\theta$ is equivalent to analysis of diffusion in a channel of length $L \gg r_{c}$, where $r_{c}$ is the characteristic size of a cluster. For $\theta \rightarrow 1$, we obtain, instead of relation (172),

$$
\omega(k)=-\frac{D_{0} k}{\theta \nu^{\prime}(0)} .
$$

This relation shows that spectrum $\omega(k)$ corresponds to the hydrodynamic mode [46], for which quantity $D_{0} / \nu^{\prime}(0)$ is the effective velocity of sound.

Passing in relation (172) to the limit for $k \rightarrow 0$ and retaining the lowest order in wave number $k$, we obtain

$$
\omega(k)=-\frac{i D_{0} k^{2}}{(1-\theta)^{2}} .
$$

This relation shows that the spectrum in this case is of the diffusion type with the diffusion coefficient

$$
D \approx \frac{D_{0}}{(1-\theta)^{2}}
$$

which increases indefinitely as $\theta \rightarrow 1$.

It should be noted that, in accordance with (172), both relaxation mechanisms (hydrodynamic and diffusion) operate in the system when $k \neq 0$ and $\theta \neq 1$. From the standpoint of physics, this corresponds to the diffusion transport between clusters (diffusion mode), over which density perturbations propagate (hydrodynamic mode). In spite of different interpretations of the sequence of limiting transitions, the calculated dependences of fluxes on external conditions are found to be equivalent from the physics standpoint. This is due to the fact that the hydrodynamic component is manifested in the second case in the increase in the effective diffusion coefficient $D \approx D_{0} /(1-\theta)^{2}$ for high values of fill factor $\theta$.

In the two-component case, the transition for $k \rightarrow 0$ corresponds to the transition from relation (160) to (153).
It can be seen from relation (153) that the obtained spectra are of the diffusion type since $\omega_{j}^{(0)}=-i D_{j} k^{2}, j=1,2$, while quantities $a_{i j}$ and $b_{i j}$ are independent of $k$. The limiting transition for $\theta \rightarrow 1$ in the two-component case involves considerable computational difficulties. However, the above analysis shows that the relaxation frequency spectra for the components also acquire a hydrodynamic mode in view of the dependence of pair correlation function (120) on factor $i k$ for $\theta \rightarrow 1$.

Depending on the sequence of limiting transition, a decisive role in relation (163) is played either by the term corresponding to diffusion or the nongradient (hydrodynamic) part of the flux. Since the gas flux for arbitrary fill factors is measured in experiments on the penetrability of the membrane over large distance $\left(L \gg r_{c}, k \rightarrow 0\right)$, the limiting transition for $k \rightarrow 0$ should be performed first. In this case, the effects associated with the formation of clusters are taken into account in the dependence of the diffusion coefficient on the concentration and fill factor of the channel. Numerical calculations show that the last term in relation (160) is insignificant in the entire range of concentrations and fill factors of the channel. Thus, the relaxation frequency spectrum of a two-component system can be treated as a diffusion spectrum in the entire range of concentrations and fill factors, where the role of diffusion coefficient is played by a quantity taking into account clustering of the components in the channel.

It should be noted that for calculating response function (132), relaxation frequency spectra (160), and, as a consequence, partial fluxes (163), we used the fluctuationdissipative theorem (119) presuming the homogeneous ground state of the system. In the case when the ground state of the system is clustered (see Figure 13(d)), the kinetic version of the fluctuation-dissipative theorem [98] should be used:

$$
\begin{aligned}
& \left\langle\delta n(x, t) \delta n\left(x^{\prime}, t\right)\right\rangle \\
& \quad=-T \int_{0}^{\infty} \beta\left(x, x^{\prime}, t, \tau\right)\left(f(x, \tau)-f\left(x^{\prime}, \tau\right)\right) d \tau,
\end{aligned}
$$

where $f(x, \tau)$ is the probability of a particle being located at point $x$. In accordance with the detailed balancing principle, in the case when states $x$ and $x^{\prime}$ differ from the equilibrium state insignificantly, we have

$$
f\left(x^{\prime}, t\right) \sim f(x, t) \exp \left(\frac{\Delta E}{T}\right)
$$

where $\Delta E$ is the energy difference between states $x$ and $x^{\prime}$. Using this relation, we obtain the following expression for the fluctuation-dissipative theorem:

$$
\begin{aligned}
\left\langle\delta n(k, \omega) \delta n\left(k^{\prime}, \omega\right)\right\rangle \\
=-T \int d x d x^{\prime} d t \exp (i k x) \exp \left(i k^{\prime} x^{\prime}\right) \exp (i \omega t) \\
\quad \times \int_{0}^{\infty} \beta\left(x, x^{\prime}, t, \tau\right) \exp \left(\frac{\Delta E}{T}\right) d \tau .
\end{aligned}
$$


Applying the Fourier transformation to this relation and using (151), we obtain the relaxation frequency spectrum in the form

$$
\omega^{(\text {new) }}(k)=\omega(k) \exp \left(-\frac{\Delta E}{T}\right)
$$

Thus, the application of relation (176) instead of (132) leads to the emergence of an additional exponential term in relation (163):

$$
j_{i}(k, t)=\frac{n_{i}(k, t)}{k} \omega_{i}(k) \exp \left(-\frac{\Delta E}{T}\right)
$$

where $\Delta E$ is the depth of the potential well occupied by the system. In the case of small fill factors of the channel, the state of the system is homogeneous and $\Delta E=0$ (see Figure 13(a)). Then relation (180) transforms into (163). As the fill factor of the channel increases, the state of the system becomes clustered. For $\Delta E \ll T$, the exponential in relation (180) can be disregarded, and the flux virtually coincides with the flux calculated for a homogeneous state. This is due to the fact that expression (142) for the lifetime of clusters practically coincides with expression (141) for the lifetime of clusters in a homogeneous state. In this case, as noted previously, density excitation propagates via a cluster, leading to an increase in the effective diffusion coefficient. A further increase in the fill factor of the channel increases the value of $\Delta E$. The flux decreases thereby, which can be explained by an increase in the lifetime of the clusters formed in the channel. As a result, the mechanism of excitation transport via a cluster is not realized.

Thus, for small fill factors of the channel, the transport in the system follows the diffusion mode. This can be demonstrated by passing to the limit of small fill factors $\theta$ in relation (160). Then the second term in the denominator becomes insignificant and the spectra are reduced to diffusion spectra (153). An increase in the fill factor results in the formation of clusters. It was shown in the previous section that, depending on the fill factor of the channel, the clusters formed may be either short lived or stable in the case when the ground state of the system is clustered. For short-lived clusters (see Figure 13(b)), the transport between clusters occurs through diffusion, while the transport over clusters occurs through a rapid barrier-free transfer of density excitation, and the arrival of a particle from one side of a cluster leads to the emergence of a particle from the other side [100]. In this case, the spectra contain both the diffusion and the hydrodynamic modes, and the effective diffusion coefficient increases in accordance with (175). An increase in the fill factor of the channel reduces the distance between clusters and increases their size. Since the transport over a cluster is faster than the diffusion transport, the effective diffusion coefficient and the partial flux increase. However, an increase in the fill factor also increases the lifetime of the clusters formed in the channel; as a result, the rate of excitation transfer over a cluster decreases. In the case when a cluster is stable (see Figure 13(d)), the transport of excitation over the cluster is ruled out, and the presence of such a cluster in the channel leads to blocking of transport in the system.
Using relations (160) and (180), we can calculate the partial gas fluxes proceeding from the data on the penetrability for pure components. Figures 17 (a) and 17(b) show the dependence of partial fluxes on the mixture composition in the case when both gases are slightly adsorbed. The energies of interaction and the diameters of particles correspond to a methane-argon gas mixture $\left(a=3.8 \AA, \sigma_{1}=3.6 \AA, \sigma_{2}=\right.$ $3.0 \AA, \varepsilon_{1}=0.38 \mathrm{eV}, \varepsilon_{2}=0.25 \mathrm{eV}[51], p=100 \mathrm{kPa}$, and $T=300 \mathrm{~K})$.

It can be seen from Figure 17(a) that an increase in the concentration of the first component for fixed pressure and temperature reduces the filling factor with the second component, while the filling factor with the first component increases thereby. A decrease in the total fill factor of the channel in the concentration range $0<c<0.4$ of first component takes place due to depletion of the channel in the second component (Figure 17(b)). An increase in the total fill factor of the channel for $c>0.4$ is associated with preferred enrichment of the channel in the first component (see Figure 17(b)). The partial flux of the first component in this case increases monotonically due to an increase in the filling factor with the first component (see Figure 17(a)), while the partial flux of the second component decreases due to a decrease in the filling factor with the second component. The transport is of the diffusion type, and clusters are not formed in the channel in view of small fill factors of the channel $(\theta \leq 0.25$ for any composition of the mixture).

Figures $17(\mathrm{c})$ and $17(\mathrm{~d})$ show the dependence of partial fluxes on the mixture composition in the case when one of the gases (the first) is a strong sorbate, while the other gas is a weak sorbate. The energy of interaction and the diameter of particles correspond to a butane-methane gas mixture $\left(a=3.8 \AA, \sigma_{1}=4.3 \AA, \sigma_{2}=3.6 \AA, \varepsilon_{1}=0.61 \mathrm{eV}\right.$, $\varepsilon_{2}=0.38 \mathrm{eV}$ [51], $p=2000 \mathrm{kPa}$, and $\left.T=300 \mathrm{~K}\right)$. The behavior of the total and partial fill factors of the channel upon an increase in the concentration of the first (highly adsorbed) component in this case coincides qualitatively with the case of slightly adsorbed gases described previously: the partial filling factor with the first (highly adsorbed) component increases monotonically against the background of a monotonic decrease in the filling factor with the second (slightly adsorbed) component (see Figure 17(c)).

The behavior of partial fluxes in this case is less trivial. It can be seen from the figure that the partial flux of the first component increases in the concentration interval $0<c<0.2$. This is due to the fact that, in accordance with relation (141), short-lived clusters (see Figure 13(a)) whose size increases with concentration are formed for such concentrations and fill factors of the channel. The transport over clusters occurs via a barrier-free transfer of density excitation, which increases the effective diffusion coefficient. This process is similar to the transport in one-component systems for high fill factors (see Section 2.1). It should be noted, however, that the formation of clusters and the mechanism of transfer of density excitation through a cluster in a twocomponent mixture can be realized for smaller fill factors $(\theta=0.71$; see Figures $13(\mathrm{~b}), 17(\mathrm{c})$, and $17(\mathrm{~d}))$ as compared to the one-component case $(\theta \geq 0.8)$. An increase in the concentration of the first component increases the lifetime 


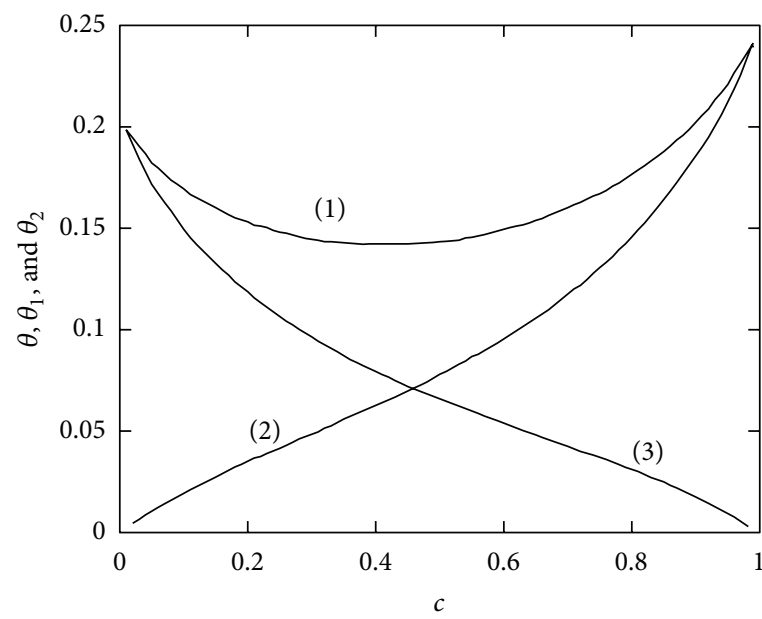

(a)

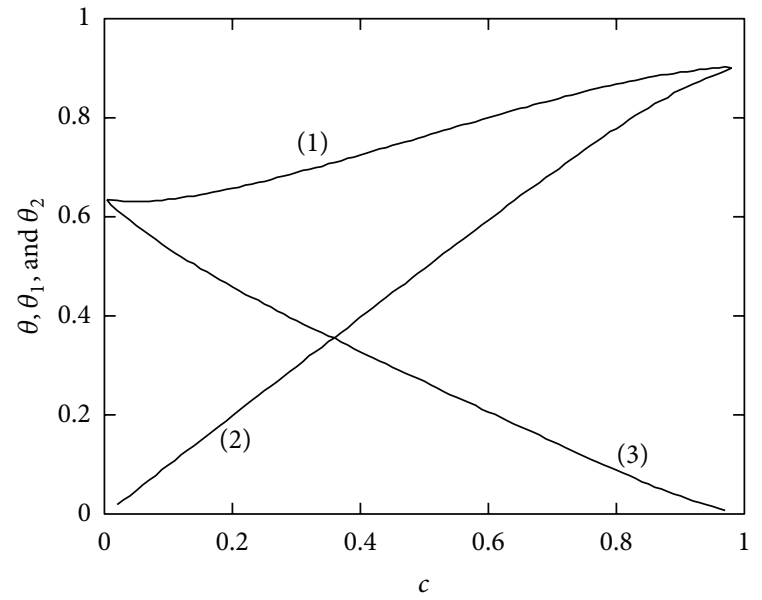

(c)

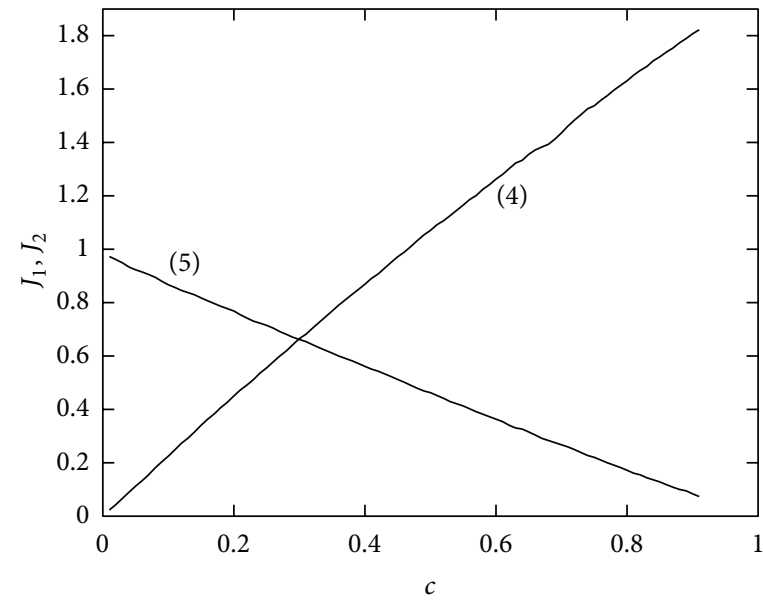

(b)

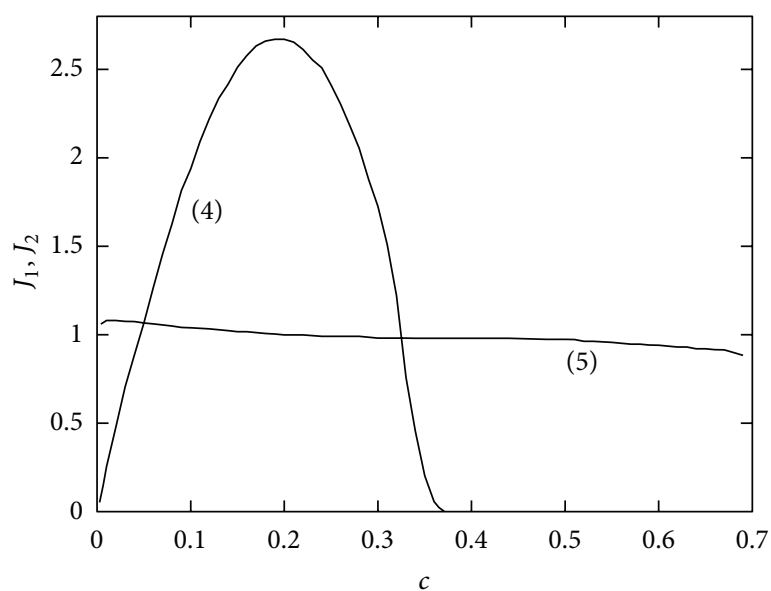

(d)

Figure 17: Dependence of the total and partial fill factors of a channel (a, c) and partial fluxes (b, d) on the composition of methane-argon ( $a, b)$ and butane-ethane mixtures (c, d): (1) - total fill factor of a channel; (2), (3) - filling factors with the first and second components, respectively; (4), (5)-partial fluxes of the first and second components, respectively; $c$ is the concentration of the first component in the mixture.

of the clusters formed in the system (see Figures 14(b) and $15(\mathrm{c}))$. As a result of this increase, the transport over clusters becomes slower and the flux decreases. A further increase in the concentration leads to the formation of stable clusters in the channel (see Figures 14(c) and 16(c)); in accordance with relation (120), the lifetime of these clusters is much longer than the lifetime (141) of short-lived clusters since $\delta F \gg T$. In this case, the transfer of density excitation over clusters is ruled out and the partial flux vanishes.

Thus, three mechanisms of particle transport in a channel are possible in the two-component case. For small fill factors, the transport occurs through diffusion. An increase in the fill factor leads to the formation of short-lived clusters in a channel. As in the one-component case, this leads to an increase in the effective diffusion coefficient due to barrierfree transport of density excitation over a cluster. As the fill factor increases further, the characteristic lifetime of clusters increases, leading to a decrease in the flux. A further increase in the fill factor makes the clusters stable, and the so-called blocking effect takes place, when the partial flux of one of the components vanishes. A decrease in pressure does not lead to a qualitative change in the behavior of the dependences of partial fluxes on the mixture composition.

2.4.3. Comparison with Experiment. The transport of twocomponent gas mixtures in zeolite membranes MFI (silicalite, ZSM-5) with a pore diameter of $\sim 0.6 \mathrm{~nm}$ has been studied by the technique widely used for one-component gases [51]. However, in contrast to the rich variety of experiments with one-component gases, a consistent analysis of a twocomponent mixture has not been reported in the literature. The most detailed information on penetrability for twocomponent mixtures is given in [51,97].

The experiments $[51,97]$ on penetration of two-component mixtures were made in a chamber with a porous stainless steel substrate with a thickness of approximately $3 \mathrm{~mm}$. A polycrystalline layer of zeolite ZSM-5 with a thickness of 
$\sim 50 \mu \mathrm{m}$ and a random orientation of crystals was deposited on the substrate. Zeolites have a complex crystalline structure based on silicon oxide with admixtures of sodium and aluminum [51]. The crystalline structure of zeolite ZSM-5 is formed by straight channels with an elliptical cross section $\left(0.57 \times 0.52 \mathrm{~nm}^{2}\right)$ intersecting sinusoidal channels with a circular cross section of diameter $0.54 \mathrm{~nm}$ (Section 2.1). The measured adsorption capacity and the calculated fraction of molecules in the channel intersections make it possible to treat the channels as one dimensional for the molecular mixtures under study (Section 2.1). The experiments [51, 97] were carried out at temperatures from 300 to $700 \mathrm{~K}$ under pressures from 25 to $500 \mathrm{kPa}$ on the external side of the membrane. The purity of the mixture components was higher than $99.95 \%$. The mixture composition at the membrane exit was measured on a quadrupole mass spectrometer with a sensitivity of approximately $25 \mathrm{ppm}$ when a Faraday cell was used for recording. The selectivity measured in the experiments was determined as the relative change in concentration $c$ of $n$-butane molecules and concentration $1-c$ of methane molecules at the membrane entrance $(F)$ and exit $(p)$ :

$$
\alpha=\left(\frac{c}{1-c}\right)_{F}:\left(\frac{c}{1-c}\right)_{p} .
$$

It should be noted that, for a constant composition of the mixture at the membrane entrance, the selectivity is proportional to the ratio of partial fluxes of the mixture components at the membrane exit:

$$
\alpha \sim \frac{J_{1}}{J_{2}} .
$$

It was found experimentally [51] that the partial flux of the highly adsorbed gas $n-\mathrm{C}_{4} \mathrm{H}_{10}$ at $T=300 \mathrm{~K}$ under a total pressure of $p=100 \mathrm{kPa}$ changes considerably (by a factor of several units) in the presence of the slightly adsorbed component $\mathrm{CH}_{4}$ as compared to the flux of a pure gas, while the partial flux of the slightly adsorbed gas changes by two orders of magnitude in the presence of the strongly absorbed component. Depending on the mixture composition, selectivity $\alpha$ varies and attains its maximum value $\alpha_{\max }=380$ for a concentration ratio of $5: 95$ at the membrane entrance. It was shown [97] that the dependences of partial fluxes on the mixture composition and external pressure for the mixtures studied $\left(\mathrm{C}_{2} \mathrm{H}_{6}-\mathrm{CH}_{4}\right.$ and $\left.\mathrm{C}_{3} \mathrm{H}_{8}-\mathrm{CH}_{4}\right)$ are monotonic in the range of experimental pressures. The partial flux of methane decreases in the presence of the second component (ethane or propane), while the partial flux of the second component increases with its concentration in the mixture. The selectivity of both mixtures increases monotonically with the concentration of the more highly adsorbed component in the mixture. The dependences of the partial fluxes on the total pressure of the mixture are also monotonic, but the pressure dependence of selectivity in ethane for the mixture $\mathrm{C}_{2} \mathrm{H}_{6}-\mathrm{CH}_{4}$ has a peak at a pressure of $p \approx 300 \mathrm{kPa}$ [97]. Similar dependences of partial fluxes were observed in experiments with another mixture of slightly adsorbed gases $\left(\mathrm{CO}_{2}-\mathrm{N}_{2}\right)$ [53] (Figure 18).

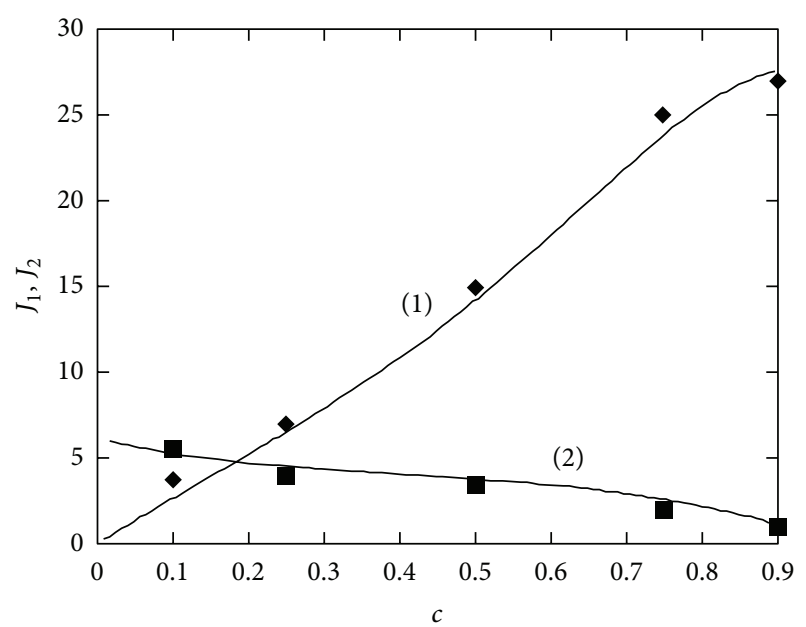

FIGURE 18: Dependence of partial fluxes on the mixture composition for slightly adsorbed gases: (1), (2)-theoretical dependences for $\mathrm{CO}_{2}$ and $\mathrm{N}_{2}$ fluxes, respectively; $c$ is the $\mathrm{CO}_{2}$ concentration above the membrane; squares correspond to experimental data from [53]; $a=3.8 \AA, \sigma_{1}=3.8 \AA$, and $\sigma_{2}=3.6 \AA[51] ; p=100 \mathrm{kPa}, T=300 \mathrm{~K}$.

The theory developed in the previous sections enables us to describe peculiarities in the transport of binary gas mixtures in zeolite membranes. In the case of a mixture of slightly adsorbed gases, when the fill factor of a channel is small $(\theta \ll 1)$ for any mixture composition, the system relaxes in accordance with the diffusion mechanism corresponding to diffusion of solitary particles without the formation of clusters in a channel. In this case, in accordance with (104), (160), and (180), the partial flux of the first component decreases monotonically upon an increase in the concentration of the second component, while the partial flux of the second component increases with its concentration in the mixture.

Figure 18 shows that the theoretical curves calculated by formulas (104), (160), and (180) are in good agreement with the experimental data borrowed from [53]. The mean distance $a$ between the seats in a channel was estimated proceeding from the value corresponding to a close packing of a unit cell of a ZSM-5 membrane with nitrogen (Section 2.1).

The filling factor for mixtures with a strongly absorbed component depends on the mixture composition; it is significant and can be as high as $\theta \sim 1$. In this case, in accordance with the arguments put forth in Section 2.4.1, the dependence of fluxes on the mixture composition is nonmonotonic due to the formation of clusters in a channel.

Figure 19 shows the dependence of the ratio of partial fluxes of gases for the mixture $n-\mathrm{C}_{4} \mathrm{H}_{10}-\mathrm{CH}_{4}$ on the butane concentration in the mixture under a pressure of $p=$ $100 \mathrm{kPa}$ at temperature $T=300 \mathrm{~K}$. It can be seen from the figure that the ratio of partial fluxes increases with the concentration of the highly adsorbed component (butane) in the concentration range $0<c<0.35$. Analysis of experimental data [51] and calculations based on formulas (104), (160), and (180) show that the methane flux for a butane concentration of $c>0.05$ changes insignificantly upon an 


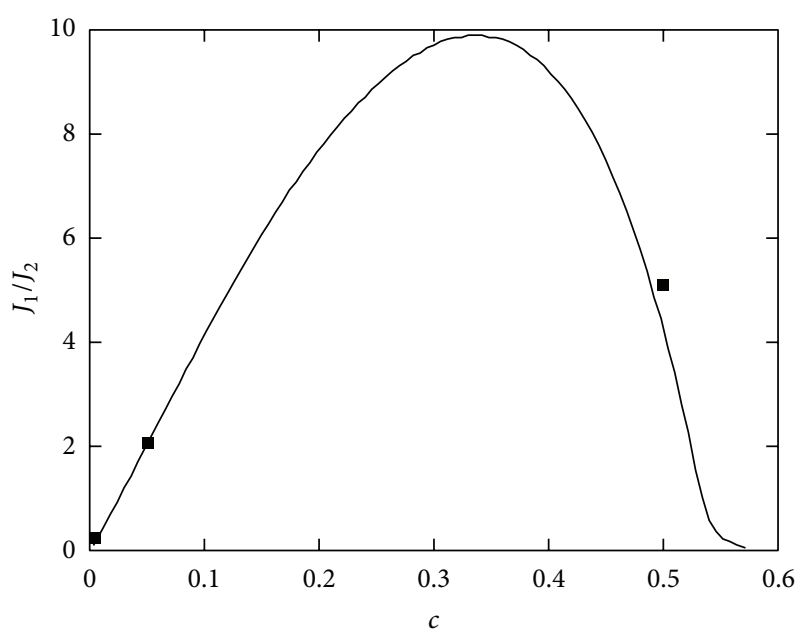

FIGURE 19: Ratio of the partial fluxes of butane and methane as a function of the butane concentration; $c$ is the concentration of $n$ $\mathrm{C}_{4} \mathrm{H}_{10}$ above the membrane; squares correspond to experimental data obtained in [51]; $a=3.8 \AA, \sigma_{1}=4.3 \AA$, and $\sigma_{2}=3.6 \AA$ [51]; $p=100 \mathrm{kPa}, T=300 \mathrm{~K}$.

increase in the butane concentration in the mixture. In this case, the behavior of the flux ratio is completely determined by the behavior of the butane flux. The increase in the butane flux is due to the fact that, in accordance with formula (141), short-lived butane clusters are formed for concentrations $0<$ $c<0.35$ and for the fill factors corresponding to these concentrations (see Figure 13(a)); the size of these clusters increases with the concentration of the highly adsorbed component. The transport over clusters (see Figure 13(b)) occurs via barrier-free transfer of density excitation, leading to an increase in the effective diffusion coefficient for butane, which is observed for $c<0.35$. The increase in the butane concentration in the mixture increases the lifetime of the clusters formed in the system (see Figures 14(b) and 15(c)). With increasing lifetime, the transport over the clusters slows down and the butane flux decreases for concentrations $c>$ 0.35. A further increase in the concentration results in the formation of stable clusters in a channel (see Figures 14(c) and 16(c)), leading to a rapid (exponential) decrease in the butane flux upon an increase in its concentration in the mixture.

Thus, it follows from the theory that the butane flux becomes exponentially small for a butane concentration of $c>0.6$ in the mixture, and the transport of particles must be blocked. It should be noted that calculations based on formulas (104), (160), and (180) for butane concentrations in the interval $0<c<0.05$ show that the methane flux decreases approximately by an order of magnitude upon an increase in the butane concentration, which is also in qualitative agreement with the experimental data [51]. The numerical difference between the predictions of the theory developed previously and the experimental data in this range of butane concentrations are due to the fact that relations (104), (160), and (180) were derived for the model potential of intermolecular interaction of the type of interaction (101) between hard spheres. It is well known that the potentials of hard spheres correctly describe the behavior of real systems for high densities $[46,101]$. In order to describe the behavior of the system in the entire range of fill factors, potentials of the Lennard-Jones type, which take into account the attraction between particles even in the zeroth order in density, should be used. However, although the application of this type of potentials of intermolecular interaction does not change the results qualitatively, it leads to the formation of butane clusters at lower filling factors and, hence, to a sharper decrease in the methane flux upon an increase in the butane concentration in the concentration range under investigation.

Relations (104), (160), (180), and (181) also make it possible to derive the dependence of the selectivity on the pressure, temperature and composition of the mixture. Figure 20(a) shows the dependence of the ethane selectivity for the $\mathrm{C}_{2} \mathrm{H}_{6}-\mathrm{CH}_{4}$ mixture with a concentration ratio of $50: 50$ at the entrance on the total pressure of the mixture [97]. It can be seen that the dependence is nonmonotonic. This is due to the fact that the diffusion coefficient first increases due to an increase in the pressure and fill factor of the channel as a result of the formation of short-lived clusters, and the partial ethane flux increases (Figure 20(b)). The increase in the total fill factor of the channel is determined by the increase in the partial fill factors for both components. This process continues until the channel filling with ethane slows down. In this case, the fill factor of the channel increases mainly due to channel filling with methane. At such pressures, the increase in the ethane flux slows down (see Figure 20(b)), while the methane flux continues to increase (Figure 20(c)). The theoretical dependences plotted in the figures show that a further increase in pressure due to an increase in the lifetime of ethane clusters formed in the channel must lead to a decrease in the ethane flux (Figure 20(b)). Thus, the analysis of experimental data proves that the theory constructed here describes the experimental results satisfactorily.

One of the main results of the proposed theory is the prediction of nonmonotonic dependences of partial fluxes and selectivities on the mixture composition and pressure (see Figures 19 and 20), while conventional models [51, 53, 97] (in particular, the generalized Maxwell-Stefan equation $[51,97])$ lead to monotonic dependences of these quantities on the same parameters (see Figure 20(a)). The qualitative discrepancy between the dependences in question is due to the fact that conventional models $[51,53,97]$ take into account only the finite size of particles, in which the interaction between particles in a channel is disregarded. It is well known $[46,47,101]$, however, that the interaction between particles in dense systems plays a decisive role both in the construction of the equations of state of the system and in the description of transport. For example, the inclusion of interaction between particles of the hard-sphere type leads to the emergence of peaks in the pair correlation function at distances equal to one, two, three, and so forth particle diameters $[44,46$, 101]. This fact indicates the existence of "effective" attraction between particles and necessitates the inclusion of cluster formation in the description of the behavior of the system at high densities, when it passes to a spatially inhomogeneous state. It was demonstrated previously that this leads to the emergence of nonmonotonic dependences of fluxes and selectivities on external parameters. 


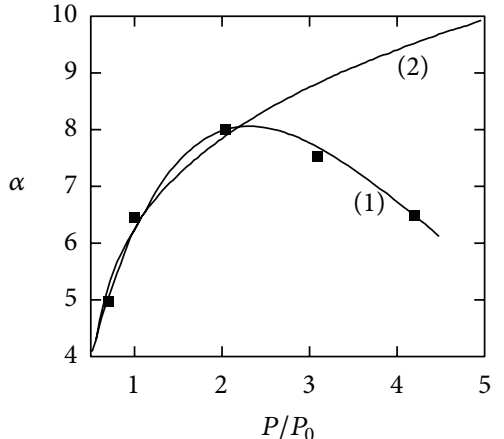

(a)

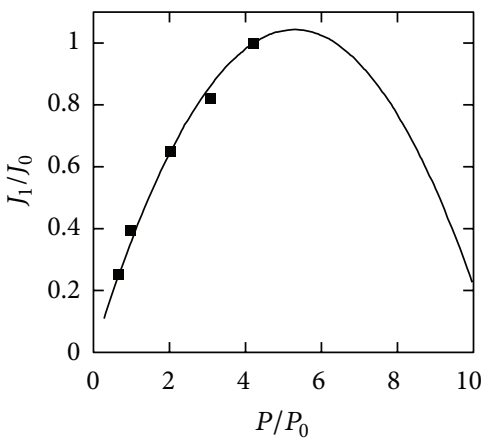

(b)

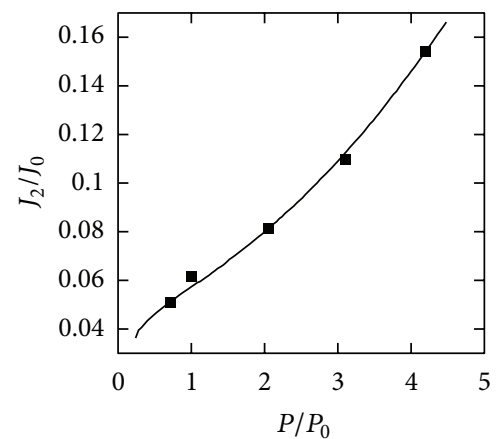

(c)

Figure 20: Dependence of the ethane selectivity (a) and of the partial fluxes of $\mathrm{C}_{2} \mathrm{H}_{6}$ (b) and $\mathrm{CH}_{4}$ (c) for the $\mathrm{C}_{2} \mathrm{H}_{6}-\mathrm{CH}_{4}$ mixture on the total pressure of the mixture: (1)—calculations based on formulas (104), (160), and (180) and (181); (2)—calculations based on the generalized Maxwell-Stefan equation [97]; squares correspond to experimental data obtained in [51]; $a=3.8 \AA \sigma_{1}=3.8 \AA$, and $\sigma_{2}=3.6 \AA$ [51]; $J_{0}=$ $63 \mathrm{mmole} /\left(\mathrm{m}^{2} \mathrm{~s}\right), p_{0}=100 \mathrm{kPa}$.

It should be noted that the stabilization of clusters in $1 \mathrm{D}$ systems by the second component at high fill factors, which has been investigated here, was obtained numerically in a recent work [83]. It should be borne in mind, however, that, in accordance with our previous results (Section 2.1), clusters with a large but finite lifetime can be formed even in a onecomponent system with high filling factors. It is $1 \mathrm{D}$ gold clusters of this type that were apparently observed in recent experiments under the ultrahigh vacuum conditions.

\section{Results and Discussion}

The analysis of the experimental data showed that for most gases investigated, the sorption and transport can be treated as a process in a one-dimensional (1D) system. In statistical physics, the theoretical models of $1 \mathrm{D}$ systems are classical examples of exactly solvable models [44]. It is well known [34, $46]$ that in $1 \mathrm{D}$ systems, a phase transition (condensation) does not occur as density increases. This means that, specifically, no critical nuclei are present in the system, and stable nuclei of a new phase with macroscopically long lifetimes do not appear in the system. At the same time, the system of $1 \mathrm{D}$ channels in zeolite membranes consists of channels of finite length, where for a sufficiently high filling factors, clusters with sizes comparable to the channel length can form. Thus, the description of molecular transport in zeolite membranes reduces to describing transport in a $1 \mathrm{D}$ system, where there are strong density fluctuations with a finite lifetime of clusters. The density functional method [38] makes it possible to calculate the spectrum of density fluctuations and the diffusion coefficient for particles with arbitrary density and arbitrary laws of interaction with one another and with the channel walls. Since the flux measured in the experiments performed in [41] is determined by the diffusion coefficient and the filling factor of the channels in the membrane, the sorption isotherm of a one-component molecular gas is calculated separately. For the one-dimensional channels considered in the present paper, this problem is solved exactly for an arbitrary intermolecular interaction potential. A new feature here is an analysis of the dependence of the lifetime and size of the 1D clusters on the filling factor of the channel with particles. A description of molecular transport for an arbitrary filling factor $(\theta)$ of a channel is performed. It was found that as the filling factor approaches 1 , so that blocking of the relative motion of the particles becomes substantial, the diffusion coefficient increases. Following [31], a new diffusion mechanism is observed. It makes it possible to explain the transition from activated diffusion of single particles in a channel with low filling factors to collective fast barrier-free diffusion, which consists of the propagation of density disturbances for large values of $\varepsilon_{0}$. In the hardsphere approximation, as $\bar{N}_{1}$ increases, the well-known [46] effective attraction of the molecules-hard spheres-results in vanishing of the energy barrier for diffusion along the channel axis. Another consequence of the effective attraction of molecules is the formation of molecular clusters in the channel, which have a finite lifetime because of the onedimensionality of the system. The size and lifetime of the clusters increase as $\theta$ increases. The diffusion of particles in clusters is described as a barrier-free process of propagation of density disturbances. The pressure, temperature, and filling factor dependences of the flux of molecules make it possible, even in the high-sphere model, to describe the experimental data currently known to the authors.

We have shown that contradictory experimental data, reporting the presence of the SFD mode and transport acceleration with the increasing density, can be described within the proposed model consistently accounting for correlation effects in a strongly fluctuating $1 \mathrm{D}$ system of identical particles. The molecular transport in a 1D system can change with time going from the Einstein diffusion mode to SFD mode and then again to a normal diffusion at large observation times. Whereas the sequence of these modes is general, their actual realization depends on the concrete characteristics of the system. For example, at very low densities one expects to observe only a normal diffusion mode. If we consider a system of weakly interacting particles $(u \leq 1)$, we can see that at low densities $\theta \leq 0.2$ and time scale $t<l_{0}^{2} / D_{0}$, the particles 
move independently and MSD obeys the Einstein law $\left\langle x^{2}\right\rangle \sim$ $t$. At times $t>l_{0}^{2} / D_{0}$, when a particle mobility is limited by other particles, the SFD diffusion mode $\left(\left\langle x^{2}\right\rangle \sim t^{1 / 2}\right)$ should appear. As the density is increased, collective effects force the $1 \mathrm{D}$ system to transform into a nonhomogeneous state, characterized by a formation of clusters with a finite lifetime [31]. In such a clustered system, a particle moves in a limited space between the clusters and at collision, it transfers its momentum to a cluster. This transfer of momentum can cause the cluster to emit a particle from the opposite side to the impact. For a system of identical particles, such a process, which we call collective diffusion $(\mathrm{CD})$, results in an increase of the diffusion coefficient with increasing density [31]. Therefore at long times $(t \rightarrow \infty)$ MSD again obeys the Einstein law $\left\langle x^{2}\right\rangle \sim t$ with an effective diffusion coefficient $D_{u}(\theta) \sim D_{0} /(1-\theta)^{2}$. We suggest that further experimental investigations could confirm the reappearance of the Einstein-like diffusion mode at high densities, which is characterized by a diffusion coefficient increasing with density. When the interaction between the particles in the 1D system is strong $(u \gg 1)$, cluster formation occurs even at low filling factors $(\theta \leq 0.2)$; one has the same general physical picture but the transitions from one diffusion mode to the other take place at lower densities.

In this paper, we also analyse the transport of a twocomponent gas mixture in subnanometer channels theoretically for an arbitrary filling factor. The main problem in this case is to take into account consistently the density effects associated with both the interaction and the finite size of the particles. This is done in the hard-sphere model, in which the interaction is manifested as an effective (dynamic) attraction of particles, leading to their correlation [46]. It is well known $[46,101]$ that, applying this model to 3D system, one can describe the density effects qualitatively and even quantitatively in some cases. The comparison with experimental data with the obtained theoretical dependences demonstrates the possibility of a quantitative description of diffusion of a two-component mixture in a $1 \mathrm{D}$ system.

The adsorption isotherm of a mixture in 1D channels is calculated; this isotherm relates the fill factor and the concentration of particles in channels to the temperature, pressure, and composition of a mixture of gases whose diffusion is studied in experiments [51, 53, 97]. The ground state of a mixture of particles in $1 \mathrm{D}$ channels is analyzed by the density functional method [38] generalized to the case of a two-component mixture. However, in contrast to [38], the free energy is obtained by calculating directly the correlation function and the response function using the method developed for $1 \mathrm{D}$ systems [45]. The density functional method is used to derive, from microscopic considerations, the equation of motion for the order parameter of the system, which is the Fourier component of the deviation of the particle concentration from its mean value. Analysis of this equation shows that the two-component mixture in channels is transformed to a spatially inhomogeneous state upon an increase in $\theta$. As a result, short-lived clusters appear in channels, with the cluster size and lifetime increasing with $\theta$. A new and unexpected result obtained in this case is the emergence of a minimum in the fluctuating part of the free energy as a function of the wave vector and the Fourier component of the order parameter. Thus, at high filling levels, the two-component mixture acquires a new property: clusters of a definite size are stabilized by a potential barrier due to the emergence of effective attraction between particles in the channels. Such a situation is typical of transitions of the system to an inhomogeneous state [99]. The lifetime of clusters formed increases exponentially in accordance with the Arrhenius law; at a low temperature, channels with such clusters might be blocked for the transportation of particles forming the mixture. It should be noted that the idea of stabilizing the clusters of one component by the other component in $1 \mathrm{D}$ systems was put forth in [83]. Thus, the description of transport in nanochannels is reduced to the description of diffusion in a spatially inhomogeneous highdensity one-dimensional system.

For a weakly nonequilibrium system, the problem of computing fluxes in $1 \mathrm{D}$ channel is reduced to the calculation of relaxation frequency spectra for density fluctuations of mixture components. Spectra $\omega_{i}(k)$ were determined by using the response functions derived in Section 2.4.1. Analysis of the dependences of $\omega_{i}$ on $k$ and on fill factor $\theta$ proved that a hydrodynamic spectrum $\omega_{i}(k)=c_{i} k$ is typical of clusters $(\theta \sim$ 1 , finite values of $k$ ), while for transport of excitations over distances much longer than the characteristic size of clusters $(k \ll 1)$, the spectrum is of the diffusion type, $\omega_{i}(k) \propto$ $D_{i} k^{2}$, for an arbitrary fill factor. In accordance with the dependences of spectra and fluxes on the fill factor obtained here, three regimes of particle transport can be singled out. For $\theta \ll 1$, we have diffusion of solitary particles. As the value of $\theta$ increases, the flux and the diffusion coefficient increase due to the barrier-free (hydrodynamic) transport of particles along the increasing part of the length of a $1 \mathrm{D}$ channel filled with short-lived clusters formed in it. As the value of $\theta$ increases further and the potential barrier $\left(E_{i}\right)$ stabilizing the clusters arises, particle fluxes decrease exponentially since the value of $E_{i}$ increases with $\theta$. Thus, an increase in the fill factor gives rise to the new property of $1 \mathrm{D}$ two-component systems (transport blocking by the clusters formed). A comparison of the theory with experimental data and discussion of results was carried out (Section 2.4.3). The obtained dependences of the fluxes on the mixture composition (fill factor) and of the selectivity on pressure successfully describe the experimental data known to the authors.

We have also considered an infinite quasi-1D system and showed by means of statistical methods [102] that in spite of the absence of phase transitions in an infinite quasi-1D system it can be in two states due to collective effects [33]. These two states of a quasi-1D system are characterized by the formation of clusters with finite lifetimes but different structural properties [33]. Clusters in a quasi-1D system can be interpreted as density fluctuations that can exhibit macroscopic lifetimes. As a concrete example, we estimate the lifetime, interatomic distances for $1 \mathrm{D}$ gold clusters and compare them to the available experimental and theoretical data on monoatomic gold chains. Depending on the density of the quasi-1D gold system, clusters with either large 
$(3.5 \div 4.0 \AA)$ or smaller Au-Au separations $(2.5 \div 2.9 \AA)$ can have an observable lifetime. Also we have estimated the minimal number of atoms in a 1D chain and a force required to break a chain with a minimal number of atoms in it for different metals ( $\mathrm{Au}, \mathrm{Ag}, \mathrm{Ni}$, and $\mathrm{Pt}$ ). Thus we show that the methods of statistical mechanics, in some cases, can be used to investigate the properties of finite systems. In particular, these methods allow one to explain experimentally observed anomalously large interatomic distances in monoatomic $\mathrm{Au}$ chains and to predict its maximal length and to estimate its lifetime. The described approach was applied to analyze the interatomic distances and lifetimes of quasi-1D structures of different materials such as Ag, Ni, and Pt. The obtained results successfully describe the experimental data.

\section{Conclusions}

The paper is devoted to the analysis of the correlation effects and manifestations of general properties of $1 \mathrm{D}$ systems (spatial heterogeneity that is associated with strong density fluctuations, the lack of phase transitions, the presence of frozen disorder, confinement, and blocked movement of nuclear particle by its neighbours) in nonequilibrium phenomena by considering the four examples. The anomalous transport in zeolite channels is considered. The mechanism of the transport in carbon nanotubes and MOF structures, relaxation, mechanical properties, and stability of nonequilibrium states of free chains of metal atoms, non-Einstein atomic mobility in 1D atomic systems is considered.

Description of molecular transport in zeolite membranes is reduced to the description of transport in $1 \mathrm{D}$ system in which strong density fluctuations with a finite lifetime of the clusters are presented. Response function method for density functional allows us to calculate the spectrum of density fluctuations and the diffusion coefficient of the particle with arbitrary density and arbitrary laws of interaction between particles and between a particle and a wall of the channel. Systematic account of correlation effects leads to the following physical picture of transport and mobility in strong fluctuating $1 \mathrm{D}$ confinement system.

Thus, if the interaction between particles in the $1 \mathrm{D}$ system is low $(u \leq 1)$, at low densities $\theta \leq 0.1$ at times $t<l_{0}^{2} / D_{0}$ particles move independently of each other, and, therefore, their mobility is of the Einstein type $\left\langle x^{2}\right\rangle \sim t$. At the times $t>$ $l_{0}^{2} / D_{0}$, the motion of the particles is limited due to blocking by other particles that results in the SFD regime $\left\langle x^{2}\right\rangle \sim t^{1 / 2}$. 1D system becomes spatially inhomogeneous due to collective effects in it by increasing of density of system. This state is characterized by the presence of clusters with a finite lifetime. In such a system, the diffusion of a particle is the result of its moving in a confined space between clusters with subsequent transfer of momentum to a cluster. This effect results in a disintegration of a cluster and a disconnection of the particles from the other side of the cluster. For identical particles, this process is a result of diffusion due to the "excitation" transfer along the chain of closely spaced particles and leads to an increase of a diffusion coefficient with increasing density of particles in $1 \mathrm{D}$ clustered system.
A further increase of the filling factor leads to the increase of a cluster lifetime, to the simultaneous decrease in the average distance between these clusters, and to the increase of diffusion coefficient. In this case, the dependence of the mean square displacement of particles at the large times $(t \rightarrow \infty)$ is of the Einstein type with an increased diffusion coefficient $D(\theta) \sim D_{0} /(1-\theta)^{2}, \theta \sim 1$. If the pair interaction between particles is large, the cluster formation occurs at low densities, so that all the discussed above becomes true for small filling factors zeolite channels also.

Cluster of atomic particles that forms in $1 \mathrm{D}$ systems allows to describe and explain the phenomena observed in experiments, such as the formation of atomic chains with two different interatomic distances, mechanical properties, and stability of the nonequilibrium free chains, and allows to calculate the minimal number of atomic particles of different metals in free chains. In this case, it is not necessary to use impurity atoms, the presence of which requires experimental confirmation in the chain to explain the large interatomic distances in the $1 \mathrm{D}$ chains of gold.

The description of the practically important transport of two-component mixture in membranes with subnanometer channels by the response function method allowed to show that in $1 \mathrm{D}$ system of two types of particles at high filling factors, a phase transition in an inhomogeneous state with the formation of clusters of a finite size components may occur due to an effective particle attraction.

In the framework of the response function method, the equation of motion of the order parameter of the system is derived. The order parameter is the Fourier component of the density fluctuations. Analysis of this equation shows that two-component mixture in the channels passes into a spatially inhomogeneous state with the increase of $\theta$ that results in formation of a short-lived clusters, the size and the lifetime of which increase with increasing of $\theta$.

A new result here was the occurrence of a minimum of a dependence of fluctuation part of the free energy on the wave vector and the Fourier component of the order parameter. Thus, at high filling factors in the two-component mixture, there is a new feature: the clusters of a certain size in the channels stabilizes by a potential barrier due to the effective attraction of particles.

Such a situation is typical for the transition to an inhomogeneous state. The lifetime of the clusters increases exponentially according to the Arrhenius law, and the clusters can be locked for transport of the particles of the mixture at sufficiently low temperature channels. The observed increase of the separation factor with conductivity of $1 \mathrm{D}$ channels in case of blocking a channel by stable clusters with a certain concentration is described. The dependence of the separation coefficient on the concentration, temperature, and density is defined.

A comparison of the theory with experimental data and results is discussed. The resulting fluxes depending on the composition of the mixture (the filling factor) and selectivity depending on pressure describe the experimental data presented in the literature. 


\section{Acknowledgments}

The study was supported by The Ministry of Education and Science of Russian Federation, Project no. 16.740.11.0536 and state Grants no. 2.6310.2011 and no. 2.5466.2011.

\section{References}

[1] A. Kumar, A. Kumar, and P. K. Ahluwalia, "Ab initio study of structural, electronic and dielectric properties of free standing ultrathin nanowires of noble metals," Physica E, vol. 46, pp. 259269, 2012.

[2] P. Makk, D. Tomaszewski, J. Martinek et al., "Correlation analysis of atomic and single-molecule junction conductance," ACS Nano, vol. 6, no. 4, pp. 3411-3423, 2012.

[3] K. Tsysar, D. Bazhanov, A. Saletsky, O. Brovko, and V. Stepanyuk, "Influence of hydrogen impurities on atomic and electronic structure of palladium nanowires and nanocontacts," Physical Review B, vol. 84, no. 8, Article ID 085457, 8 pages, 2011.

[4] D. Çakır and O. Gülseren, "Effect of impurities on the mechanical and electronic properties of $\mathrm{Au}, \mathrm{Ag}$, and $\mathrm{Cu}$ monatomic chain nanowires," Physical Review B, vol. 84 , no. 8, Article ID 085450, 10 pages, 2011.

[5] E. H. Huisman, M. L. Trouwborst, F. L. Bakker, B. J. van Wees, and S. J. van der Molen, "The mechanical response of lithographically defined break junctions," Journal of Applied Physics, vol. 109, no. 10, Article ID 104305, 7 pages, 2011.

[6] P. Vélez, S. A. Dassie, and E. P. M. Leiva, "Kinetic model for the long term stability of contaminated monoatomic nanowires," Physical Review B, vol. 81, no. 12, Article ID 125440, 2010.

[7] M. Zelený, M. Šob, and J. Hafner, "Ab initio density functional calculations of ferromagnetism in low-dimensional nanostructures: from nanowires to nanorods," Physical Review B, vol. 79, no. 13, Article ID 134421, 10 pages, 2009.

[8] T. Kizuka and K. Monna, "Atomic configuration, conductance, and tensile force of platinum wires of single-atom width," Physical Review B, vol. 80, no. 20, Article ID 205406, 2009.

[9] A. Hasmy, L. Rincón, R. Hernández, V. Mujica, M. Márquez, and C. González, "On the formation of suspended noble-metal monatomic chains," Physical Review B, vol. 78, no. 11, Article ID 115409, 5 pages, 2008.

[10] A. Thiess, Y. Mokrousov, S. Blügel, and S. Heinze, "Theory and application of chain formation in break junctions," Nano Letters, vol. 8, no. 8, pp. 2144-2149, 2008.

[11] T. Shiota, A. I. Mares, A. M. C. Valkering, T. H. Oosterkamp, and J. M. Van Ruitenbeek, "Mechanical properties of Pt monatomic chains," Physical Review B, vol. 77, no. 12, Article ID 125411, 2008.

[12] T. Kizuka, "Atomic configuration and mechanical and electrical properties of stable gold wires of single-atom width," Physical Review B, vol. 77, no. 15, Article ID 155401, 2008.

[13] F. Pauly, M. Dreher, J. K. Viljas, M. Häfner, J. C. Cuevas, and P. Nielaba, "Theoretical analysis of the conductance histograms and structural properties of $\mathrm{Ag}, \mathrm{Pt}$, and $\mathrm{Ni}$ nanocontacts," Physical Review B, vol. 74, no. 23, Article ID 235106, 2006.

[14] S. López-Moreno, A. H. Romero, A. Muñoz, and U. Schwingenschlögl, "First-principles description of atomic gold chains on Ge(001)," Physical Review B, vol. 81, no. 4, Article ID 041415, 4 pages, 2010.

[15] P. C. Snijders, "Colloquium: electronic instabilities in selfassembled atom wires," Reviews of Modern Physics, vol. 82, no. 1, pp. 307-329, 2010.
[16] D. H. Wei, C. L. Gao, Kh. Zakeri, and M. Przybylski, "Pd atomic chain formation as a result of submonolayer deposition of $3 \mathrm{~d}$ metals on Pd(110)," Physical Review Letters, vol. 103, no. 22, Article ID 225504, 2009.

[17] H. Garbouj, M. Said, F. Picaud, C. Ramseyer, D. Spanjaard, and M. C. Desjonquères, "Growth of perfect and smooth Ag and Co monatomic wires on Pt vicinal surfaces: a kinetic Monte Carlo study," Surface Science, vol. 603, no. 1, pp. 22-26, 2009.

[18] C. Liu, T. Uchihashi, and T. Nakayama, "Self-alignment of Co adatoms on in atomic wires by quasi-one-dimensional electrongas-meditated interactions," Physical Review Letters, vol. 101, no. 14, Article ID 146104, 2008.

[19] K. D. Ryang, P. G. Kang, H. W. Yeom, and S. Jeong, "Structures and defects of atomic wires on $\mathrm{Si}(553)-\mathrm{Au}$ : an STM and theoretical study," Physical Review B, vol. 76, no. 20, Article ID 205325, 2007.

[20] J. Gascon, F. Kapteijn, B. Zornoza, V. Sebastián, C. Casado, and J. Coronas, "Practical approach to zeolitic membranes and coatings: state of the art, opportunities, barriers, and future perspectives," Chemistry of Materials, vol. 24, no. 15, pp. 28292844, 2012.

[21] D. A. Fedosov, A. V. Smirnov, E. E. Knyazeva, and I. I. Ivanova, "Zeolite membranes: synthesis, properties, and application," Petroleum Chemistry, vol. 51, no. 8, pp. 657-667, 2011.

[22] A. García-Suárez, Computational study of adsorption and diffusion in zeolites with cations [Ph.D. thesis], Universidad Pablo de Olavide, Seville, Spain, 2011.

[23] J. A. Thomas and A. J. H. McGaughey, "Water flow in carbon nanotubes: transition to subcontinuum transport," Physical Review Letters, vol. 102, no. 18, Article ID 184502, 2009.

[24] J. Su and H. Guo, "Effect of nanotube-length on the transport properties of single-file water molecules: transition from bidirectional to unidirectional," Journal of Chemical Physics, vol. 134, no. 24, Article ID 244513, 2011.

[25] A. Berezhkovskii and G. Hummer, "Single-file transport of water molecules through a carbon nanotube," Physical Review Letters, vol. 89, no. 6, Article ID 064503, 4 pages, 2002.

[26] H. Kumar, B. Mukherjee, S. T. Lin, C. Dasgupta, A. K. Sood, and P. K. Maiti, "Thermodynamics of water entry in hydrophobic channels of carbon nanotubes," Journal of Chemical Physics, vol. 134, no. 12, Article ID 124105, 2011.

[27] J. K. Holt, H. G. Park, W. Yinmin et al., "Fast mass transport through sub-2-nanometer carbon nanotubes," Science, vol. 312, no. 5776, pp. 1034-1037, 2006.

[28] M. Kac, G. E. Uhlenbeck, and P. C. Hemmer, "On the van der Waals theory of the vapor-liquid equilibrium. I. Discussion of a one-dimensional model," Journal of Mathematical Physics, vol. 4, no. 2, pp. 216-228, 1963.

[29] M. Kac, G. E. Uhlenbeck, and P. C. Hemmer, "On the van der waals theory of the vapor-liquid equilibrium. III. Discussion of the critical region," Journal of Mathematical Physics, vol. 5, no. 1, article 60, 10 pages, 1964.

[30] V. D. Borman, I. V. Tronin, V. N. Tronin, V. I. Troyan, B. Johansson, and N. V. Skorodumova, "Formation of high- and low-density clusters in a 1D system," Physica E, vol. 40, no. 3, pp. 643-648, 2008.

[31] V. D. Borman, V. V. Teplyakov, V. N. Tronin, I. V. Tronin, and V. I. Troyan, "Molecular transport in subnanometer channels," Journal of Experimental and Theoretical Physics, vol. 90, no. 6, pp. 950-963, 2000. 
[32] V. D. Borman, B. Johansson, N. V. Skorodumova, I. V. Tronin, V. N. Tronin, and V. I. Troyan, "Diffusion and particle mobility in 1D system," Physics Letters A, vol. 359, no. 5, pp. 504-508, 2006.

[33] V. D. Borman, V. N. Tronin, I. V. Tronin, and V. I. Troyan, "Transport of a two-component mixture in one-dimensional channels," Journal of Experimental and Theoretical Physics, vol. 98, no. 1, pp. 102-122, 2004.

[34] L. D. Landau and E. M. Lifshitz, Statistical Physics, part 1, Pergamon Press, Oxford, UK, 1980.

[35] V. N. Bogomolov, "Capillary phenomena in extremely thin zeolite channels and metal-dielectric interaction," Physical Review $B$, vol. 51, no. 23, pp. 17040-17045, 1995.

[36] S. Yu. Krylov, A. V. Prosyanov, and J. J. M. Beenakker, "One dimensional surface diffusion. II. Density dependence in a corrugated potential," Journal of Chemical Physics, vol. 107, no. 17, pp. 6970-6979, 1997.

[37] J. J. M. Beenakker, V. D. Borman, and S. Y. Krylov, "Molecular transport in the nanometer regime," Physical Review Letters, vol. 72, no. 4, pp. 514-517, 1994.

[38] Yu. N. Devyatko and V. N. Tronin, "Kinetics of the relaxation of gas-like systems to equilibrium," Journal of Experimental and Theoretical Physics, vol. 71, no. 5, pp. 880-887, 1990.

[39] V. D. Borman, S. Yu. Krylov, and A. V. Prosyanov, "Theory of nonequilibrium phenomena at a gas-solid interface," Journal of Experimental and Theoretical Physics, vol. 70, pp. 1013-1022, 1990.

[40] K. K. Sirkar, "Membrane separation technologies: current developments," Chemical Engineering Communications, vol. 157, pp. 145-184, 1997.

[41] S. T. Hwang and K. Kammermeyer, Membranes in Separations, Wiley, New York, NY, USA, 1975.

[42] J. M. Van de Graaf, "Permeation and Separation Properties of Supported Silicalite-1 Membranes," [Ph.D. thesis], University of Delft, Delft, The Netherlands, 1999.

[43] F. Kapteijn, W. J. W. Bakker, G. Zheng, J. Poppe, and J. A. Moulijn, "Permeation and separation of light hydrocarbons through a silicalite-1 membrane. Application of the generalized Maxwell-Stefan equations," The Chemical Engineering Journal and The Biochemical Engineering Journal, vol. 57, no. 2, pp. 145153, 1995.

[44] J. M. Ziman, Models of Disorder: The Theoretical Physics of Homogeneously Disordered Systems, Cambridge University Press, Cambridge, UK, 1979.

[45] I. Z. Fisher, Statistical Theory of Liquids, University of Chicago Press, Chicago, Ill, USA, 1964.

[46] R. Balescu, Equilibrium and Nonequilibrium Statistical Mechanics, Wiley, New York, NY, USA, 1975.

[47] P. Resibois and M. De Leener, Classical Kinetical Theory of Fluids, Wiley, New York, NY, USA, 1977.

[48] Y. N. Devyatko and V. N. Tronin, "Kinetics of the relaxation of gas-like systems to equilibrium," Soviet Physics. Journal of Experimental and Theoretical Physics, vol. 98, pp. 1570-1584, 1990.

[49] G. K. L. Chan and R. Finken, "Time-dependent density functional theory of classical fluids," Physical Review Letters, vol. 94, no. 18, Article ID 183001, 2005.

[50] R. E. Richards and L. V. C. Rees, "Sorption and packing of nalkane molecules in ZSM-5," Langmuir, vol. 3, no. 3, pp. 335340, 1986.

[51] W. J. W. Bakker, G. Zheng, F. Kapteijn, M. Makke, and J. A. Moulijn, Precision Process Technology, Kluwer, Amsterdam, The Netherlands, 1993.
[52] H. Van Bekkam, E. M. Flanigen, J. C. Jancen et al., Eds., Introducrion to Zeolite Science and Practice, vol. 58, Elseiver, Amsterdam, The Netherlands, 1991.

[53] L. J. P. Van den Broeke, W. J. W. Bakker, F. Kapteijn, and J. A. Moulijn, "Binary permeation through a silicalite-1 membrane," AIChE Journal, vol. 45, no. 5, pp. 976-985, 1999.

[54] G. T. Kokotailo, S. L. Lawton, D. H. Olson, and W. M. Meier, "Structure of synthetic zeolite ZSM-5," Nature, vol. 272, no. 5652, pp. 437-438, 1978.

[55] D. H. Olson, W. O. Haag, and R. M. Lago, "Chemical and physical properties of the ZSM-5 substitutional series," Journal of Catalysis, vol. 61, no. 2, pp. 390-396, 1980.

[56] R. Krishna, "A unified approach to the modelling of intraparticle diffusion in adsorption processes," Gas Separation and Purification, vol. 7, no. 2, pp. 91-104, 1993.

[57] F. Kapteijn, W. J. W. Bakker, G. Zheng, and J. A. Moulijn, "Temperature- and occupancy-dependent diffusion of nbutane through a silicalite-1 membrane," Microporous Materials, vol. 3, no. 3, pp. 227-234, 1994.

[58] W. J. W. Bakker, F. Kapteijn, J. C. Jansen, H. van Bekkum, and J. A. Moulijn, "Hoge permeabiliteit en selectiviteit bereikt. Doorbraak in ontwikkeling zeolietmembranen," Procestechnologie, vol. 3, no. 12, pp. 7-16, 1993 (Dutch).

[59] A. Tsvelik, Quantum Field Theory in Condensed Matter Physics, Cambridge, UK, 1998.

[60] H. van Beijeren, K. W. Kehr, and R. Kutner, "Diffusion in concentrated lattice gases. III. Tracer diffusion on a onedimensional lattice," Physical Review B, vol. 28, no. 10, pp. 5711$5723,1983$.

[61] G. Hummer, J. C. Rasaiah, and J. P. Noworyta, "Water conduction through the hydrophobic channel of a carbon nanotube," Nature, vol. 414, no. 6860, pp. 188-190, 2001.

[62] V. Gupta, S. S. Nivarthi, A. V. McCormick, and H. T. Davis, "Evidence for single file diffusion of ethane in the molecular sieve AlPO4-5," Chemical Physics Letters, vol. 247, no. 4-6, pp. 596-600, 1995.

[63] V. Kukla, J. Kornatowski, D. Demuth et al., "NMR studies of single-file diffusion in unidimensional channel zeolites," Science, vol. 272, no. 5262, pp. 702-704, 1996.

[64] H. Jobic, K. Hahn, J. Kärger et al., "Unidirectional and singlefile diffusion of molecules in one-dimensional channel systems. A quasi-elastic neutron scattering study," Journal of Physical Chemistry B, vol. 101, no. 30, pp. 5834-5841, 1997.

[65] S. Vasenkov and J. Kärger, "Different time regimes of tracer exchange in single-file systems," Physical Review E, vol. 66, no. 5, Article ID 052601, 4 pages, 2002.

[66] J. B. Delfau, C. Coste, C. Even, and M. Saint Jean, "Singlefile diffusion of interacting particles in a finite-sized channel," Physical Review E, vol. 82, no. 3, Article ID 031201, 2010.

[67] J.-B. Delfau, "Christophe Coste, and Michel Saint Jean. Singlefile diffusion of particles in a box: transient behaviors," Physical Review E, vol. 85, no. 6, Article ID 061111, 14 pages, 2012.

[68] P. A. Fedders, "Two-point correlation functions for a distinguishable particle hopping on a uniform one-dimensional chain," Physical Review B, vol. 17, no. 1, pp. 40-46, 1978.

[69] J. Kärger, "Straightforward derivation of the long-time limit of the mean-square displacement in one-dimensional diffusion," Physical Review A, vol. 45, no. 6, pp. 4173-4174, 1992.

[70] M. Kollmann, "Single-file diffusion of atomic and colloidal systems: asymptotic laws," Physical Review Letters, vol. 90, no. 18, Article ID 180602, 2003. 
[71] K. Hahn, J. Kärger, and V. Kukla, "Single-file diffusion observation," Physical Review Letters, vol. 76, no. 15, pp. 2762-2765, 1996.

[72] C. Rödenbeck, J. Kärger, and K. Hahn, "Exact analytical description of tracer exchange and particle conversion in single-file systems," Physical Review E, vol. 55, no. 5, pp. 5697-5712, 1997.

[73] C. Rodenbeck, J. Kärger, and K. Hahn, "Calculating exact propagators in single-file systems via the reflection principle," Physical Review E, vol. 57, no. 4, pp. 4382-4397, 1998.

[74] N. Nilius, T. M. Wallis, and W. Ho, "Localized molecular constraint on electron delocalization in a metallic chain," Physical Review Letters, vol. 90, no. 18, Article ID 186102, 4 pages, 2003.

[75] N. Agraï, A. L. Yeyatib, and M. van Ruitenbeekc, "Quantum properties of atomic-sized conductors," Physics Reports, vol. 377, no. 2-3, pp. 281-279, 2003.

[76] H. Ohnishi, Y. Kondo, and K. Takayanagi, "Quantized conductance through individual rows of suspended gold atoms," Nature, vol. 395, no. 6704, pp. 780-783, 1998.

[77] V. Rodrigues and D. Ugarte, "Real-time imaging of atomistic process in one-atom-thick metal junctions," Physical Review B, vol. 63, no. 7, Article ID 073405, 4 pages, 2001.

[78] Y. Takai, T. Kawasaki, Y. Kimura, T. Ikuta, and R. Shimizu, "Dynamic observation of an atom-sized gold wire by phase electron microscopy," Physical Review Letters, vol. 87, no. 10, Article ID 106105, 4 pages, 2001.

[79] T. Kizuka, S. Umehara, and S. Fujisawa, "Metal-insulator transition in stable one-dimensional arrangements of single gold atoms," Japanese Journal of Applied Physics, vol. 40, no. 1, pp. L71-L74, 2001.

[80] C. Untiedt, A. Yanson, R. Grande et al., "Calibration of the length of a chain of single gold atoms," Physical Review B, vol. 66, no. 8, Article ID 085418, 6 pages, 2002.

[81] N. V. Skorodumova and S. I. Simak, "Spatial configurations of monoatomic gold chains," Computational Materials Science, vol. 17, no. 2-4, pp. 178-181, 2000.

[82] N. V. Skorodumova, S. I. Simak, A. E. Kochetov, and B. Johansson, "Ab initio study of electronic and structural properties of gold nanowires with light-element impurities," Physical Review $B$, vol. 75, no. 23, Article ID 235440, 2007.

[83] N. V. Skorodumova and S. I. Simak, "Stability of gold nanowires at large Au-Au separations," Physical Review B, vol. 67, no. 12, Article ID 121404, 4 pages, 2003.

[84] V. Rodrigues, T. Fuhrer, and D. Ugarte, "Signature of atomic structure in the quantum conductance of gold nanowires," Physical Review Letters, vol. 85, no. 19, pp. 4124-4127, 2000.

[85] S. R. Bahn, N. Lopez, J. K. Nørskov, and K. W. Jacobsen, "Adsorption-induced restructuring of gold nanochains," Physical Review B, vol. 66, no. 8, Article ID 081405, 2002.

[86] S. B. Legoas, D. S. Galvão, V. Rodrigues, and D. Ugarte, "Origin of anomalously long interatomic distances in suspended gold chains," Physical Review Letters, vol. 88, no. 7, pp. 761051-761054, 2002.

[87] S. Di Napoli, A. Thiess, S. Blügel, and Y. Mokrousov, "Modeling impurity-assisted chain creation in noble-metal break junctions," Journal of Physics, vol. 24, no. 13, Article ID 135501, 2012.

[88] M. Kiguchi, R. Stadler, I. S. Kristensen, D. Djukic, and J. M. van Ruitenbeek, "Evidence for a single hydrogen molecule connected by an atomic chain," Physical Review Letters, vol. 98, no. 14, Article ID 146802, 2007.

[89] C. Q. Sun, C. M. Li, S. Li, and B. K. Tay, "Breaking limit of atomic distance in an impurity-free monatomic chain," Physical Review B, vol. 69, no. 24, Article ID 245402, 2004.
[90] C. Kittel, Introduction to Solid State Physics, Wiley, New-York, NY, USA, 1995.

[91] T. Hill, Statistical Mechanics, McGraw-Hill Book Company, New York, NY, USA, 1956.

[92] E. M. Lifshitz and L. P. Pitaevskii, Statistical Physics, part 2, Pergamon Press, Oxford, UK, 1980.

[93] A. Calzolari, C. Cavazzoni, and M. B. Nardelli, "Electronic and transport properties of artificial gold chains," Physical Review Letters, vol. 93, no. 9, pp. 1-96404, 2004.

[94] G. M. Francis, L. Kuipers, J. R. A. Cleaver, and R. E. Palmer, "Diffusion controlled growth of metallic nanoclusters at selected surface sites," Journal of Applied Physics, vol. 79, no. 6, pp. 29422947, 1996.

[95] E. Y. Zarechnaya, N. V. Skorodumova, S. I. Simak, B. Johansson, and E. I. Isaev, "Theoretical study of linear monoatomic nanowires, dimer and bulk of $\mathrm{Cu}, \mathrm{Ag}, \mathrm{Au}, \mathrm{Ni}, \mathrm{Pd}$ and $\mathrm{Pt}$," Computational Materials Science, vol. 43, no. 3, pp. 522-530, 2008.

[96] L. D. Landau, L. P. Pitaevskii, E. M. Lifshitz, and A. M. Kosevich, Course of Theoretical Physics, vol. 7 of Theory of Elasticity, Butterworth-Heinemann, 3 edition, 1986.

[97] J. M. Van de Graaf, F. Kapteijn, and J. A. Moulijn, "Modeling permeation of binary mixtures through zeolite membranes," AIChE Journal, vol. 45, no. 3, pp. 497-511, 1999.

[98] Yu. L. Klimontovich, Statistical Physics, Harwood Academic, New-York, NY, USA, 1986.

[99] D. N. Voskresenskii, Multiparticle Effects in Solids, Energoatomizdat, Moscow, Russia, 1983.

[100] O. G. Bakunin, "Mysteries of diffusion and labyrinths of destiny," Physics-Uspekhi, vol. 46, no. 3, pp. 309-313, 2003.

[101] G. N. Sarkisov, "Approximate equations of the theory of liquids in the statistical thermodynamics of classical liquid systems," Physics-Uspekhi, vol. 42, no. 6, pp. 545-561, 1999.

[102] F. Baletto and R. Ferrando, "Structural properties of nanoclusters: energetic, thermodynamic, and kinetic effects," Reviews of Modern Physics, vol. 77, no. 1, pp. 371-423, 2005. 

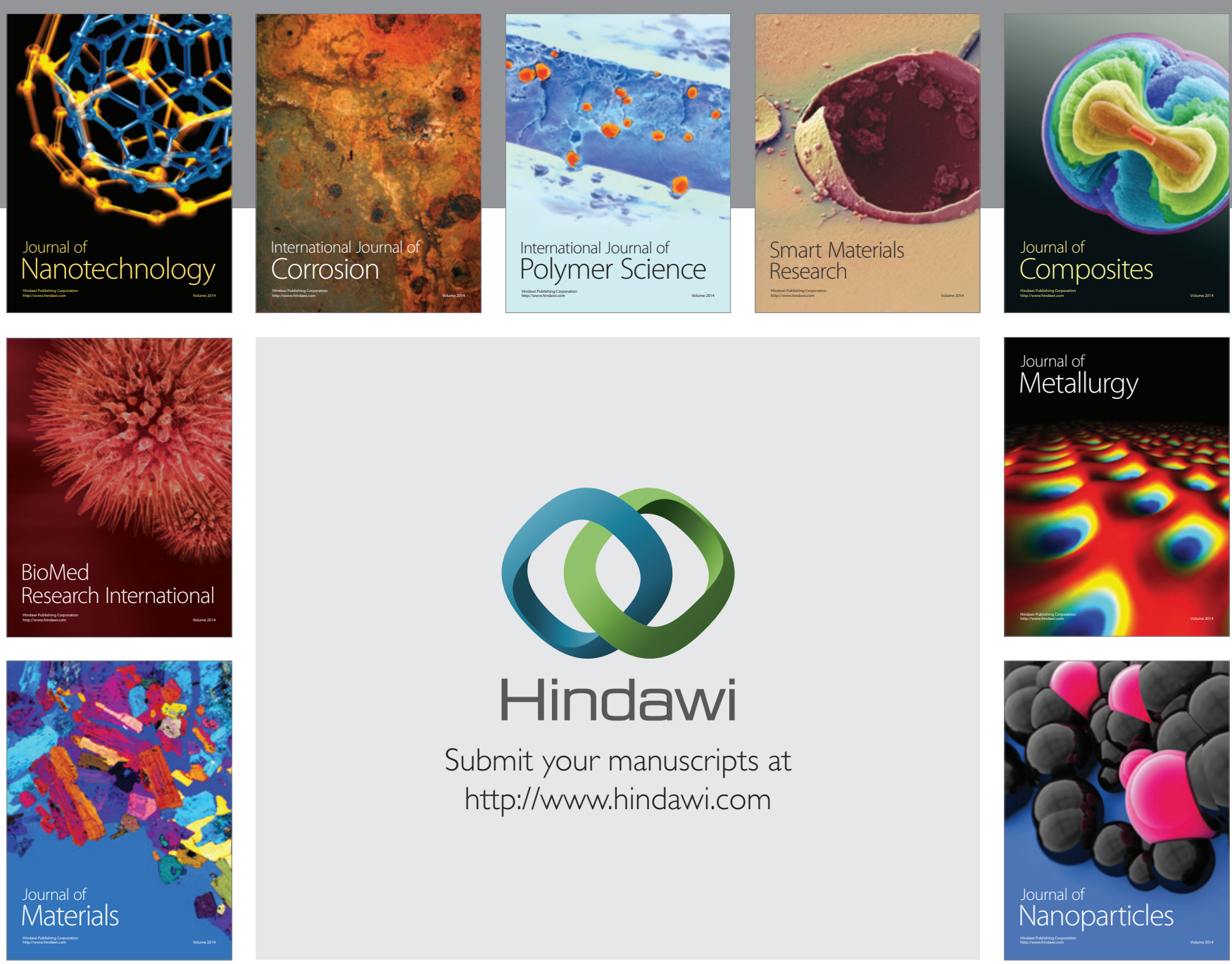

Submit your manuscripts at http://www.hindawi.com
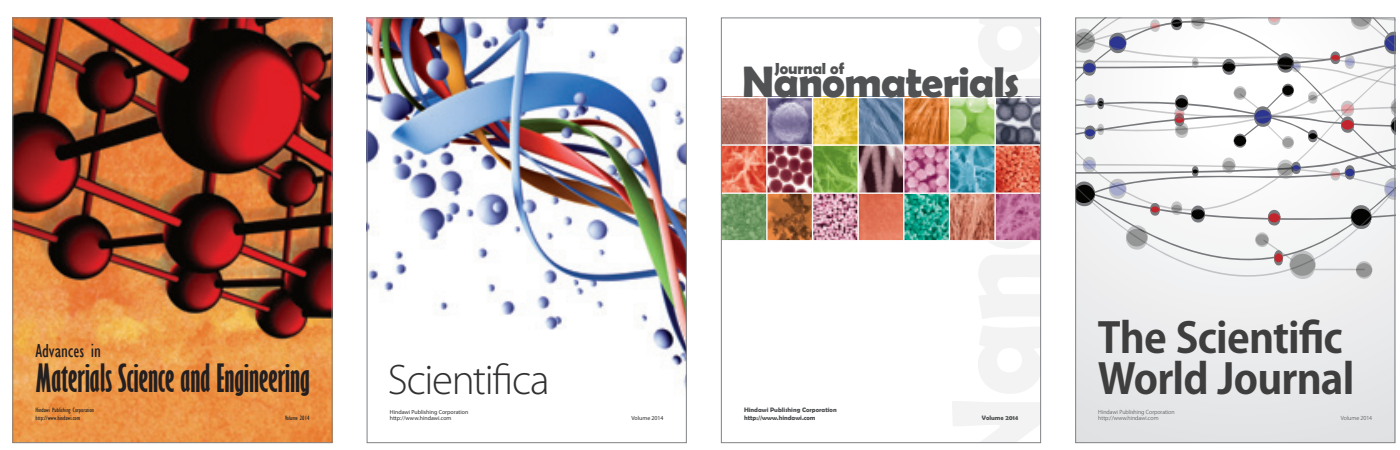

\section{The Scientific World Journal}
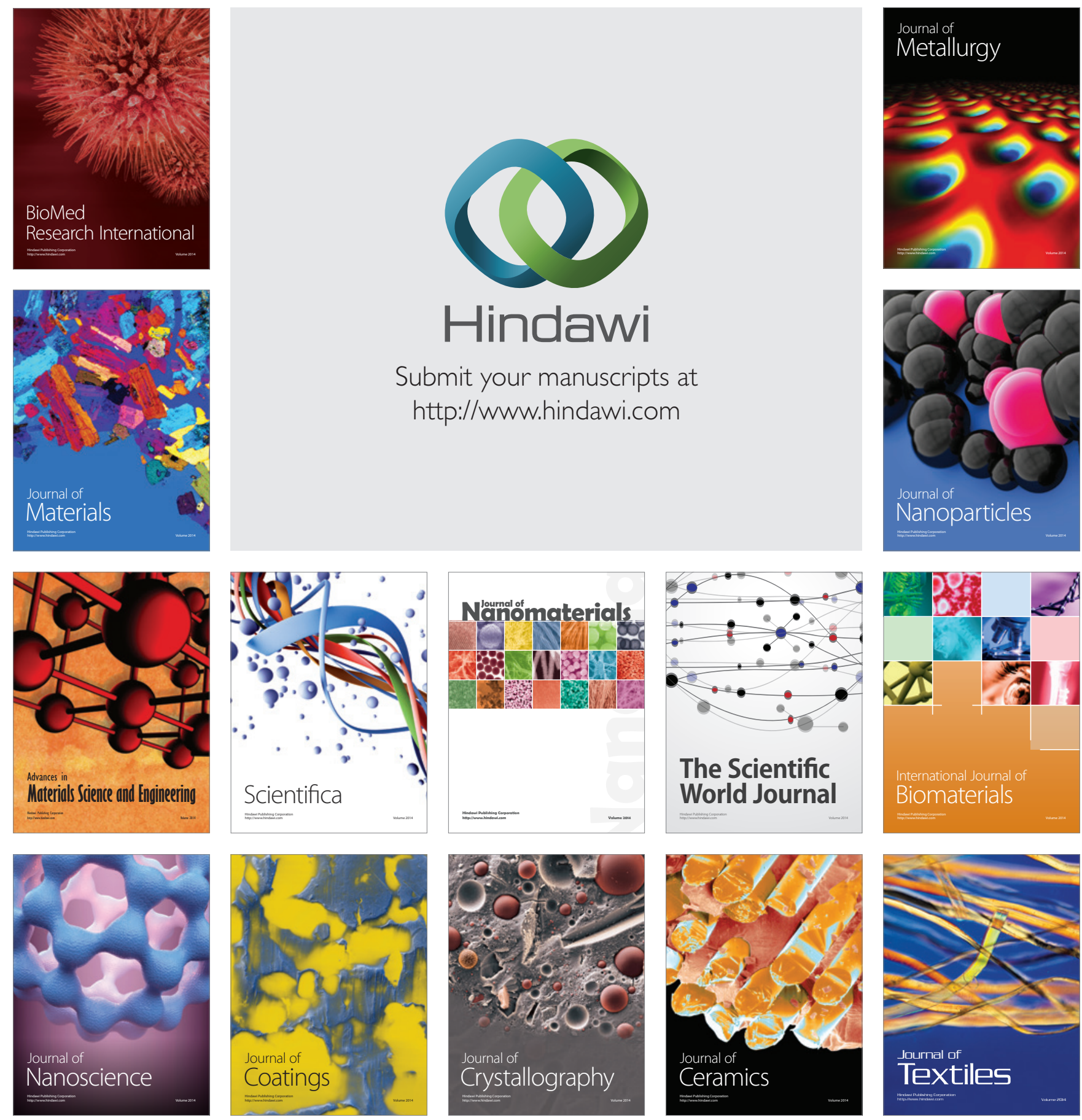